\title{
Mathematical Apparatus for Selection of Optimal Parameters of Technical, Technological Systems and Materials Based on Vector Optimization
}

\author{
Yu Mashunin \\ Far Eastern Federal University, Vladivostok, Russia \\ Email: mashunin@mail.ru
}

How to cite this paper: Mashunin, Y. (2020) Mathematical Apparatus for Selection of Optimal Parameters of Technical, Technological Systems and Materials Based on Vector Optimization. American Journal of Operations Research, 10, 173-239. https://doi.org/10.4236/ajor.2020.105013

Received: June 4, 2020

Accepted: September 24, 2020

Published: September 27, 2020

Copyright ( 2020 by author(s) and Scientific Research Publishing Inc. This work is licensed under the Creative Commons Attribution International License (CC BY 4.0).

http://creativecommons.org/licenses/by/4.0/

(c) (i) Open Access

\begin{abstract}
We presented Mathematical apparatus of the choice of optimum parameters of technical, technological systems and materials on the basis of vector optimization. We have considered the formulation and solution of three types of tasks presented below. First, the problem of selecting the optimal parameters of technical systems depending on the functional characteristics of the system. Secondly, the problem of selecting the optimal parameters of the process depending on the technological characteristics of the process. Third, the problem of choosing the optimal structure of the material depending on the functional characteristics of this material. The statement of all problems is made in the form of vector problems of mathematical (nonlinear) programming. The theory and the principle of optimality of the solution of vector tasks it is explained in work of https://rdcu.be/bhZ8i. The implementation of the methodology is shown on a numerical example of the choice of optimum parameters of the technical, technological systems and materials. On the basis of mathematical methods of solution of vector problems we developed the software in the MATLAB system. The numerical example includes: input data (requirement specification) for modeling; transformation of mathematical models with uncertainty to the model under certainty; acceptance of an optimal solution with equivalent criteria (the solution of numerical model); acceptance of an optimal solution with the given priority of criterion.
\end{abstract}

\section{Keywords}

Vector Optimization, Methods of Solution of Vector Problems,

Modeling of a Technical System, Modeling Operation of

Technological Processes, Modeling of Structure of Material 


\section{Introduction}

The problem of high quality production is associated with the creation of technical, answering to the modern achievements of science and technology. The functioning of technical, technological systems, as well as the structure of materials depend on a set of functional characteristics that must be considered at the design stage. Improvement of one of the characteristics leads to deterioration of other characteristics. And it is necessary for improvement of quality of the produced product improvements of all characteristics in total. For the solution of such problems we use the theory and methods of vector (multi-criteria) optimization [1]-[16]. Development of the theory and methods of vector optimization was carried out both in Russia [1]-[17], and in other countries [18]-[24].

The purpose of this work is to create a methodology for selecting the optimal parameters of technical, technological systems and materials based on vector optimization. The methodology includes the construction of a mathematical model for an object or decision-making system, an algorithm and software for solving a vector problem of mathematical programming.

For realization of a goal in work the following problems are considered and solved. The article presents the construction of a mathematical model as a vector problem of mathematical programming for three types of optimal decision-making problems. The first task is related to the choice of optimal parameters of technical systems, which depend on a set of functional characteristics of the system. The study of this class of problems is presented in [2]-[10] [12]. The second task is bound the choice of optimum parameters of the technological process depending on running characteristics on this process [11] [17]. The third problem is the problem of choosing the optimal structure (components) of the material depending on the functional characteristics of this material [2] [13]. It is assumed that all three tasks are used at the design stage and creation of a new technical object (system) within the framework of information and mathematical concepts [14] [15]. The implementation of the methodology is presented on the solution of numerical problems of decision-making in the engineering systems by two, four parameters. It is assumed that all three tasks are used at the design stage and creation of a new technical object (system) within the framework of information and mathematical concepts [14] [15]. The implementation of the methodology is presented on the solution of numerical problems of decision-making in a technical system by four parameters.

\section{Setting the Problem of Selection of Optimal Parameters of Engineering Systems}

\subsection{Building a Mathematical Model of a Technical System in the Form of a Vector Optimization Problem}

Please As an "object for optimal decision-making" we use "technical system". Statement of a problem is executed according to [5] [8] [9] [15].

The technical system which functioning depends on $\mathbf{N}$-a set of design data is considered: 
$X=\left\{x_{1} x_{2} \cdots x_{N}\right\}, N$-number of parameters, each of which lies in the set limits:

$$
x_{j}^{\min } \leq x_{j} \leq x_{j}^{\max }, j=\overline{1, N}
$$

or

$$
X^{\min } \leq X \leq X^{\max },
$$

where $x_{j}^{\min }, x_{j}^{\max }, \forall j \in N$-lower and top limits of change of the vector of parameters of the technical system.

The result of functioning of the technical system is defined by a set $K$ to technical characteristics of $f_{k}(X), k=\overline{1, K}$, which functionally depend on design data $X=\left\{x_{j}, j=\overline{1, N}\right\}$, in total they represent a vector function:

$$
F(X)=\left(f_{1}(X) f_{2}(X) \cdots f_{K}(X)\right)^{\mathrm{T}} .
$$

The set of characteristics (criteria) to is subdivided into two subsets $K_{1}$ and $K_{2}$ : $\boldsymbol{K}=\boldsymbol{K}_{1} \cup \boldsymbol{K}_{2}$ is a subset of technical characteristics, the numerical values of which are desirable to obtain as high as possible: $f_{k} \rightarrow \max , k=\overline{1, K_{1}}$.

$K_{2}$-it subsets of technical characteristics which numerical sizes it is desirable to receive as it is possible below: $f_{k} \rightarrow \min , k=\overline{1, K_{1}+1}, K_{2} \equiv \overline{K_{1}+1, K}$. The mathematical model should, firstly, the purposes of the technical system which are presented by the characteristics of $F(X)$, secondly, to consider $X^{\min } \leq X \leq X^{\max }$ restrictions. The mathematical model of the technical system which solves in general a problem of a choice of the optimum design decision (a choice of optimum parameters), we will present in the form of a vector problem of mathematical programming.

$$
\begin{gathered}
\text { Opt } F(X)=\left\{\max F_{1}(X)=\left\{\max f_{k}(X), k=\overline{1, K_{1}}\right\},\right. \\
\left.\min F_{2}(X)=\left\{\min f_{k}(X), k=\overline{1, K_{2}}\right\}\right\}, \\
G(X) \leq 0, \\
x_{j}^{\min } \leq x_{j} \leq x_{j}^{\max }, j=\overline{1, N},
\end{gathered}
$$

where $X$ is the vector of controlled variables (constructive parameters) from (1); $F(X)=\left\{f_{k}, k=\overline{1, K}\right\}$-vector criterion which everyone a component submits the characteristic of the technical system (1a)-(2a) which is functionally depending on the vector of variables $X$; in (3a) $G(X)=\left\{g_{1}(X) g_{2}(X) \cdots g_{M}(X)\right\}^{\mathrm{T}}$-vector-a function of the restrictions imposed on functioning of the technical system, $M$ - a set of restrictions.

Restrictions are defined proceeding in them technological, physical and to that similar processes and can be presented by functional restrictions, for example:

$$
f_{k}^{\min } \leq f_{k}(X) \leq f_{k}^{\max }, k=\overline{1, K} .
$$

It is supposed that the $f_{k}, k=\overline{1, K}$ functions are differentiated and convex, $g_{i}(X), i=\overline{1, M}$, are continuous, and (3a)-(4a) set of admissible points of $\mathcal{S}$ set 
by restrictions isn't empty and represents a compact:

$$
\boldsymbol{S}=\left\{X \in \boldsymbol{R}^{n} \mid G(X) \leq 0, X^{\min } \leq X \leq X^{\max }\right\} \neq \varnothing .
$$

Criteria and restrictions (1a)-(4a) form mathematical model of technical system. It is required to find such vector of the $X^{o} \in S$ parameters at which everyone a component the vector-functions

$$
F_{1}(X)=\left\{f_{k}(X), k=\overline{1, K_{1}}\right\}
$$

accepts the greatest possible value, and a vector-functions

$$
F_{2}(X)=\left\{f_{k}(X), k=\overline{1, K_{2}}\right\}
$$

are accepted by the minimum value.

To a substantial class of technical systems which can be presented by a vector problem (1a)-(4a), it is possible to refer their rather large number of tasks from various branches of economy of the state: as electro engineering [6], airspace [17] [18], metallurgical (choice of optimal structure of material), chemical [20], etc.

In this article, the technical system is considered in statics. But technical systems can be considered in dynamics [6], using differential-difference methods of transformation, conducting research for a small discrete period $\Delta t \in T$.

\subsection{Building a Mathematical Process Model in the Form of a Vector Optimization Problem}

Please As an object of a research we use "technological process". The problem of decision-making in technology in the production of products is formulated in accordance with the works [11] [25].

We consider a technological process (e.g., Hybrid laser arc welding [25], in which ze41-T5 alloy was chosen as the material to be welded with AZ61 alloy as the filler material). Activity of technological process depends on a particular set of conditions-design data: $X=\left\{x_{1} x_{2} \cdots x_{N}\right\}$, (for example: laser powers; speeds of movement; feed rates of a wire; current; frequencies) we Will designate [25]. Let's denote $\boldsymbol{N}$-set of constructive parameters. Each parameter of the technological process lies in the given limits:

$$
x_{j}^{\min } \leq x_{j} \leq x_{j}^{\max }, j=\overline{1, N} \text {, or } X^{\min } \leq X \leq X^{\max },
$$

where $x_{j}^{\min }, x_{j}^{\max }, \forall j \in N$-the lower and top limits of change of a vector of parameters of technological process, $N$ is the number of parameters.

The result of functioning is defined by a set of running characteristics $f_{k}(X), k=\overline{1, K}$, which functionally depend on design data of technological process of $X=\left\{x_{j}, j=\overline{1, N}\right\}$, (for example: the depth of the weld; underfill; percentage defect; total accumulated pore length). In total all running characteristics represent a vector function:

$$
F(X)=\left(f_{1}(X) f_{2}(X) \cdots f_{K}(X)\right)^{\mathrm{T}},
$$

where $\boldsymbol{K}$-a set (number) of technological characteristics (criteria). 
The set of characteristics $K$ is subdivided into two subsets of $\boldsymbol{K}_{1}$ and $\boldsymbol{K}_{2}$ : $\boldsymbol{K}=\boldsymbol{K}_{1} \cup \boldsymbol{K}_{2}$ is a subset of technological characteristics, the numerical values of which are desirable to obtain as high as possible: $f_{k} \rightarrow \max , k=\overline{1, K_{1}} . K_{2}$ are subsets of technological characteristics, the numerical values of which are desirable to obtain as low as possible: $f_{k} \rightarrow \min , k=\overline{1, K_{1}+1}, K_{2} \equiv \overline{K_{1}+1, K}$.

Mathematical model has to reflect, first, the purposes of the technological process which are presented by the characteristics of $F(X)$, and secondly, to take into account the constraints of $X^{\min } \leq X \leq X^{\max }$. The mathematical model of the technological process solving in general a problem of the choice of optimum parameters of the technological process can be presented in the form of a vector problem of mathematical programming.

$$
\begin{gathered}
\text { Opt } F(X)=\left\{\max F_{1}(X)=\left\{\max f_{k}(X), k=\overline{1, K_{1}}\right\},\right. \\
\left.\min F_{2}(X)=\left\{\min f_{k}(X), k=\overline{1, K_{2}}\right\}\right\}, \\
G(X) \leq 0, \\
x_{j}^{\min } \leq x_{j} \leq x_{j}^{\max }, j=\overline{1, N},
\end{gathered}
$$

where $X$ is the vector of operated variable (design parameters) of the technological process; $F(X)=\left\{f_{k}, k=\overline{1, K}\right\}$-vector criterion which each component represents the characteristic of the technical system (1b)-(2b) which is functionally depending on a vector of variables $X$; in (3b) $G(X)=\left\{g_{1}(X) g_{2}(X) \cdots g_{M}(X)\right\}^{\mathrm{T}}$ is a vector function of the restrictions imposed on functioning of the technological process, $M$ is a set of constraints. The constraints are defined flowing past in them technological, physical and to that similar processes and can be presented by the functional restrictions, for example:

$$
f_{k}^{\min } \leq f_{k}(X) \leq f_{k}^{\max }, k=\overline{1, K} .
$$

It is supposed that the functions $f_{k}(X), k=\overline{1, K}$ are differentiated and convex, $g_{i}(X), i=\overline{1, M}$ are continuous, and $\boldsymbol{S}=\left\{X \in \boldsymbol{R}^{N} \mid G(X) \leq 0, X^{\min } \leq X \leq X^{\max }\right\} \neq \varnothing$.

The (1b)-(4b) ratios form mathematical model of the technological process. It is required to find such vector of the $X^{0} \in S$ parameters at which each component of a vector function of $F_{1}(X)=\left\{f_{k}(X), k=\overline{1, K_{1}}\right\}$ accepts the greatest possible value, and vector functions of $F_{2}(X)=\left\{f_{k}(X), k=\overline{1, K_{2}}\right\}$ accepts minimum value.

\subsection{Building a Mathematical Model of a Material Structure in the Form of a Vector Optimization Problem}

The structure of the material is characterized by a particular set of the functional characteristics which include mechanical and physical and chemical properties of the material. One group of properties (the functional characteristics) of the material is characterized by the fact that it is desirable to receive them on the numerical value as much as possible (for example, strength), other group of 
properties is characterized by the fact that is desirable to receive them on the numerical value less as. As a rule, improvement on one of these characteristics leads to a deterioration of the other. In General, it is required to choose such a composition of the material that all the properties of the material were as good as possible in the aggregate. Chemical composition of material is determined by the percentage (per unit volume) of a multiple component material, which in the amount equal to one hundred percent.

The composition of material of any product, technical system depends on $V$-a set of components of material: $Y=\left\{\begin{array}{llll}y_{1} & y_{2} & \cdots & y_{V}\end{array}\right\}$, other record of a vector $Y=\left\{y_{v}, v=\overline{1, V}\right\}, V$ is the number of components of which it can be made (is made) material, $y_{v}$-size as a percentage $v$-th of a material component, each of which lies in the given limits:

$$
y_{v}^{\min } \leq y_{v} \leq y_{v}^{\max }, v=\overline{1, V} \text {, or } Y^{\min } \leq Y \leq Y^{\max },
$$

where $y_{v}^{\min }, y_{v}^{\max }, \forall v \in V$ - the lower and top limits of change of a vector of the material of components; $Y=\left\{y_{v}, v=\overline{1, V}\right\}$ is the vector of unknowns defining the complete set and size of all components which are a part of material; $\sum_{v \in V} y_{v}=100 \%$ - the sum of all components of the material is equal to hundred percent.

The composition of the material is estimated by a set $H$ of physical properties (characteristics) of the material: $h_{k}(Y), k=\overline{1, K}$ which functionally depend on the design parameters (components) of $Y, k$-the index of a type of physical properties of the material, $k=\overline{1, K}$ where $K$-the number of types of properties (the functional characteristics) of the material, we will present them in the form a vector-functions:

$$
H(Y)=\left(h_{1}(Y) h_{2}(Y) \cdots h_{K}(Y)\right)^{\mathrm{T}} \text { or } H(Y)=\left\{h_{k}(Y), k=\overline{1, K}\right\}^{\mathrm{T}}
$$

The set of the functional characteristics of the material $K$ is subdivided into two subsets of $K_{1}$ and $K_{2}: \quad K=K_{1} \cup K_{2} . K_{1}$ is a subset of characteristics of material which numerical values it are desirable to receive as it is possible above: $h_{k}(Y) \rightarrow \max , k=\overline{1, K_{1}}$.

$K_{2}$ are subsets of characteristics of material which numerical values it is desirable to receive, as low as possible: $h_{k}(Y) \rightarrow \min , k=\overline{1, K_{1}+1}, K_{2} \equiv \overline{K_{1}+1, K}$.

Using the functional characteristics of material $H(Y)=\left\{h_{k}(Y), k=\overline{1, K}\right\}^{\mathrm{T}}$ as criteria, and parametrical restrictions $Y^{\min } \leq Y \leq Y^{\max }$ as the change limits imposed on each type of components, the mathematical model of the material solving in general a problem of the choice of optimum structure of the material (the optimal design solution), we will present a vector problem of mathematical programming:

$$
\begin{gathered}
\text { Opt } F(X)=\left\{\max F_{1}(X)=\left\{\max h_{k}(X), k=\overline{1, K_{1}}\right\},\right. \\
\left.\min F_{2}(X)=\left\{\min h_{k}(X), k=\overline{1, K_{2}}\right\}\right\},
\end{gathered}
$$




$$
\begin{gathered}
G(X) \leq 0, \sum_{v \in V} y_{v}=100, \\
y_{v}^{\min } \leq y_{v} \leq y_{v}^{\max }, v=\overline{1, V},
\end{gathered}
$$

is the vector of the operated variables (a material component) from $Y^{\min } \leq Y \leq Y^{\max }, V$-number of components; $H(Y)=\left\{h_{k}(Y), k=\overline{1, K}\right\}^{\mathrm{T}}$-vector criterion which each function submits the characteristic (property) of the material which is functionally depending on a vector of variables $Y$; in (3c) $G(X)=\left\{g_{1}(Y) g_{2}(Y) \cdots g_{M}(Y)\right\}^{\mathrm{T}}, \sum_{v \in V} y_{v}=100$ is a vector function of the restrictions imposed on structure of the material, $M$ is a set of restrictions. Restrictions are defined technological, physical and similar properties, processes (technologies) and can be presented by the functional restrictions, for example, $h_{k}^{\min } \leq h_{k}(Y) \leq h_{k}^{\max }, k=\overline{1, K}$. It is supposed that the functions $h_{k}(Y), k=\overline{1, K}$ are differentiated and convex, $g_{i}(Y), i=\overline{1, M}$ are continuous, and (3c)-(4c) set of admissible points of $\boldsymbol{S}$ set by restrictions are not empty and represents a compact:

$$
\boldsymbol{S}=\left\{Y \in \boldsymbol{R}^{n} \mid G(X) \leq 0, Y^{\min } \leq X \leq Y^{\max }\right\} \neq \varnothing .
$$

Ratios (1c)-(4c) form mathematical model of the material. It is required to find such vector of the $Y^{o} \in S$ parameters at which each component (characteristic) the vector-the $H_{1}(Y)=\left\{h_{k}(Y), k=\overline{1, K_{1}}\right\}$ functions accepts the greatest possible value, and a vector-the $H_{2}(Y)=\left\{h_{k}(Y), k=\overline{1, K_{2}}\right\}$ functions accepts minimum value.

In total the mathematical model of the material (1c)-(4c) can be interpreted as systems approach to the study of the material.

\section{Problem of Vector Optimization as Mathematical Model of a Technical System, Technological Process and Structure of Material with Conditions of Certainty and Uncertainty}

\subsection{A Vector Problem of Mathematical Programming with Conditions of Certainty and Uncertainty}

The Mathematical models of a technical system (1a)-(4a), technological process (1b)-(4b) and structures of material (1c)-(4c) are constructed in the assumption that the functional dependence of each criterion (characteristic) and restrictions from parameters of the studied object is known. In real life such functional dependence of criterion from parameters extremely infrequent, i.e. there are conditions of uncertainty. We present a vector optimization problem with certainty and uncertainty conditions.

The conditions of a certainty are characterized by the fact that the functional dependence of each characteristic and restrictions on the parameters of the studied object is known [2] [5] [7] [9].

The conditions of uncertainty are characterized by the fact that the initial data characterizing the studied object are presented: a) random, b) fuzzy, or, c) incomplete data. Therefore, we lack sufficient information about the functional dependence of each characteristic and restrictions on the parameters [5] [10] 
[14]. For options a) and b) basic data have to be transformed to option c) and are presented in the table form. In work the option c) - with not full data which are, as a rule, obtained from experimental data is investigated.

In real life, the conditions of certainty and uncertainty are combined. The process model should also reflect these conditions. We will present a model of the technological process under certainty and uncertainty in the aggregate:

$$
\begin{aligned}
& \text { Opt } F(X)=\left\{\max F_{1}(X)=\left\{\max f_{k}(X), k=\overline{1, K_{1}^{\text {def }}}\right\},\right. \\
& \max I_{1}(X) \equiv\left\{\max f_{k}\left(X_{i}, i=\overline{1, M}\right)\right\}^{\mathrm{T}}, k=\overline{1, K_{1}^{\text {unc }},} \\
& \min F_{2}(X)=\left\{\min _{k}(X), k=\overline{1, K_{2}^{\text {def }}},\right. \\
&\left.\left.\min I_{2}(X) \equiv\left\{\min f_{k}\left(X_{i}, i=\overline{1, M}\right)\right\}^{\mathrm{T}}, k=\overline{1, K_{2}^{\text {unc }}}\right\}\right\}, \\
& \text { at } \text { restriction } f_{k}^{\min } \leq f_{k}(X) \leq f_{k}^{\max }, k=\overline{1, K}, \\
& x_{j}^{\min } \leq x_{j} \leq x_{j}^{\max }, j=\overline{1, N},
\end{aligned}
$$

where $X=\left\{x_{j}, j=\overline{1, N}\right\}$ is the vector of controlled variables (input parameters of the studied object); $F(X)=\left\{F_{1}(X) F_{2}(X) I_{1}(X) I_{2}(X)\right\}$ is a vector criterion, each component of which represents a vector of criteria (output characteristics of the studied object). The magnitude of the characteristic (function) depends on the discrete values of the vector of variables $X . F_{1}(X), F_{2}(X)$ is the set of functions max and min, respectively; $I_{1}(X), I_{2}(X)$ are the set of discrete values of the characteristics max and min, respectively; $\overline{1, K_{1}^{\text {def }}}, \overline{1, K_{2}^{\text {def }}}$, (definiteness), $\overline{1, K_{1}^{\text {unc }}}, \overline{1, K_{2}^{\text {unc }}}$, (uncertainty) a set of criteria max and min formed under certainty and uncertainty; in (3), $f_{k}^{\min } \leq f_{k}(X) \leq f_{k}^{\max }, k=\overline{1, K}$ is a vector-function of restrictions imposed on the operation of the technological process, $x_{j}^{\min } \leq x_{j} \leq x_{j}^{\max }, j=\overline{1, N}$ are parametric restrictions imposed on the studied object.

\subsection{Transformation of a Problem of Decision-Making in the Conditions of Uncertainty into a Problem of Vector Optimization in the Conditions of Certainty}

Elimination of uncertainty consists in the use of qualitative and quantitative descriptions of the object under study which can be received, for example, on the principle of "input-output". Transformation of such information-initial data into functional dependence is carried out by using mathematical methods (the regression analysis) [7] [10] [13].

The transformation of the vector function (criteria) is carried out by the method of least squares, $\min \sum_{i=1}^{M}\left(y_{i}-\overline{y_{i}}\right)^{2}$, where by $y_{i}, i=\overline{1, M}$-experimental data; $\overline{y_{i}}, i=\overline{1, M}$, their sizes received for one-factorial model by means of function $\bar{y}_{i}=f\left(X_{i}, A\right), X_{i}=\left\{x_{i}\right\}$. The function $f\left(X_{i}, A\right)$ we present in form of polynomial. In the applied part of the polynomial of the second degree is used.

As a result of this transformation, the source data in (1) and (2): 


$$
\left\{f_{k}\left(X_{i}, i=\overline{1, M}\right)\right\}^{\mathrm{T}}, k=\overline{1, K_{1}^{\text {unc }}},\left\{f_{k}\left(X_{i}, i=\overline{1, M}\right)\right\}^{\mathrm{T}}, k=\overline{1, K_{2}^{\text {unc }}}
$$

in problems of decision-making in the conditions of uncertainty the functions: $f_{k}(X), k=\overline{1, K_{1}^{u n c}}, f_{k}(X), k=\overline{1, K_{2}^{u n c}}$ are transformed.

As a result, a vector problem with conditions of certainty and uncertainty (1)-(4) is transformed into a vector problem under conditions of certainty:

$$
\begin{gathered}
\text { Opt } F(X)=\left\{\max F_{1}(X)=\left\{\max f_{k}(X), k=\overline{1, K_{1}}\right\},\right. \\
\left.\min F_{2}(X)=\left\{\min f_{k}(X) . k=\overline{1, K_{2}}\right\}\right\}, \\
\text { at restriction } f_{k}^{\min } \leq f_{k}(X) \leq f_{k}^{\max }, k=\overline{1, K}, \\
x_{j}^{\min } \leq x_{j} \leq x_{j}^{\max }, j=\overline{1, N},
\end{gathered}
$$

where $F(X)=\left\{f_{k}, k=\overline{1, K}\right\}$ is a vector criterion, each component of which represents a characteristic of the object under study, functionally dependent on the vector of variables $X$; a subset of the criteria:

$$
K_{1}=K_{1}^{\text {def }} \cup K_{1}^{u n c}, K_{2}=K_{2}^{\text {def }} \cup K_{2}^{\text {unc }}[12] \text { [13]. }
$$

The vector mathematical programming problem (5)-(8) is analogous to mathematical models (1a)-(4a), (1b)-(4b), (1c)-(4c).

\section{Mathematical Modeling Apparatus: Theory and Methods of Vector Optimization}

The theory of vector optimization includes theoretical foundations (axiomatic) and methods of the solution of vector problems with equivalent criteria and with the given criterion priority. The theory is a basis of mathematical apparatus of modeling of "object for optimal decision-making" which allows you to select any point from a set of points, optimum across Pareto and to show why she is optimum. We presented axiomatic and methods for solving vector optimization problems (5)-(8) with equivalent criteria [1] [16].

\subsection{The Axioms and the Principle of Optimality for Vector Optimization with the Equivalent Criteria}

Definition 1. (Definition of the relative assessment of the criterion).

In a vector problem (5)-(8) we will enter designation:

$\lambda_{k}(X)=\frac{f_{k}(X)-f_{k}^{0}}{f_{k}^{*}-f_{k}^{0}}, \forall k \in K$ is the relative estimate of a point $X \in S \quad k$ th criterion; $f_{k}(X)-k$ th criterion at the point $X \in S ; f_{k}^{*}$-value of the $k$ th criterion at the point of optimum $X_{k}^{*}$, obtained in vector problem (5)-(8) of individual $k$ th criterion; $f_{k}^{0}$ is the worst value of the $k$ th criterion (ant optimum) at the point $X_{k}^{0}$ (Superscript 0-zero) on the admissible set $S$ in vector problem (5)-(8); the task at $\max (5),(7),(8)$ the value of $f_{k}^{0}$ is the lowest value of the $k$ th criterion

$f_{k}^{0}=\min _{X \in S} f_{k}(X) \quad \forall k \in K_{1}$ and taskmin $f_{k}^{0}$ is the greatest:

$f_{k}^{0}=\max _{X \in S} f_{k}(X) \quad \forall k \in K_{2}$. 
The relative estimate of the $\lambda_{k}(X), \forall k \in K$ is first, measured in relative units; secondly, the relative assessment of the $\lambda_{k}(X), \forall k \in K$ : on the admissible set is changed from zero in a point of $X_{k}^{0}$

$$
\forall k \in K \lim _{X \rightarrow X_{k}^{0}} \lambda_{k}(X)=0,
$$

to the unit at the point of an optimum of $X_{k}^{*}: \forall k \in K \quad \lim _{X \rightarrow x_{k}^{*}} \lambda_{k}(X)=1$ :

$$
\forall k \in K \quad 0 \leq \lambda_{k}(X) \leq 1, \quad X \in S .
$$

this allows the comparison criteria, measured in relative units, among themselves by joint optimization.

Axiom 1. (About equality and equivalence of criteria in an admissible point of vector problems) In of vector problems of mathematical programming two criteria with the indexes $k \in \boldsymbol{K}, q \in \boldsymbol{K}$ shall be considered as equal in $X \in \boldsymbol{S}$ point if relative estimates on $k$ th and $q$ th to criterion are equal among themselves in this point, i.e. $\lambda_{k}(X)=\lambda_{q}(X), k, q \in \boldsymbol{K}$. We will consider criteria equivalent in vector problems of mathematical programming if in, $X \in S$ point when comparing in the numerical size of relative estimates of $\lambda_{k}(X), k=\overline{1, K}$, among themselves, on each criterion of $f_{k}(X), k=\overline{1, K}$, and, respectively, relative estimates of $\lambda_{k}(X)$, isn't imposed conditions about priorities of criteria.

Definition 2. (Definition of a minimum level among all relative estimates of criteria).

The relative level $\lambda$ in a vector problem represents the lower assessment of a point of $X \in S$ among all relative estimates of $\lambda_{k}(X), k=\overline{1, K}$ :

$$
\forall X \in S \quad \lambda \leq \lambda_{k}(X), k=\overline{1, K},
$$

the lower level for performance of a condition (5) in an admissible point of $X \in S$ is defined by a formula

$$
\forall X \in S \quad \lambda=\min _{k \in K} \lambda_{k}(X) .
$$

Ratios (9) and (10) are interconnected. They serve as transition from operation (6) of definition of min to restrictions (9) and vice versa.

The level $\lambda$ allows to unite all criteria in a vector problem one numerical characteristic of $\lambda$ and to make over her certain operations, thereby, carrying out these operations over all criteria measured in relative units. The level $\lambda$ functionally depends on the $X \in S$ variable, changing $X$, we can change the lower level $-\lambda$. From here we will formulate the rule of search of the optimum decision.

Definition 3. (The principle of an optimality with equivalent criteria).

The vector problem of mathematical programming at equivalent criteria is solved, if the point of $X^{0} \in S$ and a maximum level of $\lambda^{\circ}$ (the top index $\mathrm{o}-$ optimum) among all relative estimates such that is found

$$
\lambda^{o}=\max _{X \in S} \min _{k \in K} \lambda_{k}(X) .
$$

Using interrelation of expressions (9) and (10), we will transform a maximine problem (11) to an extreme problem 


$$
\begin{gathered}
\lambda^{o}=\max _{X \in S} \lambda, \\
\text { at restriction } \lambda \leq \lambda_{k}(X), k=\overline{1, K} .
\end{gathered}
$$

The resulting problem (12)-(13) let's call the $\lambda$-problem.

$\lambda$-problem (12)-(13) has $(N+1)$ dimension, as a consequence of the result of the solution of $\lambda$-problem (8)-(9) represents an optimum vector of $\boldsymbol{X}^{o} \in R^{N+1}$, $(N+1)$ which component an essence of the value of the $\lambda^{0}$, i.e. $\boldsymbol{X}^{o}=\left\{x_{1}^{o}, x_{2}^{o}, \cdots, x_{N}^{o}, x_{N+1}^{o}\right\}$, thus $x_{N+1}^{o}=\lambda^{o}$, and $(N+1)$ a component of a vector of $\boldsymbol{X}^{\boldsymbol{o}}$ selected in view of its specificity.

The received a pair of $\left\{\lambda^{o}, X^{o}\right\}=X^{o}$ characterizes the optimum solution of $\lambda$-problem (12)-(13) and according to vector problem of mathematical programming (5)-(8) with the equivalent criteria, solved on the basis of normalization of criteria and the principle of the guaranteed result. We will call in the optimum solution of $\boldsymbol{X}^{o}=\left\{\lambda^{o}, X^{\circ}\right\}, X^{o}$-an optimal point, and $\lambda^{\circ}-\mathrm{a}$ maximum level.

An important result of the algorithm for solving vector problems (5)-(8) with equivalent criteria is the following theorem.

Theorem 1. (The theorem of the two most contradictory criteria in the vector problem of mathematical programming with equivalent criteria).

In convex vector problems of mathematical programming at the equivalent criteria which is solved on the basis of normalization of criteria and the principle of the guaranteed result, in an optimum point of $\boldsymbol{X}^{o}=\left\{\lambda^{o}, X^{o}\right\}$ two criteria are always-denote their indexes $q \in \boldsymbol{K}, p \in \boldsymbol{K}$ (which in a sense are the most contradiction of the criteria $k=\overline{1, K}$ ), for which equality is carried out:

$$
\lambda^{o}=\lambda_{q}\left(X^{o}\right)=\lambda_{p}\left(X^{o}\right), q, p \in \boldsymbol{K}, X \in \boldsymbol{S},
$$

and other criteria are defined by inequalities:

$$
\lambda^{o} \leq \lambda_{k}\left(X^{o}\right), \forall k \in K, q \neq p \neq k .
$$

\subsection{Axioms and the Principle of Optimality of Vector Optimization with a Criterion Priority}

For development of methods of the solution of problems of vector optimization with a priority of criterion we use definitions as follows: priority of one criterion of vector problems, with a criterion priority over other criteria; numerical expression of a priority; the set priority of a criterion; the lower (minimum) level from all criteria with a priority of one of them; a subset of points with priority by criterion (Axiom 2); the principle of optimality of the solution of problems of vector optimization with the set priority of one of the criteria, and related theorems. For more details see [7] [13].

Definition 4. (About the priority of one criterion over the other).

The criterion of $q \in \boldsymbol{K}$ in the vector problem of Equations (12) and (13) in a point of $X \in S$ has priority over other criteria of $k=\overline{1, K}$, and the relative estimate of $\lambda_{q}(X)$ by this criterion is greater than or equal to relative estimates of $\lambda_{k}(X)$ of other criteria, i.e.: 


$$
\lambda_{q}(X) \geq \lambda_{k}(X), k=\overline{1, K}
$$

and a strict priority for at least one criterion of $t \in \boldsymbol{K}, \lambda_{q}(X)>\lambda_{k}(X), t \neq q$, and for other criteria of $\lambda_{q}(X) \geq \lambda_{k}(X), k=\overline{1, K}, k \neq t \neq q$.

Introduction of the definition of a priority of criterion $q \in \boldsymbol{K}$ in the vector problem of Equations (5)-(8) executed the redefinition of the early concept of a priority. Earlier the intuitive concept of the importance of this criterion was outlined, now this "importance" is defined as a mathematical concept: the higher the relative estimate of the $q$ th criterion compared to others, the more it is important (i.e., more priority), and the highest priority at a point of an optimum is $X_{k}^{*}, \forall q \in \boldsymbol{K}$.

From the definition of a priority of criterion of $q \in \boldsymbol{K}$ in the vector problem of Equations (5)-(8), it follows that it is possible to reveal a set of points $\boldsymbol{S}_{q} \subset \boldsymbol{S}$ that is characterized by $\lambda_{q}(X) \geq \lambda_{k}(X), k \neq q, X \in S_{q}$. However, the answer to whether a criterion of $q \in \boldsymbol{K}$ at a point of the set $S_{q}$ has more priority than others remains open. For clarification of this question, we define a communication coefficient between a couple of relative estimates of $q$ and $k$ that, in total, represent a vector:

$$
P^{q}(X)=\left\{p_{k}^{q}(X) \mid k=\overline{1, K}\right\}, q \in \boldsymbol{K}, \forall X \in \boldsymbol{S}_{q} .
$$

Definition 5. (About numerical expression of a priority of one criterion over another).

In the vector problem of Equations (12) and (13), with priority of the $q$ th criterion over other criteria of $k=\overline{1, K}$, for $\forall X \in S_{q}$, and a vector of $P^{q}(X)$ which shows how many times a relative estimate of $\lambda_{q}(X), q \in \boldsymbol{K}$, is more than other relative estimates of $\lambda_{k}(X), k=\overline{1, K}$, we define a numerical expression of the priority of the $q$ th criterion over other criteria of $k=\overline{1, K}$ as:

$$
\begin{aligned}
& P^{q}(X)=\left\{p_{k}^{q}(X)=\frac{\lambda_{q}(X)}{\lambda_{k}(X)}, k=\overline{1, K}\right\}, \\
& p_{k}^{q}(X) \geq 1, \forall X \in S_{q} \subset S, k=\overline{1, K}, \forall q \in \boldsymbol{K}
\end{aligned}
$$

Definition 6. (About the set numerical expression of a priority of one criterion over another).

In the vector problem of Equations (5)-(8) with a priority of criterion of $q \in \boldsymbol{K}$ for $\forall X \in S$, vector $P^{q}=\left\{p_{k}^{q}, k=\overline{1, K}\right\}$ is considered to be set by the person making decisions (i.e., decision-maker) if everyone is set a component of this vector. Set by the decision-maker, component $p_{k}^{q}$, from the point of view of the decision-maker, shows how many times a relative estimate of $\lambda_{q}(X), q \in \boldsymbol{K}$ is greater than other relative estimates of $\lambda_{k}(X), k=\overline{1, K}$. The vector of $p_{k}^{q}, k=\overline{1, K}$, is the numerical expression of the priority of the $q$ th criterion over other criteria of $k=\overline{1, K}$ :

$$
P^{q}(X)=\left\{p_{k}^{q}(X), k=\overline{1, K}\right\}, p_{k}^{q}(X) \geq 1, \forall X \in \boldsymbol{S}_{q} \subset S, k=\overline{1, K}, \forall q \in \boldsymbol{K}
$$

The vector problem of Equations (5)-(8), in which the priority of any criteria 
is set, is called a vector problem with the set priority of criterion. The problem of a task of a vector of priorities arises when it is necessary to determine the point $X^{o} \in S$ by the set vector of priorities. In the comparison of relative estimates with a priority of criterion of $q \in \boldsymbol{K}$, as well as in a task with equivalent criteria, we define the additional numerical characteristic of $\lambda$ which we call the level.

Definition 7. (About the lower level among all relative estimates with a criterion priority).

The $\lambda$ level is the lowest among all relative estimates with a priority of criterion of $q \in \boldsymbol{K}$ such that:

$$
\lambda \leq p_{k}^{q} \lambda_{k}(X), k=\overline{1, K}, q \in \boldsymbol{K}, \forall X \in \boldsymbol{S}_{q} \subset S ;
$$

The lower level for the performance of the condition in Equation (19) is defined as:

$$
\lambda=\min _{k \in K} p_{k}^{q} \lambda_{k}(X), q \in K, \forall X \in S_{q} \subset S .
$$

Equations (19) and (20) are interconnected and serve as a further transition from the operation of the definition of the minimum to restrictions, and vice versa. In Section 3.1, we gave the definition of a Pareto optimal point $X^{0} \in S$ with equivalent criteria. Considering this definition as an initial one, we will construct a number of the axioms dividing an admissible set of $\boldsymbol{S}$ into, first, a subset of Pareto optimal points $S^{\circ}$, and, secondly, a subset of points $S_{q} \subset S, q \in K$, with priority for the $q$ th criterion.

Axiom 2. (About a subset of points, priority by criterion).

In the vector problem of Equations (12)-(13), the subset of points $\boldsymbol{S}_{q} \subset \boldsymbol{S}$ is called the area of priority of criterion of $q \in \boldsymbol{K}$ over other criteria, if

$$
\forall X \in S_{q} \quad \forall k \in K \quad \lambda_{q}(X) \geq \lambda_{k}(X), \quad q \neq k .
$$

This definition extends to a set of Pareto optimal points $S^{o}$ that is given by the following definition.

Axiom 2a. (About a subset of points, priority by criterion, on Pareto's great number in a vector problem). In a vector problem of mathematical programming the subset of points $S_{q}^{o} \subset S^{o} \subset S$ is called the area of a priority of criterion of $q \in \boldsymbol{K}$ over other criteria, if $\forall X \in S_{q}^{o} \quad \forall k \in K \quad \lambda_{q}(X) \geq \lambda_{k}(X)$, $q \neq k$.

In the following we provide explanations.

Axiom 2 and 2a allow the breaking of the vector problem in Equations (5)-(8) into an admissible set of points $\mathcal{S}$, including a subset of Pareto optimal points, $S^{o} \subset S$, and subsets:

One subset of points $\boldsymbol{S}^{\prime} \subset \boldsymbol{S}$ where criteria are equivalent, and a subset of points of $\boldsymbol{S}^{\prime}$ crossed with a subset of points $\boldsymbol{S}^{o}$, allocated to a subset of Pareto optimal points at equivalent criteria $\boldsymbol{S}^{o o}=\boldsymbol{S}^{\prime} \cap \boldsymbol{S}^{o}$. As will be shown further, this consists of one point of $X^{\circ} \in S$, i.e.

$$
X^{o}=S^{o o}=S^{\prime} \cap S^{o}, S^{\prime} \in S, S^{o} \subset S .
$$

" $K$ " subsets of points where each criterion of $q=\overline{1, K}$ has a priority over 
other criteria of $k=\overline{1, K}, q \neq k$, and thus breaks, first, sets of all admissible points $S$, into subsets $S_{q} \subset S, q=\overline{1, K}$ and, second, a set of Pareto optimal points, $S^{o}$, into subsets $S_{q}^{o} \subset \boldsymbol{S}^{o} \subset \boldsymbol{S}, q=\overline{1, K}$. This yields:

$$
\boldsymbol{S}^{\prime} \cup\left(\bigcup_{q \in K} \boldsymbol{S}_{q}^{o}\right) \equiv \boldsymbol{S}^{o}, \boldsymbol{S}_{q}^{o} \subset \boldsymbol{S}^{o} \subset \boldsymbol{S}, q=\overline{1, K} .
$$

We note that the subset of points $S_{q}^{o}$, on the one hand, is included in the area (a subset of points) of priority of criterion of $q \in \boldsymbol{K}$ over other criteria: $S_{q}^{o} \subset S_{q} \subset S$, and, on the other, in a subset of Pareto optimal points $S_{q}^{o} \subset S^{o} \subset S$.

Axiom 2 and the numerical expression of priority of criterion (Definition 5) allow the identification of each admissible point of $X \in S$ (by means of vector $P^{q}(X)=\left\{p_{k}^{q}(X)=\frac{\lambda_{q}(X)}{\lambda_{k}(X)}, k=\overline{1, K}\right\}$, to form and choose:

a subset of points by priority criterion $S_{q}$, which is included in a set of points $\mathcal{S}, \forall q \in \boldsymbol{K} \quad X \in \boldsymbol{S}_{q} \subset \boldsymbol{S}$, (such a subset of points can be used in problems of clustering, but is beyond this article);

a subset of points by priority criterion $S_{q}^{o}$, which is included in a set of Pareto optimal points $\boldsymbol{S}^{o}, \forall q \in K, X \in \boldsymbol{S}_{q}^{o} \subset \boldsymbol{S}^{o}$.

Thus, full identification of all points in the vector problem of Equations (12) and (13) is executed in sequence as:

$$
\begin{aligned}
& \begin{array}{lccc}
\text { Set of admissible } & \begin{array}{c}
\text { Subset of points, } \\
\text { optimum across }
\end{array} & \begin{array}{c}
\text { Subset of points, } \\
\text { optimum across }
\end{array} & \text { Separate point of a } \\
\text { points of } X \in S & \text { Pareto } & \forall X \in S
\end{array} \\
& \begin{array}{cccc}
\text { points of } X \in S & \text { Pareto, } & \text { Pareto } & \forall X \in S \\
\rightarrow & X \in S^{o} \subset S \rightarrow & X \in \boldsymbol{S}_{q}^{o} \subset \boldsymbol{S}^{o} \subset \boldsymbol{S} & X \in \boldsymbol{S}_{q}^{o} \subset \boldsymbol{S}^{o} \subset \boldsymbol{S}
\end{array}
\end{aligned}
$$

This is the most important result which allows the output of the principle of optimality and to construct methods of a choice of any point of Pareto's great number.

Definition 8. (Principle of optimality 2. The solution of a vector problem with the set criterion priority).

The vector problem of Equations (12) and (13) with the set priority of the qth criterion of $p_{k}^{q} \lambda_{k}(X), k=\overline{1, K}$ is considered solved if the point $X^{o}$ and maximum level $\lambda^{o}$ among all relative estimates is found such that:

$$
\lambda^{o}=\max _{X \in S} \min _{k \in K} p_{k}^{q} \lambda_{k}(X), q \in K .
$$

Using the interrelation of Equations (19) and (20), we can transform the maximine problem of Equation (33) into an extreme problem of the form:

$$
\lambda^{o}=\max _{X \in S} \lambda,
$$

at restriction $\lambda \leq p_{k}^{q} \lambda_{k}(X), k=\overline{1, K}$.

We call Equations (22) and (23) the $\lambda$-problem with a priority of the $q$ th criterion. 
The solution of the $\lambda$-problem is the point $\boldsymbol{X}^{o}=\left\{X^{o}, \lambda^{o}\right\}$. This is also the result of the solution of the vector problem of Equations (5)-(8) with the set priority of the criterion, solved on the basis of normalization of criteria and the principle of the guaranteed result.

In the optimum solution $X^{o}=\left\{X^{o}, \lambda^{\circ}\right\}, X^{o}$, an optimum point, and $\lambda^{o}$, the maximum bottom level, the point of $X^{o}$ and the $\lambda^{o}$ level correspond to restrictions of Equation (15), which can be written as:

$$
\lambda^{o} \leq p_{k}^{q} \lambda_{k}\left(X^{o}\right), k=\overline{1, K} .
$$

These restrictions are the basis of an assessment of the correctness of the results of a decision in practical vector problems of optimization.

From Definitions 1 and 2, "Principles of optimality", follows the opportunity to formulate the concept of the operation "opt".

Definition 9. (Mathematical operation “opt”).

In the vector problem of Equations (1)-(4), in which "max" and "min" are part of the criteria, the mathematical operation "opt" consists of the definition of a point $X^{\circ}$ and the maximum $\lambda^{\circ}$ bottom level to which all criteria measured in relative units are lifted:

$$
\lambda^{o} \leq \lambda_{k}\left(X^{o}\right)=\frac{f_{k}(X)-f_{k}^{0}}{f_{k}^{*}-f_{k}^{0}}, k=\overline{1, K},
$$

i.e., all criteria of $\lambda_{k}\left(X^{o}\right), k=\overline{1, K}$, are equal to or greater than the maximum level of $\lambda^{o}$ (therefore $\lambda^{o}$ is also called the guaranteed result).

Theorem 2. (The theorem of the most inconsistent criteria in a vector problem with the set priority).

If in the convex vector problem of mathematical programming of Equations (5)-(8) the priority of the $q$ th criterion of $p_{k}^{q}, k=\overline{1, K}, \forall q \in K$ over other criteria is set, at a point of an optimum $X^{o} \in \boldsymbol{S}$ obtained on the basis of normalization of criteria and the principle of guaranteed result, there will always be two criteria with the indexes $r \in \boldsymbol{K}, t \in \boldsymbol{K}$, for which the following strict equality holds:

$$
\lambda^{o}=p_{k}^{r} \lambda_{r}\left(X^{o}\right)=p_{k}^{t} \lambda_{t}\left(X^{o}\right), r, t \in K,
$$

and other criteria are defined by inequalities:

$$
\lambda^{o} \leq p_{k}^{q}\left(X^{o}\right), k=\overline{1, K}, \forall q \in K, q \neq r \neq t
$$

Criteria with the indexes $r \in \boldsymbol{K}, t \in \boldsymbol{K}$ for which the equality of Equation (38) holds are called the most inconsistent.

Proof. Similar to Theorem 2 [7].

We note that in Equations (25) and (26), the indexes of criteria $r, t \in \boldsymbol{K}$ can coincide with the $q \in \boldsymbol{K}$ index.

Consequence of Theorem 1, about equality of an optimum level and relative estimates in a vector problem with two criteria with a priority of one of them.

In a convex vector problem of mathematical programming with two equiva- 
lent criteria, solved on the basis of normalization of criteria and the principle of the guaranteed result, at an optimum point $X^{0}$ equality is always carried out at a priority of the first criterion over the second:

$$
\lambda^{o}=\lambda_{1}\left(X^{o}\right)=p_{2}^{1}\left(X^{o}\right) \lambda_{2}\left(X^{o}\right), X^{o} \in S,
$$

where

$$
p_{2}^{1}\left(X^{o}\right)=\lambda_{1}\left(X^{o}\right) / \lambda_{2}\left(X^{o}\right),
$$

and at a priority of the second criterion over the first:

$$
\lambda^{o}=\lambda_{2}\left(X^{o}\right)=p_{1}^{2}\left(X^{o}\right) \lambda_{1}\left(X^{o}\right), X^{o} \in S, \text { where } p_{1}^{2}\left(X^{o}\right)=\lambda_{2}\left(X^{o}\right) / \lambda_{1}\left(X^{o}\right) .
$$

\subsection{Mathematical Algorithm of the Solution of a Vector Problem with Equivalent Criteria}

To solve of the vector problems of mathematical programming (5)-(8) the methods based on axiomatic of the normalization of criteria and the principle of the guaranteed result [1] [16] are offered. Methods follow from an axiom 1 and the principle of optimality 1 . We will present in the form of a number of steps:

Step 1. The problem (5)-(8) by each criterion separately is solved, i.e. for $\forall k \in \boldsymbol{K}_{1}$ is solved at the maximum, and for $\forall k \in \boldsymbol{K}_{2}$ is solved at a minimum. As a result of the decision we will receive: $X_{k}^{*}$-an optimum point by the corresponding criterion, $k=\overline{1, K} ; f_{k}^{*}=f_{k}\left(X_{k}^{*}\right)$-the criterion size $k$ th in this point, $k=\overline{1, K}$.

Step 2. We define the worst value of each criterion on $S: f_{k}^{0}, k=\overline{1, K}$. For what the problem (5)-(8) for each criterion of $k=\overline{1, K_{1}}$ on a minimum is solved:

$$
f_{k}^{0}=\min f_{k}(X), G(X) \leq B, X \geq 0, k=\overline{1, K_{1}} .
$$

The problem (5)-(8) for each criterion $k=\overline{1, K_{2}}$ maximum is solved:

$$
f_{k}^{0}=\max f_{k}(X), G(X) \leq B, X \geq 0, k=\overline{1, K_{2}} .
$$

As a result of the decision we will receive: $X_{k}^{0}=\left\{x_{j}, j=\overline{1, N}\right\}$-an optimum point by the corresponding criterion, $k=\overline{1, K} ; f_{k}^{0}=f_{k}\left(X_{k}^{0}\right)$-the criterion size $k$ th a point, $X_{k}^{0}, k=\overline{1, K}$.

Step 3. The system analysis of a set of points, optimum across Pareto, for this purpose in optimum points of $X^{*}=\left\{X_{k}^{*}, k=\overline{1, K}\right\}$, are defined sizes of criterion functions of $F\left(X^{*}\right)$ and relative estimates $\lambda\left(X^{*}\right)$,

$$
\begin{aligned}
\lambda_{k}(X)=\frac{f_{k}(X)-f_{k}^{0}}{f_{k}^{*}-f_{k}^{0}}, \forall k \in K: \\
F\left(X^{*}\right)=\left\{f_{k}\left(X_{k}^{*}\right), q=\overline{1, K}, k=\overline{1, K}\right\}=\left|\begin{array}{c}
f_{1}\left(X_{1}^{*}\right), \cdots, f_{K}\left(X_{1}^{*}\right) \\
\vdots \\
f_{1}\left(X_{K}^{*}\right), \cdots, f_{K}\left(X_{K}^{*}\right)
\end{array}\right|,
\end{aligned}
$$




$$
\lambda\left(X^{*}\right)=\left\{\lambda_{q}\left(X_{k}^{*}\right), q=\overline{1, K}, k=\overline{1, K}\right\}=\left|\begin{array}{c}
\lambda_{1}\left(X_{1}^{*}\right), \cdots, \lambda_{K}\left(X_{1}^{*}\right) \\
\vdots \\
\lambda_{1}\left(X_{K}^{*}\right), \cdots, \lambda_{K}\left(X_{K}^{*}\right)
\end{array}\right| .
$$

As a whole on a problem $\forall k \in \boldsymbol{K}$ the relative assessment of $\lambda_{k}(X), k=\overline{1, K}$ lies within

$$
0 \leq \lambda_{k}(X) \leq 1, k=\overline{1, K} .
$$

Step 4. Creation of the $\lambda$-problem.

Creation of $\lambda$-problem is carried out in two stages:

initially built the maximine problem of optimization with the normalized criteria which at the second stage will be transformed to the standard problem of mathematical programming called $\lambda$-problem.

For construction maximine a problem of optimization we use definition 2-relative level:

$$
\forall X \in S \quad \lambda=\min _{k \in K} \lambda_{k}(X) .
$$

The bottom $\lambda$ level is maximized on $X \in S$, as a result we will receive a maximine problem of optimization with the normalized criteria.

$$
\lambda^{o}=\max _{X \in S} \min _{k \in K} \lambda_{k}(X) .
$$

At the second stage we will transform a problem (29) to a standard problem of mathematical programming:

$$
\begin{gathered}
\lambda^{o}=\max _{X \in S} \lambda, \lambda^{o}=\max _{X \in S} \lambda, \\
\lambda-\lambda_{k}(X) \leq 0, k=\overline{1, K}, \rightarrow \lambda-\frac{f_{k}(X)-f_{k}^{0}}{f_{k}^{*}-f_{k}^{0}} \leq 0, k=\overline{1, K}, \\
G(X) \leq B, X \geq 0, G(X) \geq B, X \geq 0,
\end{gathered}
$$

where the vector of unknown of $X$ has dimension of $N+1: \quad X=\left\{\lambda, x_{1}, \cdots, x_{N}\right\}$.

Step 5. Solution of $\lambda$-problem.

$\lambda$-problem (30)-(32) is a standard problem of convex programming and for its decision standard methods are used.

As a result of the solution of $\lambda$-problem it is received: $X^{o}=\left\{X^{\circ}, \lambda^{\circ}\right\}-$ an optimum point;

$$
\begin{aligned}
& f_{k}\left(X^{o}\right), k=\overline{1, K}-\text { values of the criteria in this point; } \\
& \lambda_{k}\left(X^{o}\right)=\frac{f_{k}(X)-f_{k}^{0}}{f_{k}^{*}-f_{k}^{0}}, k=\overline{1, K} \text {-sizes of relative estimates; }
\end{aligned}
$$

$\lambda^{\circ}$-the maximum relative estimates which is the maximum bottom level for all relative estimates of $\lambda_{k}\left(X^{o}\right)$, or the guaranteed result in relative units. $\lambda^{o}$ guarantees that all relative estimates of $\lambda_{k}\left(X^{o}\right)$ more or are equal $\lambda^{o}$ :

$$
\lambda_{k}\left(X^{o}\right)^{o}, k=\overline{1, K} \text { or } \lambda^{o} \leq \lambda_{k}\left(X^{o}\right), k=\overline{1, K}, X^{o} \in S,
$$

and according to Theorem 1 [1] [16] point of $X^{o}=\left\{\lambda^{o}, x_{1}, \cdots, x_{N}\right\}$ is optimum across Pareto. 


\subsection{Mathematical Method of the Solution of a Vector Problem with Criterion Priority}

(Method of the decision in problems of vector optimization with a criterion priority) [7].

Step 1. We solve a vector problem with equivalent criteria. The algorithm of the decision is presented in Section 4.3.

As a result of the decision we obtain:

Optimum points by each criterion separately $X_{k}^{*}, k=\overline{1, K}$ and sizes of criterion functions in these points of $f_{k}^{*}=f_{k}\left(X_{k}^{*}\right), k=\overline{1, K}$, which represent the boundary of a set of Pareto optimal points;

Anti-optimum points by each criterion of $X_{k}^{0}=\left\{x_{j}, j=\overline{1, N}\right\}$ and the worst unchangeable part of each criterion of $f_{k}^{0}=f_{k}\left(X_{k}^{0}\right), k=\overline{1, K}$;

$\boldsymbol{X}^{o}=\left\{X^{0}, \lambda^{\circ}\right\}$, an optimum point, as a result of the solution of VPMP at equivalent criteria, i.e., the result of the solution of a maximine problem and the $\lambda$-problem constructed on its basis;

$\lambda^{o}$, the maximum relative assessment which is the maximum lower level for all relative estimates of $\lambda_{k}\left(X^{\circ}\right)$, or the guaranteed result in relative units, $\lambda^{\circ}$ guarantees that all relative estimates of $\lambda_{k}\left(X^{0}\right)$ are equal to or greater than $\lambda^{o}$ :

$$
\lambda^{o} \leq \lambda_{k}\left(X^{o}\right), k=\overline{1, K}, X^{o} \in S .
$$

The person making the decision carries out the analysis of the results of the solution of the vector problem with equivalent criteria. If the received results satisfy the decision maker, then the process concludes, otherwise subsequent calculations are performed.

In addition, we calculate:

in each point $X_{k}^{*}, k=\overline{1, K}$ we determine sizes of all criteria of: $q=\overline{1, K}$ $\left\{f_{q}\left(X_{k}^{*}\right), q=\overline{1, K}\right\}, k=\overline{1, K}$, and relative estimates

$$
\begin{gathered}
\lambda\left(X^{*}\right)=\left\{\lambda_{q}\left(X_{k}^{*}\right), q=\overline{1, K}, k=\overline{1, K}\right\}, \quad \lambda_{k}(X)=\frac{f_{k}(X)-f_{k}^{0}}{f_{k}^{*}-f_{k}^{0}}, \forall k \in K: \\
F\left(X^{*}\right)=\left|\begin{array}{c}
f_{1}\left(X_{1}^{*}\right), \cdots, f_{K}\left(X_{1}^{*}\right) \\
\vdots \\
f_{1}\left(X_{K}^{*}\right), \cdots, f_{K}\left(X_{K}^{*}\right)
\end{array}\right|, \lambda\left(X^{*}\right)=\left|\begin{array}{c}
\lambda_{1}\left(X_{1}^{*}\right), \cdots, \lambda_{K}\left(X_{1}^{*}\right) \\
\vdots \\
\lambda_{1}\left(X_{K}^{*}\right), \cdots, \lambda_{K}\left(X_{K}^{*}\right)
\end{array}\right| .
\end{gathered}
$$

Matrices of criteria of $F\left(X^{*}\right)$ and relative estimates of $\lambda\left(X^{*}\right)$ show the sizes of each criterion of $k=\overline{1, K}$ upon transition from one optimum point $X_{k}^{*}, k \in K$ to another $X_{q}^{*}, q \in \boldsymbol{K}$, i.e., on the border of a great number of Pareto.

atan optimum point at equivalent criteria $X^{o}$ we calculate sizes of criteria and relative estimates:

$$
f_{k}\left(X^{o}\right), k=\overline{1, K} ; \quad \lambda_{k}\left(X^{o}\right), k=\overline{1, K},
$$

which satisfy the inequality of Equation (33). In other points $X \in \boldsymbol{S}^{o}$, in relative units the criteria of $\lambda=\min _{k \in K} \lambda_{k}(X)$ are always less than $\lambda^{o}$, given the $\lambda$-problem of Equations (30)-(32). 
This information is also a basis for further study of the structure of a great number of Pareto.

Step 2. Choice of priority criterion of $q \in \boldsymbol{K}$.

From theory (see Theorem 1) it is known that at an optimum point $X^{o}$ there are always two most inconsistent criteria, $q \in \boldsymbol{K}$ and $v \in \boldsymbol{K}$, for which in relative units an exact equality holds:

$$
\begin{gathered}
\lambda^{o}=\lambda_{q}\left(X^{o}\right)=\lambda_{v}\left(X^{o}\right), q, v \in \boldsymbol{K}, X \in \boldsymbol{S} \text {. Others are subject to inequalities: } \\
\lambda^{o} \leq \lambda_{k}\left(X^{o}\right), \forall k \in \boldsymbol{K}, q \neq v \neq k
\end{gathered}
$$

As a rule, the criterion which the decision-maker would like to improve is part of this couple, and such a criterion is called a priority criterion, which we designate $q \in \boldsymbol{K}$.

Step 3. Numerical limits of the change of the size of a priority of criterion $q \in \boldsymbol{K}$ are defined.

For priority criterion $q \in \boldsymbol{K}$ from the matrix of Equation (34) we define the numerical limits of the change of the size of criterion:

in physical units of $f_{k}\left(X^{o}\right) \leq f_{q}(X) \leq f_{q}\left(X_{q}^{*}\right), k \in \boldsymbol{K}$,

where $f_{q}\left(X_{q}^{*}\right)$ derives from the matrix of Equation (34) $F\left(X^{*}\right)$, all criteria showing sizes measured in physical units, $f_{q}\left(X^{o}\right)$ from Equation (35), and,

$$
\text { in relative units of } \lambda_{k}\left(X^{o}\right) \leq \lambda_{q}(X) \leq \lambda_{q}\left(X_{q}^{*}\right), k \in \boldsymbol{K},
$$

where $\lambda_{q}\left(X_{q}^{*}\right)$ derives from the matrix $\lambda\left(X^{*}\right)$, all criteria showing sizes measured in relative units (we note that $\lambda_{q}\left(X_{q}^{*}\right)=1$ ), $\lambda_{q}\left(X^{o}\right)$ from Equation (34).

As a rule, Equations (36) and (37) are given for the display of the analysis.

Step 4. Choice of the size of priority criterion (decision-making).

The person making the decision carries out the analysis of the results of calculations of Equation (34) and from the inequality of Equation (36) chooses the numerical size $f_{q}$ of the criterion of $q \in \boldsymbol{K}$ :

$$
f_{q}\left(X^{o}\right) \leq f_{q} \leq f_{q}\left(X_{q}^{*}\right), q \in \boldsymbol{K} .
$$

For the chosen size of the criterion of $f_{q}$ it is necessary to define a vector of unknown $X^{\circ}$. For this purpose, we carry out the subsequent calculations.

Step 5. Calculation of a relative assessment.

For the chosen size of the priority criterion of $f_{q}$ the relative assessment is calculated as:

$$
\lambda_{q}=\frac{f_{q}-f_{q}^{0}}{f_{q}^{*}-f_{q}^{0}},
$$

which upon transition from point $X^{o}$ to $X_{q}^{*}$, according to Equation (32), lies in the limits:

$$
\lambda_{q}\left(X^{o}\right) \leq \lambda_{q} \leq \lambda_{q}\left(X_{q}^{*}\right)=1
$$

Step 6. Calculation of the coefficient of linear approximation. 
Assuming a linear nature of the change of criterion of $f_{q}(X)$ in Equation (36) and according to the relative assessment of $\lambda_{q}(X)$ in Equation (37), using standard methods of linear approximation we calculate the proportionality coefficient between $\lambda_{q}\left(X^{o}\right), \lambda_{q}$, which we call $\rho$ :

$$
\rho=\frac{\lambda_{q}-\lambda_{q}\left(X^{o}\right)}{\lambda_{q}^{*}-\lambda_{q}^{0}}, q \in \boldsymbol{K} .
$$

Step 7. Calculation of coordinates of priority criterion with the size $f_{q}$. In accordance with Equation (38), the coordinates of the $X^{q}$ priority criterion point lie within the following limits: $\rho=\frac{\lambda_{q}-\lambda_{q}\left(X^{o}\right)}{\lambda_{q}^{*}-\lambda_{q}^{0}}, q \in \boldsymbol{K}$. Assuming a linear nature of change of the vector $X_{q}=\left\{x_{1}^{q}, \cdots, x_{N}^{q}\right\}$ we determine coordinates of a point of priority criterion with the size $f_{q}$ with the relative assessment of Equation (39):

$$
X_{q}=\left\{x_{1}^{q}=x_{1}^{o}+\left(x_{q}^{*}(1)-x_{1}^{o}\right), \cdots, x_{N}^{q}=x_{N}^{o}+\left(x_{q}^{*}(N)-x_{N}^{o}\right)\right\},
$$

where $X^{o}=\left\{x_{1}^{o}, \cdots, x_{N}^{o}\right\}, X_{q}^{*}=\left\{x_{q}^{*}(1), \cdots, x_{q}^{*}(N)\right\}$.

Step 8. Calculation of the main indicators of a point $x_{q}$.

For the obtained point $x_{q}$, we calculate:

all criteria in physical units $F^{q}=\left\{f_{k}\left(x^{q}\right), k=\overline{1, K}\right\}$;

all relative estimates of criteria $\lambda^{q}=\left\{\lambda_{k}^{q}, k=\overline{1, K}\right\}$,

$\lambda_{k}\left(x^{q}\right)=\frac{f_{k}\left(x^{q}\right)-f_{k}^{0}}{f_{k}^{*}-f_{k}^{0}}, k=\overline{1, K}$;

the vector of priorities $P^{q}=\left\{p_{k}^{q}=\frac{\lambda_{q}\left(x^{q}\right)}{\lambda_{k}\left(x^{q}\right)}, k=\overline{1, K}\right\}$;

the maximum relative assessment $\lambda^{o q}=\min \left(p_{k}^{q} \lambda_{k}\left(x^{q}\right), k=\overline{1, K}\right)$.

Any point from Pareto's set $\boldsymbol{X}_{t}^{o}=\left\{\lambda_{t}^{o}, X_{t}^{o}\right\} \in \boldsymbol{S}^{o}$ can be similarly calculated.

Analysis of results. The calculated size of criterion $f_{q}\left(X_{t}^{o}\right), q \in \boldsymbol{K}$ is usually not equal to the set $f_{q}$. The error of the choice of $f_{q}=\left|f_{q}\left(X_{t}^{o}\right)-f_{q}\right|$ is defined by the error of linear approximation.

\section{Methodology for Selecting Optimal Parameters of Engineering Systems under Conditions of Certainty and Uncertainty Based on Vector Optimization}

As the object of the study we consider "Engineering Systems," for which the construction of a mathematical model for three types of engineering problems in the form of a vector problem in the second section is presented. The engineering system study is carried out, first, under conditions of certainty, when data on functional characteristics of the engineering system are known; second, in uncertainty conditions where discrete values of individual characteristics are known; there are also known data on limitations imposed on the operation of the system. Mathematical apparatus of engineering system modeling is based on theory and methods 
of vector optimization, which are presented in the third section. In organizational terms, the process of modeling and simulation of the technical system is presented in the form of a methodology: "Methodology of selection of optimal parameters of engineering systems in conditions of certainty and uncertainty."

The methodology includes a number of stages.

1) Formation of the technical specification (source data) for numerical modeling and choice of optimum parameters of a system. The initial data is formed by the designer who projects the system.

2) Construction of mathematical and numerical models of the technical system in terms of certainty and uncertainty.

3) The solution of the vector problem of mathematical programming (VPMP) - a model of the Engineering system at equivalent criteria.

4) Creation of geometrical interpretation of results of the decision in a three-dimensional coordinate system in relative units.

5) The solution of a vector problem of mathematical programming-a model of theEngineering system at the given priority of the criterion.

6) Geometrical interpretation of results of the decision in a three-dimensional coordinate system in physical units.

\subsection{Methodology for Selecting of the Optimal Parameters of Technical System under Conditions of Certainty and Uncertainty Based on Vector Optimization}

The problem of numerical modeling and simulation of a technical system in which data on a certain set of functional characteristics (conditions of certainty), discrete values of characteristics (conditions of uncertainty) and restrictions imposed on the functioning of the technical system are known is considered [2] [5] [8] [16]. The numerical problem of modeling a technical system is considered with equivalent criteria and with a given criterion priority.

\section{Stage 1. Formation of technical specifications (initial data)}

It is given. We're investigating the technical (engineering) system. The functioning of the technical system is determined by four parameters $X=\left\{x_{1}, x_{2}, x_{3}, x_{4}\right\}$, which represent the vector of controlled variables. The parameters of the technical system are set within the following limits:

$$
22 \leq x_{1} \leq 88,0 \leq x_{2} \leq 66,2.2 \leq x_{3} \leq 8.8,2.2 \leq x_{4} \leq 8.8 .
$$

The operation of the technical system is determined by four characteristics (criteria):

$$
F(X)=\left\{f_{1}(X), f_{2}(X), f_{3}(X), f_{4}(X)\right\}, \text { which size of an assessment depends }
$$
on a vector of $X$.

The conditions of certainty. For four characteristics of $f_{4}(X)$ and $f_{3}(X)$ functional dependence on parameters $X=\left\{x_{j}, j=\overline{1, N}, N=4\right\}$ is known.

$$
\begin{aligned}
f_{4}(X)= & 19.253-0.0081 * x_{1}-0.7005 * x_{2}-0.3605 * x_{3}+0.9769 * x_{4} \\
& +0.0126 * x_{1} * x_{2}+0.0644 * x_{1} * x_{3}-0 * x_{1} * x_{4}+0.0396 * x_{2} * x_{3} \\
& +0.0002 * x_{2} * x_{4}+0.0004 * x_{3} * x_{4}-0.0016 * x_{1}^{2}+0.0027 * x_{2}^{2} \\
& +0.0045 * x_{3}^{2}-0.0235 * x_{4}^{2}
\end{aligned}
$$


The uncertainty condition. For the first, second and third characteristic the results of experimental data are known: the values of the parameters and corresponding characteristics. Numerical values of parameters $X$ and characteristics of $y_{1}(X), y_{2}(X)$ and $y_{3}(X)$ are presented in Table 1 .

Decision, assessment size of the first and the third characteristic (criterion) is possible to receive above: $f_{1}(X) \rightarrow \max f_{3}(X) \rightarrow \max$; for the second and fourth characteristic is possible below:

$y_{2}(X) \rightarrow \min \quad f_{4}(X) \rightarrow \min$. Parameters $X=\left\{x_{1}, x_{2}, x_{3}, x_{4}\right\}$ change in the following limits:

$$
x_{1} \in[225588], x_{2} \in\left[\begin{array}{ll}
0 & 3366
\end{array}\right], x_{3} \in[2.25 .58 .8], x_{4} \in[2.25 .58 .8]
$$

It is required. To construct model of the system in the form of a vector problem. To solve a vector problem with equivalent criteria. To choose priority criterion. To establish numerical value of priority criterion. To make the best decision (optimum) with a specified priority criterion.

Note. The author developed in the Matlab system the software for the decision of vector problem of mathematical programming. The vector problem includes four variables (parameters of technical system): $X=\left\{x_{1}, x_{2}, x_{3}, x_{4}\right\}$ and four criteria (characteristic) of $F(X)=\left\{f_{1}(X), f_{2}(X), f_{3}(X), f_{4}(X)\right\}$. But for each new data (new system) the program is configured individually. In the software criteria of $F(X)=\left\{f_{1}(X), f_{2}(X), \cdots, f_{6}(X)\right\}$ with uncertainty conditions (in Table 1 they are provided as a part of $\left.\left\{y_{1}, y_{2}, y_{3}, y_{4}\right\}\right)$ can change from zero (i.e. all criteria are constructed in the conditions of determinacy) to six (i.e. all criteria are constructed in the conditions of uncertainty).

Stage 2. Creation of mathematical and numerical model of the system in the conditions of definiteness and indeterminacy

The Creating a numerical model of the system includes the following sections: Choosing a mathematical model of the system; Building a model in certainty conditions; Construction in the conditions of not certainty; Construction of a numerical model of the system under certainty and uncertainty.

Mathematical model of the system

We will present model of the system in the conditions of definiteness and uncertainty in total:

$$
\begin{gathered}
\text { Opt } F(X)=\left\{\max F_{1}(X)=\left\{\max f_{k}(X), k=\overline{1, K_{1}^{\text {def }}}\right\},\right. \\
\left.\max I_{1}(X) \equiv\left\{\max f_{k}\left(X_{i}, i=\overline{1, M}\right)\right\}^{\mathrm{T}}, k=\overline{1, K_{1}^{\text {unc }}}\right\}, \\
\min F_{2}(X)=\left\{\min f_{k}(X), k=\overline{1, K_{2}^{\text {def }}},\right. \\
\left.\left.\min I_{2}(X) \equiv\left\{\min f_{k}\left(X_{i}, i=\overline{1, M}\right)\right\}^{\mathrm{T}}, k=\overline{1, K_{2}^{\text {unc }}}\right\}\right\}, \\
\text { at restriction } f_{k}^{\min } \leq f_{k}(X) \leq f_{k}^{\max }, k=\overline{1, K}, x_{j}^{\min } \leq x_{j} \leq x_{j}^{\max }, j=\overline{1, N},
\end{gathered}
$$


Table 1. Numerical values of parameters and characteristics of the system.

\begin{tabular}{|c|c|c|c|c|c|c|}
\hline$x_{1}$ & $x_{2}$ & $x_{3}$ & $x_{4}$ & $y_{1}(X) \rightarrow \max$ & $y_{2}(X) \rightarrow \min$ & $y_{3}(X) \rightarrow \max$ \\
\hline 22 & 0 & 2.2 & 2.2 & 363.0 & 1053.8 & 47.7 \\
\hline 22 & 0 & 2.2 & 5.5 & 374.0 & 1067.0 & 47.3 \\
\hline 22 & 0 & 2.2 & 8.8 & 382.8 & 1078.0 & 47.2 \\
\hline 22 & 0 & 5.5 & 2.2 & 388.3 & 1111.0 & 50.7 \\
\hline 22 & 0 & 5.5 & 5.5 & 396.0 & 1155.0 & 46.8 \\
\hline 22 & 0 & 5.5 & 8.8 & 401.5 & 1152.8 & 46.3 \\
\hline 22 & 0 & 8.8 & 2.2 & 413.6 & 1151.7 & 44.2 \\
\hline 22 & 0 & 8.8 & 5.5 & 419.1 & 1148.4 & 43.0 \\
\hline 22 & 0 & 8.8 & 8.8 & 429.0 & 1147.3 & 42.5 \\
\hline 22 & 33 & 2.2 & 2.2 & 275.0 & 1964.6 & 58.3 \\
\hline 22 & 33 & 2.2 & 5.5 & 283.8 & 1974.5 & 57.5 \\
\hline 22 & 33 & 2.2 & 8.8 & 289.3 & 1983.3 & 57.1 \\
\hline 22 & 33 & 5.5 & 2.2 & 297.0 & 1995.4 & 56.5 \\
\hline 22 & 33 & 5.5 & 5.5 & 306.9 & 2003.1 & 55.1 \\
\hline 22 & 33 & 5.5 & 8.8 & 311.3 & 2015.2 & 54.9 \\
\hline 22 & 33 & 8.8 & 2.2 & 319.0 & 2027.3 & 54.8 \\
\hline 22 & 33 & 8.8 & 5.5 & 331.1 & 2046.0 & 52.8 \\
\hline 22 & 33 & 8.8 & 8.8 & 341.0 & 2058.1 & 53.0 \\
\hline 22 & 66 & 2.2 & 2.2 & 187.0 & 2708.2 & 75.9 \\
\hline 22 & 66 & 2.2 & 5.5 & 192.5 & 2585.0 & 71.5 \\
\hline 22 & 66 & 2.2 & 8.8 & 203.5 & 2541.0 & 68.2 \\
\hline 22 & 66 & 5.5 & 2.2 & 209.0 & 2519.0 & 66.4 \\
\hline 22 & 66 & 5.5 & 5.5 & 217.8 & 2596.0 & 68.2 \\
\hline 22 & 66 & 5.5 & 8.8 & 224.4 & 2662.0 & 70.4 \\
\hline 22 & 66 & 8.8 & 2.2 & 231.0 & 2770.9 & 72.4 \\
\hline 22 & 66 & 8.8 & 5.5 & 243.1 & 2783.0 & 71.5 \\
\hline 22 & 66 & 8.8 & 8.8 & 253.0 & 2801.7 & 70.6 \\
\hline 55 & 0 & 2.2 & 2.2 & 11.0 & 3284.6 & 100.5 \\
\hline 55 & 0 & 2.2 & 5.5 & 17.6 & 3301.1 & 100.1 \\
\hline 55 & 0 & 2.2 & 8.8 & 26.4 & 3307.7 & 99.0 \\
\hline 55 & 0 & 5.5 & 2.2 & 33.0 & 3315.4 & 98.8 \\
\hline 55 & 0 & 5.5 & 5.5 & 41.8 & 3320.9 & 97.9 \\
\hline 55 & 0 & 5.5 & 8.8 & 48.4 & 3334.1 & 97.6 \\
\hline 55 & 0 & 8.8 & 2.2 & 55.0 & 3347.3 & 97.0 \\
\hline 55 & 0 & 8.8 & 5.5 & 66.0 & 3366.0 & 95.7 \\
\hline 55 & 0 & 8.8 & 8.8 & 77.0 & 3378.1 & 95.3 \\
\hline 55 & 33 & 2.2 & 2.2 & 451.0 & 1095.6 & 54.6 \\
\hline 55 & 33 & 2.2 & 5.5 & 458.7 & 1111.0 & 50.6 \\
\hline 55 & 33 & 2.2 & 8.8 & 465.3 & 1133.0 & 48.4 \\
\hline 55 & 33 & 5.5 & 2.2 & 473.0 & 1147.3 & 47.7 \\
\hline 55 & 33 & 5.5 & 5.5 & 482.9 & 1166.0 & 46.2 \\
\hline 55 & 33 & 5.5 & 8.8 & 488.4 & 1188.0 & 45.1 \\
\hline
\end{tabular}




\section{Continued}

\begin{tabular}{|c|c|c|c|c|c|c|}
\hline 55 & 33 & 8.8 & 2.2 & 495.0 & 1208.9 & 44.2 \\
\hline 55 & 33 & 8.8 & 5.5 & 506.0 & 1232.0 & 42.2 \\
\hline 55 & 33 & 8.8 & 8.8 & 517.0 & 1272.7 & 40.7 \\
\hline 55 & 66 & 2.2 & 2.2 & 451.0 & 1995.4 & 61.8 \\
\hline 55 & 66 & 2.2 & 5.5 & 459.8 & 2013.0 & 60.5 \\
\hline 55 & 66 & 2.2 & 8.8 & 465.3 & 2035.0 & 59.4 \\
\hline 55 & 66 & 5.5 & 2.2 & 473.0 & 2058.1 & 58.3 \\
\hline 55 & 66 & 5.5 & 5.5 & 480.7 & 2095.5 & 57.2 \\
\hline 55 & 66 & 5.5 & 8.8 & 488.4 & 2103.2 & 56.1 \\
\hline 55 & 66 & 8.8 & 2.2 & 495.0 & 2120.8 & 54.8 \\
\hline 55 & 66 & 8.8 & 5.5 & 506.0 & 2145.0 & 47.3 \\
\hline 55 & 66 & 8.8 & 8.8 & 517.0 & 2183.5 & 51.3 \\
\hline 88 & 0 & 2.2 & 2.2 & 363.0 & 2739.0 & 79.4 \\
\hline 88 & 0 & 2.2 & 5.5 & 371.8 & 2761.0 & 78.1 \\
\hline 88 & 0 & 2.2 & 8.8 & 377.3 & 2783.0 & 77.0 \\
\hline 88 & 0 & 5.5 & 2.2 & 385.0 & 2801.7 & 75.9 \\
\hline 88 & 0 & 5.5 & 5.5 & 393.8 & 2849.0 & 76.1 \\
\hline 88 & 0 & 5.5 & 8.8 & 399.3 & 2893.0 & 76.6 \\
\hline 88 & 0 & 8.8 & 2.2 & 407.0 & 2974.4 & 76.8 \\
\hline 88 & 0 & 8.8 & 5.5 & 418.0 & 2959.0 & 71.5 \\
\hline 88 & 0 & 8.8 & 8.8 & 429.0 & 2927.1 & 68.2 \\
\hline 88 & 33 & 2.2 & 2.2 & 187.0 & 3315.4 & 104.1 \\
\hline 88 & 33 & 2.2 & 5.5 & 195.8 & 3336.3 & 102.3 \\
\hline 88 & 33 & 2.2 & 8.8 & 200.2 & 3355.0 & 101.2 \\
\hline 88 & 33 & 5.5 & 2.2 & 209.0 & 3378.1 & 100.5 \\
\hline 88 & 33 & 5.5 & 5.5 & 217.8 & 3399.0 & 99.0 \\
\hline 88 & 33 & 5.5 & 8.8 & 224.4 & 3421.0 & 97.9 \\
\hline 88 & 33 & 8.8 & 2.2 & 231.0 & 3440.8 & 97.0 \\
\hline 88 & 33 & 8.8 & 5.5 & 242.0 & 3366.0 & 95.7 \\
\hline 88 & 33 & 8.8 & 8.8 & 253.0 & 3503.5 & 93.5 \\
\hline 88 & 66 & 2.2 & 2.2 & 539.0 & 1116.5 & 58.3 \\
\hline 88 & 66 & 2.2 & 5.5 & 547.8 & 1144.0 & 56.1 \\
\hline 88 & 66 & 2.2 & 8.8 & 553.3 & 1166.0 & 55.0 \\
\hline 88 & 66 & 5.5 & 2.2 & 561.0 & 1208.9 & 53.0 \\
\hline 88 & 66 & 5.5 & 5.5 & 569.8 & 1232.0 & 50.6 \\
\hline 88 & 66 & 5.5 & 8.8 & 575.3 & 1276.0 & 48.4 \\
\hline 88 & 66 & 8.8 & 2.2 & 583.0 & 1303.5 & 47.7 \\
\hline 88 & 66 & 8.8 & 5.5 & 595.1 & 1342.0 & 44.0 \\
\hline 88 & 66 & 8.8 & 8.8 & 605.0 & 1397.0 & 42.5 \\
\hline \multicolumn{4}{|c|}{ Minimum value } & 11.0 & 1053.8 & 40.7 \\
\hline \multicolumn{4}{|c|}{ Maximum value } & 605.0 & 3503.5 & 104.1 \\
\hline \multicolumn{4}{|c|}{ The index of correlation } & 0.6849 & 0.7149 & 0.6551 \\
\hline \multicolumn{4}{|c|}{ Coefficient of determination } & 0.4611 & 0.5111 & 0.4292 \\
\hline
\end{tabular}


vector of operated variable (design data); $F(X)=\left\{F_{1}(X) F_{2}(X) I_{1}(X), I_{2}(X)\right\}$ -vector criterion (41)-(44) which everyone a component represents a vector of criteria (characteristics) of the system which functionally depend on discrete values of a vector of variables $X=\left\{x_{j}, j=\overline{1, N}\right\}$;

$F_{1}(X)=\left\{f_{k}(X), k=\overline{1, K_{1}^{\text {def }}}\right\}, \quad F_{2}(X)=\left\{f_{k}(X), k=\overline{1, K_{2}^{\text {def }}}\right\}$-a set of the $\max$ and min functions respectively;

$$
\begin{aligned}
& I_{1}(X)=\left\{\left\{f_{k}\left(X_{i}, i=\overline{1, M}\right)\right\}^{\mathrm{T}}, k=\overline{1, K_{1}^{\text {unc }}}\right\} \%, \\
& I_{2}(X)=\left\{\left\{f_{k}\left(X_{i}, i=\overline{1, M}\right)\right\}^{\mathrm{T}}, k=\overline{1, K_{2}^{\text {unc }}}\right\} \text {-set of matrixes of max and min re- }
\end{aligned}
$$

spectively; $K_{1}^{\text {def }}, K_{2}^{\text {def }}$ (definiteness), $K_{1}^{\text {unc }}, K_{2}^{\text {unc }}$ (uncertainty) the set of criteria of $\max$ and $\min$ created in the conditions of definiteness and uncertainty;

In (48) $f_{k}^{\min } \leq f_{k}(X) \leq f_{k}^{\max }, k=\overline{1, K}-$ a vector function of the restrictions imposed on functioning of technical system; $x_{j}^{\min } \leq x_{j} \leq x_{j}^{\max }, j=\overline{1, N}$ - parametrical restrictions.

It is assumed that the functions $f_{k}(X), k=\overline{1, K}$ are differentiable and convex, $g_{i}(X), i=\overline{1, M}$ are continuous, and the set of admissible points $S$ given by constraints (8) is non-empty and is a compact:

$$
\boldsymbol{S}=\left\{X \in \boldsymbol{R}^{n} \mid G(X) \leq 0, X^{\min } \leq X \leq X^{\max }\right\} \neq \varnothing .
$$

Building a model in certainty conditions;

Construction in conditions of certainty is determined by the functional dependence of each characteristic and constraints on the parameters of the technical system. In our example, characteristic (42) and constraints (41) are known. Using data (41), (42) we construct a two-criterion problem of nonlinear programming in conditions of certainty:

$$
\begin{aligned}
f_{4}(X)= & 19.253-0.0081 * x_{1}-0.7005 * x_{2}-0.3605 * x_{3}+0.9769 * x_{4} \\
& +0.0126 * x_{1} * x_{2}+0.0644 * x_{1} * x_{3}-0 * x_{1} * x_{4}+0.0396 * x_{2} * x_{3} \\
& +0.0002 * x_{2} * x_{4}+0.0004 * x_{3} * x_{4}-0.0016 * x_{1}^{2}+0.0027 * x_{2}^{2} \\
& +0.0045 * x_{3}^{2}-0.0235 * x_{4}^{2}
\end{aligned}
$$

restrictions: $22 \leq x_{1} \leq 88,0 \leq x_{2} \leq 66,2.2 \leq x_{3} \leq 8.8,2.2 \leq x_{4} \leq 8.8$

These data are used further at creation of mathematical model of technical system.

Construction in the conditions of not certainty.

Construction in the conditions of uncertainty consists in use of the qualitative and quantitative descriptions of technical system received by the principle "input-output" in Table 1. Transformation of information (basic data of $\left.y_{1}(X), y_{2}(X), y_{3}(X)\right)$ to a functional type of $f_{1}(X), f_{2}(X), f_{3}(X)$ is carried out by use of mathematical methods (the regression analysis). Basic data of Table 1 are created in MATLAB system in the form of a matrix:

$$
I=|X, Y|=\left\{x_{i 1}, x_{i 2}, x_{i 3}, x_{i 4}, y_{i 2}, y_{i 3}, i=\overline{1, M}\right\} .
$$


For each set experimental these $y_{k}, k=2,3$ function of regression on a method of the smallest squares $\min \sum_{i=1}^{M}\left(y_{i}-\overline{y_{i}}\right)^{2}$ in MATLAB. $A_{k}$-polynomial defining interrelation of the parameters.

$X_{i}=\left\{x_{i 1}, x_{i 2}, x_{i 3}, x_{i 4}\right\}$ and functions $\overline{y_{k i}}=f\left(X_{i}, A_{k}\right), k=2,3$ is for this purpose formed.

As a result of calculations we received system of coefficients of $A_{k}=\left\{A_{0 k}, A_{1 k}, \cdots, A_{14 k}\right\}$ which define coefficients of quadratic a polynomial (function):

$$
\begin{aligned}
f_{k}(X, A)= & A_{0 k}+A_{1 k} x_{1}+A_{2 k} x_{2}+A_{3 k} x_{3}+A_{4 k} x_{4}+A_{5 k} x_{1} * x_{2}+A_{6 k} x_{1} * x_{3} \\
& +A_{7 k} x_{1} * x_{4}+A_{8 k} x_{2} * x_{3}+A_{9 k} x_{2} * x_{4}+A_{10 k} x_{3} * x_{4}+A_{11 k} x_{1}^{2} \\
& +A_{12 k} x_{2}^{2}+A_{13 k} x_{3}^{2}+A_{14 k} x_{4}^{2}, \quad k=1,2,3
\end{aligned}
$$

As a result of calculations of coefficients of $A_{k}, k=3$, we received the $f_{1}(X)$, $f_{2}(X)$ and $f_{3}(X)$ function:

$$
\begin{aligned}
& f_{1}(X)= 296.85-1.874 * x_{1}-2.911 * x_{2}+8.939 * x_{3}+10.936 * x_{4} \\
&+0.0734 * x_{1} * x_{2}-0.0047 * x_{1} * x_{3}-0.0128 * x_{1} * x_{4} \\
&+0.0563 * x_{2} * x_{3}-0.0789 * x_{2} * x_{4}-0.0025 * x_{3} * x_{4} \\
&+0.0108 * x_{1}^{2}+0.0089 * x_{2}^{2}-0.1844 * x_{3}^{2}-0.3808 * x_{4}^{2} \\
& f_{2}(X)= 875.3+23.893 * x_{1}-30.866 * x_{2}-25.858 * x_{3}-45 * x_{4} \\
&-0.6984 * x_{1} * x_{2}+0.4276 * x_{1} * x_{3}+0.6793 * x_{1} * x_{4} \\
&-0.1167 * x_{2} * x_{3}+0.2969 * x_{2} * x_{4}-0.0093 * x_{3} * x_{4} \\
&+0.0362 * x_{1}^{2}+0.0331 * x_{2}^{2}+2.9158 * x_{3}^{2}+2.4052 * x_{4}^{2} \\
& f_{3}(X)=43.734+0.6598 * x_{1}+0.4493 * x_{2}-0.3094 * x_{3}-1.8334 * x_{4} \\
&-0.01 * x_{1} * x_{2}-0.0062 * x_{1} * x_{3}+0.0146 * x_{1} * x_{4} \\
&-0.013 * x_{2} * x_{3}+0.0121 * x_{2} * x_{4}-0.0004 * x_{3} * x_{4} \\
&-0.0003 * x_{1}^{2}-0.0002 * x_{2}^{2}+00.0254 * x_{3}^{2}+0.0939 * x_{4}^{2}
\end{aligned}
$$

The minimum and maximum values of experimental data $y_{1}(X), y_{2}(X), y_{3}(X)$ are presented in the lower part of Table 1 . The minimum and maximum values of the functions $f_{1}(X), f_{2}(X), f_{3}(X)$ slightly differ from experimental data. The index of correlation and coefficients of determination are presented in the lower lines of Table 1. Results of the regression analysis (54)-(55) are used further at creation of mathematical model of technical system.

Construction of a numerical model of the system under certainty and uncertainty.

For creation of numerical model of the system we used: the functions received conditions of definiteness (9) and uncertainty (53), (54), (55); parametrical restrictions (50).We considered functions (49) and (53), (54), (55) as the criteria defining focus of functioning of the system. A set of criteria $K=4$ included two criteria of $f_{1}(X), f_{3}(X) \rightarrow \max$ and two $f_{2}(X), f_{4}(X) \rightarrow \min$. As a result model of functioning of the system was presented a vector problem of mathematical programming: 


$$
\begin{aligned}
\text { opt } F(X)=\{ & \max F_{1}(X)=\left\{\max f_{1}(X) \equiv 296.85-1.874 * x_{1}-2.911 * x_{2}\right. \\
& +8.939 * x_{3}+10.936 * x_{4}+0.0734 * x_{1} * x_{2}-0.0047 * x_{1} * x_{3} \\
& -0.0128 * x_{1} * x_{4}+0.0563 * x_{2} * x_{3}-0.0789 * x_{2} * x_{4} \\
& -0.0025 * x_{3} * x_{4}+0.0108 * x_{1}^{2}+0.0089 * x_{2}^{2}-0.1844 * x_{3}^{2} \\
& -0.3808 * x_{4}^{2}, \\
\max f_{3}(X) \equiv & 43.734+0.6598 * x_{1}+0.4493 * x_{2}-0.3094 * x_{3}-1.8334 * x_{4} \\
& -0.01 * x_{1} * x_{2}-0.0062 * x_{1} * x_{3}+0.0146 * x_{1} * x_{4} \\
& -0.013 * x_{2} * x_{3}+0.0121 * x_{2} * x_{4}-0.0004 * x_{3} * x_{4} \\
& \left.-0.0003 * x_{1}^{2}-0.0002 * x_{2}^{2}+00.0254 * x_{3}^{2}+0.0939 * x_{4}^{2}\right\}, \\
\min F_{1}(X)=\{ & \min f_{2}(X) \equiv 875.3+23.893 * x_{1}-30.866 * x_{2}-25.858 * x_{3} \\
& -45 * x_{4}-0.6984 * x_{1} * x_{2}+0.4276 * x_{1} * x_{3}+0.6793 * x_{1} * x_{4} \\
& -0.1167 * x_{2} * x_{3}+0.2969 * x_{2} * x_{4}-0.0093 * x_{3} * x_{4} \\
& +0.0362 * x_{1}^{2}+0.0331 * x_{2}^{2}+2.9158 * x_{3}^{2}+2.4052 * x_{4}^{2},
\end{aligned}
$$

$\min f_{4}(X) \equiv 19.253-0.0081 * x_{1}-0.7005 * x_{2}-0.3605 * x_{3}+0.9769 * x_{4}$

$$
\begin{aligned}
& +0.0126 * x_{1} * x_{2}+0.0644 * x_{1} * x_{3}-0 * x_{1} * x_{4}+0.0396 * x_{2} * x_{3} \\
& +0.0002 * x_{2} * x_{4}+0.0004 * x_{3} * x_{4}-0.0016 * x_{1}^{2}+0.0027 * x_{2}^{2} \\
& \left.\left.+0.0045 * x_{3}^{2}-0.0235 * x_{4}^{2}\right\}\right\},
\end{aligned}
$$

restrictions: $22 \leq x_{1} \leq 88,0 \leq x_{2} \leq 66,2.2 \leq x_{3} \leq 8.8,2.2 \leq x_{4} \leq 8.8$.

The vector problem of mathematical programming (56)-(60) represents the model decision making under certainty and uncertainty in the aggregate.

Stage 3. The solution of the vector problem of mathematical programming $(V P M P)-$ model of the system at equivalent criteria.

To solve the vector problems of mathematical programming (56)-(60), methods based on the axioms of the normalization of criteria and the principle of guaranteed results are presented, which follow from axiom 1 and the principle of optimality 1.

The solution of a vector problem (56)-(60) with was submitted as sequence of steps.

Step 1. Problems (54)-(58) were solved by each criterion separately, thus used the function fmincon (...) of Matlab system, the appeal to the function fmincon (...) is considered in [12]-[17].

As a result of calculation for each criterion we received optimum points: $X_{k}^{*}$ and $f_{k}^{*}=f_{k}\left(X_{k}^{*}\right), k=\overline{1, K}$-sizes of criteria in this point, i.e. the best decision on each criterion:

$$
\begin{aligned}
& X_{1}^{*}=\left\{x_{1}=88.0, x_{2}=66.0, x_{3}=8.8, x_{4}=2.2\right\}, f_{1}^{*}=f_{1}\left(X_{1}^{*}\right)=-535.06 \\
& X_{2}^{*}=\left\{x_{1}=22.0, x_{2}=0.0, x_{3}=2.83, x_{4}=6.25\right\}, f_{2}^{*}=f_{2}\left(X_{2}^{*}\right)=1301.2 \\
& X_{3}^{*}=\left\{x_{1}=88.0, x_{2}=0.0, x_{3}=2.2, x_{4}=8.8\right\}, f_{3}^{*}=f_{3}\left(X_{3}^{*}\right)=-100.15 \\
& X_{4}^{*}=\left\{x_{1}=22.0, x_{2}=62.17, x_{3}=2.2, x_{4}=2.2\right\}, f_{4}^{*}=f_{4}\left(X_{4}^{*}\right)=12.247
\end{aligned}
$$


Restrictions (58) and points of an optimum $X_{1}^{*}, X_{2}^{*}, X_{3}^{*}, X_{4}^{*}$ in coordinates $\left\{X_{1}, X_{2}\right\}$ are presented on Figure 1.

Step 2. We defined the worst unchangeable part of each criterion (anti-optimum):

$$
\begin{gathered}
X_{1}^{0}=\left\{x_{1}=22.0, x_{2}=66.0, x_{3}=2.2, x_{4}=2.2\right\}, f_{1}^{0}=f_{1}\left(X_{1}^{0}\right)=243.25 ; \\
X_{2}^{0}=\left\{x_{1}=88.0, x_{2}=0.0, x_{3}=8.8, x_{4}=8.8\right\}, f_{2}^{0}=f_{2}\left(X_{2}^{0}\right)=-3903.1 ; \\
X_{3}^{0}=\left\{x_{1}=22.0, x_{2}=0.0, x_{3}=8.8, x_{4}=8.07\right\}, f_{3}^{0}=\left(X_{3}^{0}\right)=50.03 ; \\
X_{4}^{0}=\left\{x_{1}=88.0, x_{2}=66.0, x_{3}=8.8, x_{4}=8.8\right\}, f_{4}^{0}=f_{4}\left(X_{4}^{0}\right)=-121.83 .
\end{gathered}
$$

Step 3. Performed system analysis of a set of points, optimum across Pareto, (i.e. the analysis for each criterion). In points of an optimum of $X^{*}=\left\{X_{1}^{*}, X_{2}^{*}, X_{3}^{*}, X_{4}^{*}\right\}$ sizes of criterion functions of $F\left(X^{*}\right)=\left\|f_{q}\left(X_{k}^{*}\right)\right\|_{q=\overline{1, K}}^{k=\overline{1, K}}$ determined. Calculated a vector of $F=\left(d_{1} d_{2} d_{3} d_{4}\right)^{\mathrm{T}}$-deviations by each criterion on an admissible set of $S: \quad d_{k}=f_{k}^{*}-f_{k}^{0}, k=\overline{1,4}$, and matrix of relative estimates of

$$
\begin{gathered}
d_{k}=f_{k}^{*}-f_{k}^{0}, k=\overline{1,4} \text {, where } \lambda_{k}(X)=\left(f_{k}^{*}-f_{k}^{0}\right) / d_{k} . \\
F\left(X^{*}\right)=\left\|\begin{array}{cccc}
535.1 & 1731.9 & 58.1 & 117.0 \\
317.6 & 1301.2 & 51.3 & 26.5 \\
192.5 & 3614.3 & 100.2 & 24.6 \\
244.0 & 2458.2 & 67.7 & 12.2
\end{array}\right\|, d_{k}=\left\|\begin{array}{c}
291.8 \\
-2602.0 \\
50.12 \\
-109.58
\end{array}\right\|, \\
\lambda\left(X^{*}\right)=\left\|\begin{array}{cccc}
1.0000 & 0.8345 & 0.1603 & 0.0443 \\
0.2548 & 1.0000 & 0.0244 & 0.8697 \\
-0.1740 & 0.1110 & 1.0000 & 0.8870 \\
0.0027 & 0.5553 & 0.3532 & 1.0000
\end{array}\right\| .
\end{gathered}
$$

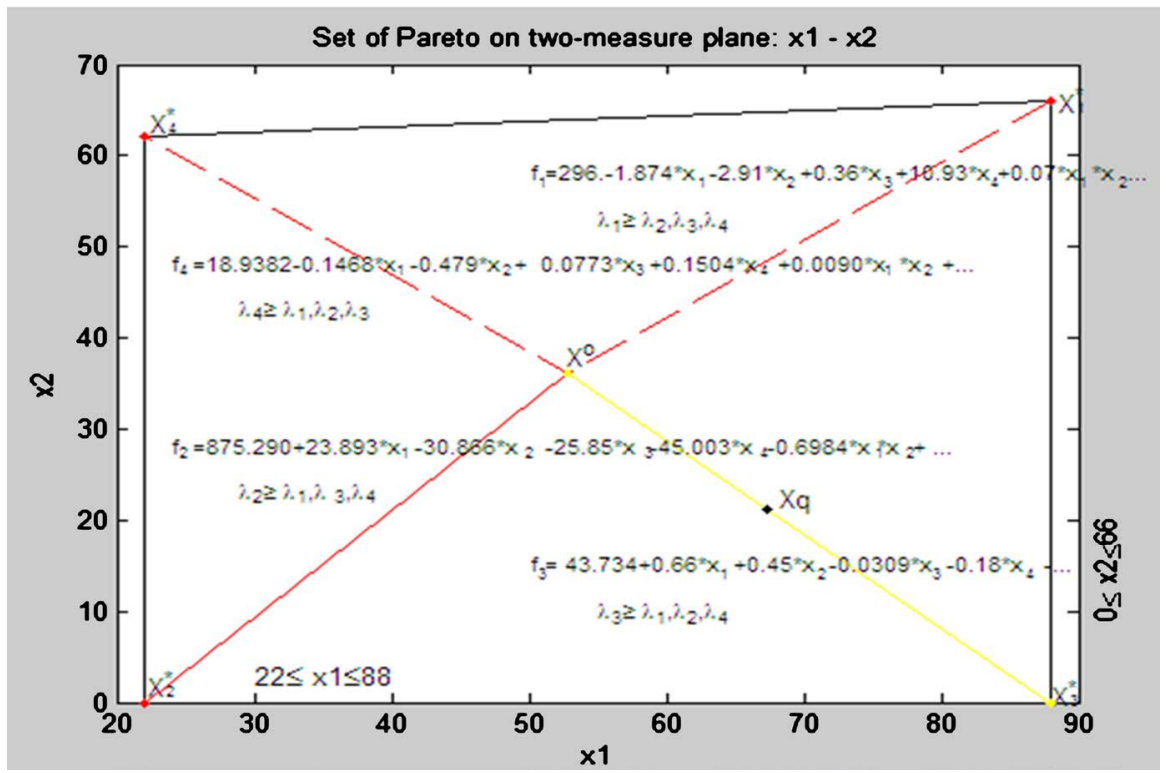

Figure 1. Pareto's great number, $S^{o} \subset S, X_{1}^{*}, X_{2}^{*}, X_{3}^{*}, X_{4}^{*}$ in two-dimensional system of coordinates $\left\{x_{1}, x_{2}\right\}$. 
The analysis of sizes of criteria in relative estimates showed that in points of an optimum of $X^{*}=\left\{X_{1}^{*}, X_{2}^{*}, X_{3}^{*}, X_{4}^{*}\right\}$ the relative assessment is equal to unit. Other criteria there is much less than unit. It is required to find such point (parameters) at which relative estimates are closest to unit. The steps 4, 5 are directed on the solution of this problem.

Step 4. Creation of $\lambda$-problem is carried out in two stages: originally the maximine problem ofoptimization with the normalized criteria is under construction:

$$
\lambda^{o}=\max _{X \in S} \min _{k \in K} \lambda_{k}(X), G(X) \leq 0, X \geq 0,
$$

which at the second stage was transformed to a standard problem of mathematical programming $(\lambda$-problem):

$$
\lambda^{o}=\max \lambda,
$$

$$
\text { at restrictions } \lambda-\frac{f_{1}(X)-f_{1}^{0}}{f_{1}^{*}-f_{1}^{0}} \leq 0 \text {, }
$$

$$
\begin{gathered}
\lambda-\frac{f_{3}(X)-f_{3}^{0}}{f_{3}^{*}-f_{3}^{0}} \leq 0, \\
\lambda-\frac{f_{2}(X)-f_{2}^{0}}{f_{2}^{*}-f_{2}^{0}} \leq 0, \\
\lambda-\frac{f_{4}(X)-f_{4}^{0}}{f_{4}^{*}-f_{4}^{0}} \leq 0,
\end{gathered}
$$

$$
0 \leq \lambda \leq 1,22 \leq x_{1} \leq 88,0 \leq x_{2} \leq 66,2.2 \leq x_{3} \leq 8.8,2.2 \leq x_{4} \leq 8.8,
$$

where the vector of unknown had dimension of $N+1: \quad X=\left\{x_{1}, \cdots, x_{N}, \lambda\right\}$; the functions $f_{1}(X), f_{2}(X), f_{3}(X), f_{4}(X)$ correspond (64)-(68) respectively. Substituting the numerical values of the functions $f_{1}(X), f_{2}(X), f_{3}(X), f_{4}(X)$, we get the $\lambda$-problem of the following form:

$$
\lambda^{0}=\max \lambda,
$$

at restrictions $\lambda-\frac{296.8-1.874 * x_{1}-2.91 * x_{2}-\cdots-0.184 * x_{3}^{2}-0.38 * x_{4}^{2}-f_{1}^{0}}{f_{1}^{*}-f_{1}^{0}} \leq 0$,

$$
\begin{gathered}
\lambda-\frac{43.734+0.6598 * x_{1}+0.449 * x_{2}-\cdots+0.0254 * x_{3}^{2}+0.0939 * x_{4}^{2}-f_{3}^{0}}{f_{3}^{*}-f_{3}^{0}} \leq 0, \\
\lambda-\frac{875.3+23.9 * x_{1}-30.8 * x_{2}-\cdots+2.9158 * x_{3}^{2}+2.4052 * x_{4}^{2}-f_{2}^{0}}{f_{2}^{*}-f_{2}^{0}} \leq 0, \\
\lambda-\frac{19.253-0.0081 * x_{1}-0.7005 * x_{2}-\cdots+0.0045 * x_{3}^{2}-0.0235 * x_{4}^{2}-f_{4}^{0}}{f_{4}^{*}-f_{4}^{0}} \leq 0, \\
0 \leq \lambda \leq 1,22 \leq x_{1} \leq 88,0 \leq x_{2} \leq 66,2.2 \leq x_{3} \leq 8.8,2.2 \leq x_{4} \leq 8.8,
\end{gathered}
$$

Appeal to function fmincon $(\ldots)$ :

$[\mathrm{Xo}, \mathrm{Lo}]=$ fmincon ('Z_TehnSist_4Krit_L', X0, Ao, bo, Aeq, beq, lbo, ubo, 'Z_TehnSist_LConst', options). 
As a result of the solution of a vector problem of mathematical programming (56)-(60) at equivalent criteria and $\lambda$-problem corresponding to it (69)-(74) received:

$\boldsymbol{X}^{o}=\left\{X^{o}, \lambda^{o}\right\}=\left\{X^{o}=\left\{x_{1}=52.9, x_{2}=36.097, x_{3}=8.8, x_{4}=2.2\right\}, \lambda^{o}=0.3179\right\}$,

an optimum point-design data of the system, point $\boldsymbol{X}^{\boldsymbol{0}}$ is presented in Figure 1;

$f_{k}\left(X^{o}\right), k=\overline{1, K}$ - sizes of criteria (characteristics of technical system):

$\left\{f_{1}\left(X^{o}\right)=336.0, f_{2}\left(X^{o}\right)=2239.5, f_{3}\left(X^{o}\right)=65.962, f_{4}\left(X^{o}\right)=58.435\right\} ;(76$ $\lambda_{k}\left(X^{o}\right), k=\overline{1, K}$ - sizes of relative estimates:

$\left\{\lambda_{1}\left(X^{o}\right)=0.3179, \lambda_{2}\left(X^{o}\right)=0.6394, \lambda_{3}\left(X^{o}\right)=0.3179, \lambda_{4}\left(X^{o}\right)=0.5785\right\} ;$

$\lambda^{\circ}=0.3179$ is the maximum lower level among all relative estimates measured in relative units:

$$
\lambda^{o}=\min \left(\lambda_{1}\left(X^{o}\right), \lambda_{2}\left(X^{o}\right), \lambda_{3}\left(X^{o}\right), \lambda_{4}\left(X^{o}\right)\right)=0.3179 .
$$

A relative assessment $-\lambda^{\circ}$ call the guaranteed result in relative units, i.e. $\lambda_{k}\left(X^{o}\right)$ and according to the characteristic of technical $f_{k}\left(X^{o}\right)$ system it is impossible to improve, without worsening thus other characteristics.

We will notice that according to Theorem 1 , in $\boldsymbol{X}^{\boldsymbol{b}}$ point criteria 1,3 are contradictory. This contradiction is defined by equality of $\lambda_{1}\left(X^{o}\right)=\lambda_{3}\left(X^{\circ}\right)=\lambda^{\circ}=0.3179$, and other criteria an inequality of $\left\{\lambda_{2}\left(X^{o}\right)=0.6394, \lambda_{4}\left(X^{o}\right)=0.5785\right\}>\lambda^{o}$.

Thus, Theorem 1 forms a basis for determination of correctness of the solution of a vector problem. In a vector problem of mathematical programming, as a rule, for two criteria equality is carried out:

$$
\lambda^{o}=\lambda_{q}\left(X^{o}\right)=\lambda_{p}\left(X^{o}\right), q, p \in \boldsymbol{K}, X \in S,
$$

and for other criteria is defined as an inequality: $\lambda^{o} \leq \lambda_{k}\left(X^{o}\right), \forall k \in K, q \neq p \neq k$.

Stage 4. Creation of geometrical interpretation of results of the decision in a three-dimensional coordinate system in relative units.

In an admissible set of points of $S$ formed by restrictions (74), optimum points $X_{1}^{*}, X_{2}^{*}, X_{3}^{*}, X_{4}^{*}$ united in a contour, presented a set of points, optimum across Pareto, to $S^{o} \subset S$, Figure 1 .

Coordinates of these points, and also characteristics of technical system in relative units of $\lambda_{1}(X), \lambda_{2}(X), \lambda_{3}(X), \lambda_{4}(X)$ are shown in Figure 2 in three measured space, where the third axis of $\lambda$-a relative assessment.

Discussion. Looking at a Figure 2, we can provide changes of all functions of $\lambda_{1}(X), \lambda_{2}(X), \lambda_{3}(X), \lambda_{4}(X)$ in four measured space. We will consider, for example, an optimum point of $X_{3}^{*}$. The $\lambda_{3}(X)$ function is created from the functions $f_{3}(X)$ with variable coordinates $\left\{X_{1}, X_{2}\right\}$ and with constant coordinates $\left\{x_{3}=8.8, x_{4}=2.2\right\}$, taken from an optimum point $X^{\circ}(75)$. In a point $X_{3}^{*}$ the relative assessment of $\lambda_{3}\left(X_{3}^{*}\right)=0.83$-is shown in Figure 2 by a black point. 


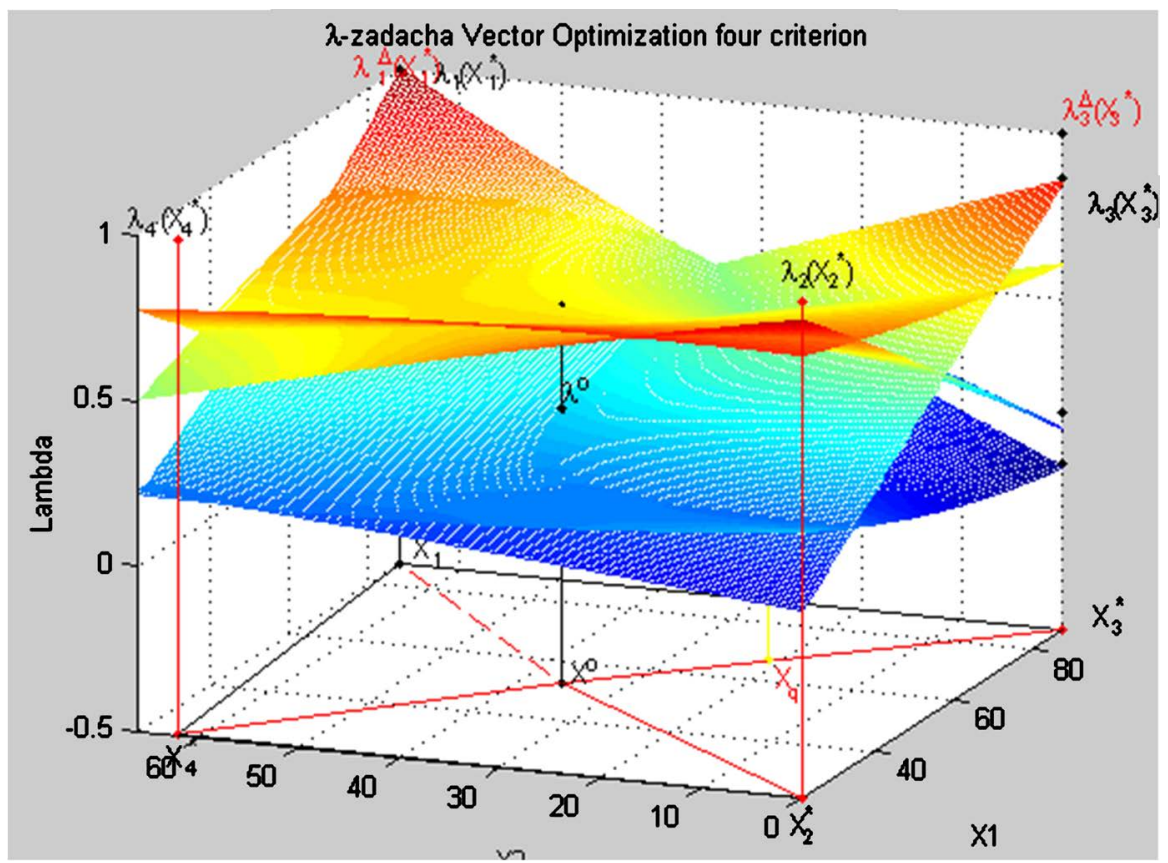

Figure 2. The solution of $\lambda$-problem in three-dimensional system of coordinates of $x_{1}, x_{2}$ and $\lambda$.

But we know that the relative assessment of $\lambda_{3}\left(X_{3}^{*}\right)$ received from the $f_{3}\left(X_{3}^{*}\right)$ function on the third step is equal to unit, we will designate it as $\lambda_{3}^{\Delta}\left(X_{3}^{*}\right)=1-$ is shown in Figure 2 by a red point. The difference between $\lambda_{3}^{\Delta}\left(X_{3}^{*}\right)=1$ and $\lambda_{3}\left(X_{3}^{*}\right)=0.83$ is an error $\Delta=0.17$ transitions from four measured (and generally $N$-dimensional) to two-dimensional area.

The point $X_{1}^{*}$ and appropriate relative estimates of $\lambda_{1}\left(X_{1}^{*}\right)$ and $\lambda_{1}^{\Delta}\left(X_{1}^{*}\right)$ is similarly shown.

Thus, for the first time in domestic and foreign practice transition and its geometrical illustration from $\mathrm{N}$-dimensional to two-dimensional measurement of function is shown in vector problems of mathematical programming with the appropriate errors.

Stage 5. The solution of a vector problem of mathematical programmingmodel of the system at the given priority of the criterion.

The decision maker is usually the system designer.

Step 1. We solve a vector problem with equivalent criteria. The algorithm of the decision is presented in Stage 3. Numerical results of the solution of the vector problem are given above.

Pareto's great number of $S^{o} \subset S$ lies between optimum points $X_{1}^{*} X^{o} X_{3}^{*} X^{o} X_{4}^{*} X^{o} X_{2}^{*} X^{o} X_{1}^{*}$.

We will carry out the analysis of a great number of Pareto $S^{o} \subset S$. For this purpose we will connect auxiliary points: $X_{1}^{*} X_{3}^{*} X_{4}^{*} X_{2}^{*} X_{1}^{*}$ with a point $X^{o}$ which conditionally represents the center of a great number of Pareto. As a result have received four subsets of points $X \in \boldsymbol{S}_{q}^{o} \subset \boldsymbol{S}^{o} \subset \boldsymbol{S}, q=\overline{1,4}$. The subset of $\boldsymbol{S}_{1}^{o} \subset \boldsymbol{S}^{o} \subset \boldsymbol{S}$ is characterized by the fact that the relative assessment of $\lambda_{1} \geq \lambda_{2}, \lambda_{3}, \lambda_{4}$, i.e. 
in the field of $S_{1}^{o}$ first criterion has a priority over the others. Similar to $S_{2}^{o}, S_{3}^{o}, S_{4}^{o}$-subsets of points where the second, third and fourth criterion has a priority over the others respectively. Set of points, optimum across Pareto we will designate $\boldsymbol{S}^{o}=\boldsymbol{S}_{1}^{o} \cup \boldsymbol{S}_{2}^{o} \cup \boldsymbol{S}_{3}^{o} \cup \boldsymbol{S}_{4}^{o}$. Coordinates of all received points and relative estimates are presented in two-dimensional space $\left\{x_{1}, x_{2}\right\}$ in Figure 1. These coordinates are shown in three measured space $\left\{x_{1}, x_{2}, \lambda\right\}$ in Figure 2 where the third axis of $\lambda$-a relative assessment. Restrictions of a set of points, optimum across Pareto, in Figure 14 it is lowered to -0.5 (that restrictions were visible). This information is also a basis for further research of structure of a great number of Pareto. The person making decisions, as a rule, is the designer of the system. If results of the solution of a vector problem with equivalent criteria don't satisfy the person making the decision, then the choice of the optimal solution is carried out from any subset of points of $\boldsymbol{S}_{1}^{o}, \boldsymbol{S}_{2}^{o}, \boldsymbol{S}_{3}^{o}, \boldsymbol{S}_{4}^{o}$. These subsets of Pareto points shown in Figure 8 in the form of functions

$$
f_{1}(X), f_{2}(X), f_{3}(X), f_{4}(X) \text {. }
$$

Step 2. Choice of priority criterion of $q \in \boldsymbol{K}$. From the theory (see Theorem 1 ) it is known that in an optimum point of $X^{o}$ always there are two most inconsistent criteria, $q \in \boldsymbol{K}$ and $p \in \boldsymbol{K}$ for which in relative units exact equality is carried out: $\lambda^{o}=\lambda_{q}\left(X^{o}\right)=\lambda_{p}\left(X^{o}\right), q, p \in \boldsymbol{K}, X \in \boldsymbol{S}$, and for the others it is carried out inequalities:

$$
\lambda^{o} \leq \lambda_{k}\left(X^{o}\right), \forall k \in K, q \neq p \neq k .
$$

In model of the system (54)-(58) and the corresponding $\lambda$-problem (67)-(71) such criteria are the first and third:

$$
\lambda^{o}=\lambda_{1}\left(X^{o}\right)=\lambda_{3}\left(X^{o}\right)=0.3179 .
$$

We will show the $\lambda_{1}(X)$ and $\lambda_{3}(X)$ functions separately in Figure 3 from an optimum point of

$$
\boldsymbol{X}^{o}=\left\{X^{o}, \lambda^{o}\right\} \text {. }
$$

Here all points and data about which it was told in Figure 2 are shown.

As a rule, the criterion which the decision-maker would like to improve gets out of couple of contradictory criteria. Such criterion is called "priority criterion", we will designate it $q=3 \in \boldsymbol{K}$. This criterion is investigated in interaction with the first criterion of $q=1 \in \boldsymbol{K}$. We will allocate these two criteria from all set of the criteria $K=4$ shown in Figure 3.

On the display the message is given:

$q=$ input ('Enter priority criterion (number) of $q=$ ")-Have entered: $q=3$.

Step 3. Numerical limits of change of size of a priority of criterion of $q=3 \in \boldsymbol{K}$ are defined.

For priority criterion of $q=3$ numerical limits in physical units upon transition from a point of an optimum of $X^{0}(91)$ to the point of $X_{q}^{*}$ received on the first step are defined.

Information about the criteria for $q=3$ are given on the screen:

$$
f_{q}\left(X^{\circ}\right)=65.96 \leq f_{q}(X) \leq 100.15=f_{q}\left(X_{q}^{*}\right), q \in \boldsymbol{K} .
$$




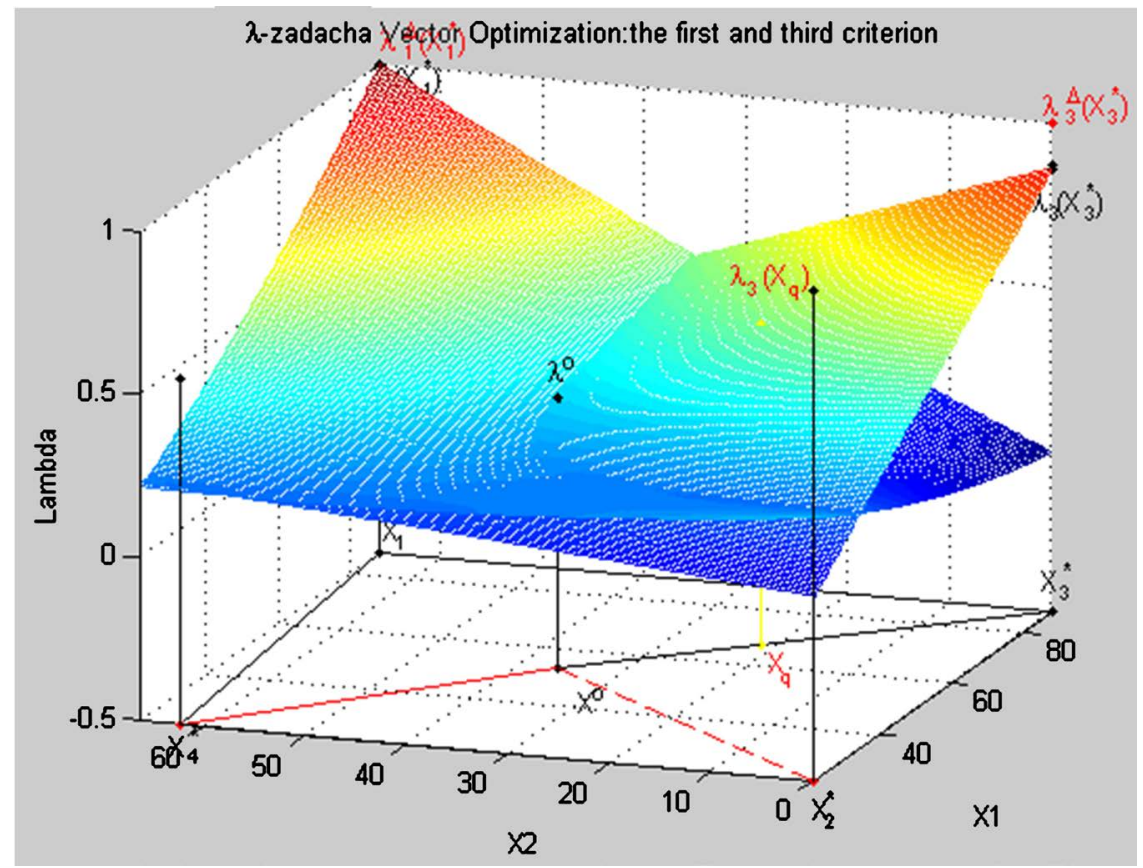

Figure 3. The solution of $\lambda$-problem (1, 3 criterion) in three-dimensional system of coordinates of $x_{1}, x_{2}$ and $\lambda$.

In relative units the criterion of $q=2$ changes in the following limits:

$$
\lambda_{q}\left(X^{\circ}\right)=0.3179 \leq \lambda_{q}(X) \leq 1=\lambda_{q}\left(X_{q}^{*}\right), q=3 \in \boldsymbol{K} .
$$

These data it is analyzed.

Step 4. Choice of size of priority criterion. $q \in \boldsymbol{K}$. (Decision-making).

The message is displayed: "Enter the size of priority criterion $f_{q}=$ "-we enter, for example, $f_{q}=80$.

Step 5. Calculation of a relative assessment.

For the chosen size of priority criterion of $f_{q}=80$ the relative assessment is calculated:

$$
\lambda_{q}=\frac{f_{q}-f_{q}^{0}}{f_{q}^{*}-f_{q}^{0}}=\frac{80-50.03}{10.15-50.03}=0.5979,
$$

which upon transition from $X^{o}$ point to $X_{q}^{*}$ according to (78) lies in limits:

$$
0.3179=\lambda_{3}\left(X^{o}\right) \leq \lambda_{3}=0.5979 \leq \lambda_{3}\left(X_{3}^{*}\right)=1, q \in \boldsymbol{K} .
$$

Step 6. Calculation of coefficient of linear approximation.

Assuming linear nature of change of criterion of $f_{q}(X)$ in (79) and according to a relative assessment of $\lambda_{q}(X)$ in (80), using standard methods of linear approximation, we will calculate proportionality coefficient between $\lambda_{q}\left(X^{0}\right), \lambda_{q^{\prime}}$, which we will call $\rho$ :

$$
\rho=\frac{\lambda_{q}-\lambda_{q}\left(X^{o}\right)}{\lambda_{q}\left(X_{q}^{*}\right)-\lambda_{q}\left(X^{o}\right)}=\frac{0.5979-0.3179}{1-0.3179}=0.4106, q=3 \in \boldsymbol{K} .
$$

Step 7. Calculation of coordinates of priority criterion with the size $f_{q}$. 
Assuming linear nature of change of a vector of $X^{q}=\left\{x_{1} x_{2}\right\}, q=3$ we will determine coordinates of a point of priority criterion with the size $f_{q}$ with a relative assessment (80):

$$
x_{\lambda=0.81}^{q=3}=\left\{x_{1}=X^{o}(1)+\rho\left(X_{q}^{*}(1)-X^{o}(1)\right), x_{2}=X^{o}(2)+\rho\left(X_{q}^{*}(2)-X^{o}(2)\right)\right\},
$$

where $X^{o}=\left\{X^{o}(1)=80.0, X^{o}(2)=69.11\right\}, X_{3}^{*}=\left\{X_{3}^{*}(1)=80.0, X_{3}^{*}(2)=0.0\right\}$.

As a result of calculations we have received point coordinates: $X^{q}=\left\{x_{1}=67.31, x_{2}=21.27\right\}$.

\section{Step 8. Calculation of the main indicators of a point of $X_{q}$.}

For the received $X_{q}$ point, we will calculate:

all criteria in physical units $f_{k}\left(X^{q}\right)=\left\{f_{k}\left(X^{q}\right), k=\overline{1, K}\right\}$ :

$$
f\left(X^{q}\right)=\left\{f_{1}\left(X^{q}\right)=313.45, f_{2}\left(X^{q}\right)=2575.7, f_{3}\left(X^{q}\right)=74.2, f_{4}\left(X^{q}\right)=60.6\right\} ;
$$

all relative estimates of criteria $\lambda^{q}=\left\{\lambda_{k}^{q}, k=\overline{1, K}\right\}$,

$$
\begin{aligned}
& \lambda_{k}\left(X^{q}\right)=\frac{f_{k}\left(X^{q}\right)-f_{k}^{0}}{f_{k}^{*}-f_{k}^{0}}, k=1, \overline{1}_{*}: \\
& \lambda_{k}\left(X^{q}\right)=\left\{\begin{aligned}
\lambda_{1}\left(X^{q}\right) & =0.2405, \lambda_{2}\left(X^{q}\right)=0.5102, \\
& \left.\lambda_{3}\left(X^{q}\right)=0.4825, \lambda_{4}\left(X^{q}\right)=0.5586\right\}
\end{aligned}\right.
\end{aligned}
$$

minimum relative assessment:

$\operatorname{minLXq}=\min (\mathrm{LXq}): \operatorname{minLXq}=\min \left(\lambda_{k}\left(X^{q}\right)\right)=0.2405 ;$

vector of priorities $P^{q}(X)=\left\{p_{k}^{q}=\frac{\lambda_{q}\left(X^{q}\right)}{\lambda_{k}\left(X^{q}\right)}, k=\overline{1, K}\right\}$ :

$$
P^{q}=\left[p_{1}^{3}=2.0061, p_{2}^{3}=0.9458, p_{3}^{3}=1.0, p_{4}^{3}=0.8637\right] ;
$$

relative assessment taking into account a criterion priority:

$$
\begin{aligned}
\lambda_{k}\left(X^{q}\right) * P^{q}=\left\{p_{1}^{3} * \lambda_{1}\left(X^{q}\right)\right. & =0.4825, p_{2}^{3} * \lambda_{2}\left(X^{q}\right)=0.4825 \\
& \left.p_{3}^{3} * \lambda_{3}\left(X^{q}\right)=0.4825, p_{4}^{3} * \lambda_{4}\left(X^{q}\right)=0.4825\right\}
\end{aligned}
$$

the minimum relative assessment taking into account a criterion priority:

$$
\lambda^{o o}=\min \left(p_{1}^{3} \lambda_{1}\left(X^{q}\right), p_{2}^{3} \lambda_{2}\left(X^{q}\right), p_{3}^{3} \lambda_{3}\left(X^{q}\right), p_{4}^{3} \lambda_{4}\left(X^{q}\right)=0.4825\right)
$$

Any point from Pareto's set $\boldsymbol{X}_{t}^{o}=\left\{\lambda_{t}^{o}, X_{t}^{o}\right\} \in \boldsymbol{S}^{o}$ can be similarly calculated.

Analysis of results. The calculated size of criterion $f_{q}\left(X_{t}^{o}\right), q \in \boldsymbol{K}$ is usually not equal to the set $f_{q}$. The error of the choice of $\Delta f_{q}=\left|f_{q}\left(X_{t}^{o}\right)-f_{q}\right|=|74.2-80|=5.8$ is defined by an error of linear approximation, $\Delta f_{q \%}=7.25 \%$.

If error $\Delta f_{q}=\left|f_{q}\left(X_{t}^{o}\right)-f_{q}\right|=|74.2-80|=5.8$, measured in physical units or as a percentage $\Delta f_{q \%}=\frac{\Delta f_{q}}{f_{q}} * 100=7.25 \%$, is more than set $\Delta f, \Delta f_{q}>\Delta f$; we pass to a step 2, if $\Delta f_{q} \leq \Delta f$, calculations come to the end. 
In the course of modeling parametrical restrictions (74) can be changed, i.e. some set of optimum decisions is received. Choose a final version which in our example included from this set of optimum decisions: parameters of technical system

$$
\boldsymbol{X}^{o}=\left\{X^{o}, \lambda^{o}\right\}=\left\{X^{o}=\left\{x_{1}=52.9, x_{2}=36.097, x_{3}=8.8, x_{4}=2.2\right\}, \lambda^{o}=0.3179\right\} ;
$$

the parameters of the technical system at a given priority criterion $q=2$ :

$$
X^{q}=\left\{x_{1}=67.31, x_{2}=21.27\right\} .
$$

Stage 6. Geometrical interpretation of results of the decision in a threedimensional coordinate system in physical units.

We introduced the parameters:

$$
\boldsymbol{X}^{o}=\left\{X^{o}, \lambda^{o}\right\}=\left\{X^{o}=\left\{x_{1}=52.9, x_{2}=36.097, x_{3}=8.8, x_{4}=2.2\right\}, \lambda^{o}=0.3179\right\} ;
$$

in the two-dimensional coordinate system $X_{1}, x_{2}$ on Figure 1, three-dimensional coordinate system $x_{1}, x_{2}$ and $\lambda$ in Figure 2. We also present these parameters in physical units for each technical system characteristic (criterion):

$$
f_{1}(X), f_{2}(X), f_{3}(X), f_{4}(X) \text {. }
$$

The first characteristic of technical system $f_{1}(X)$ in $X_{1}, X_{2}$ coordinates is shown in Figure 4. Similarly same characteristic in relative units of $\lambda_{1}(X)$ is shown in Figure 5.

Indicators $f_{1}^{\Delta}\left(X_{1}^{*}\right), f_{1}^{\Delta}\left(X_{1}^{o}\right)$ of the first of characteristics of the system (are highlighted in red color) define transition errors from four-dimensional $X=\left\{x_{1}, x_{2}, x_{3}, x_{4}\right\}$ to two-dimensional $X^{o}=\left\{x_{1}, x_{2}\right\}$ to system of coordinates.

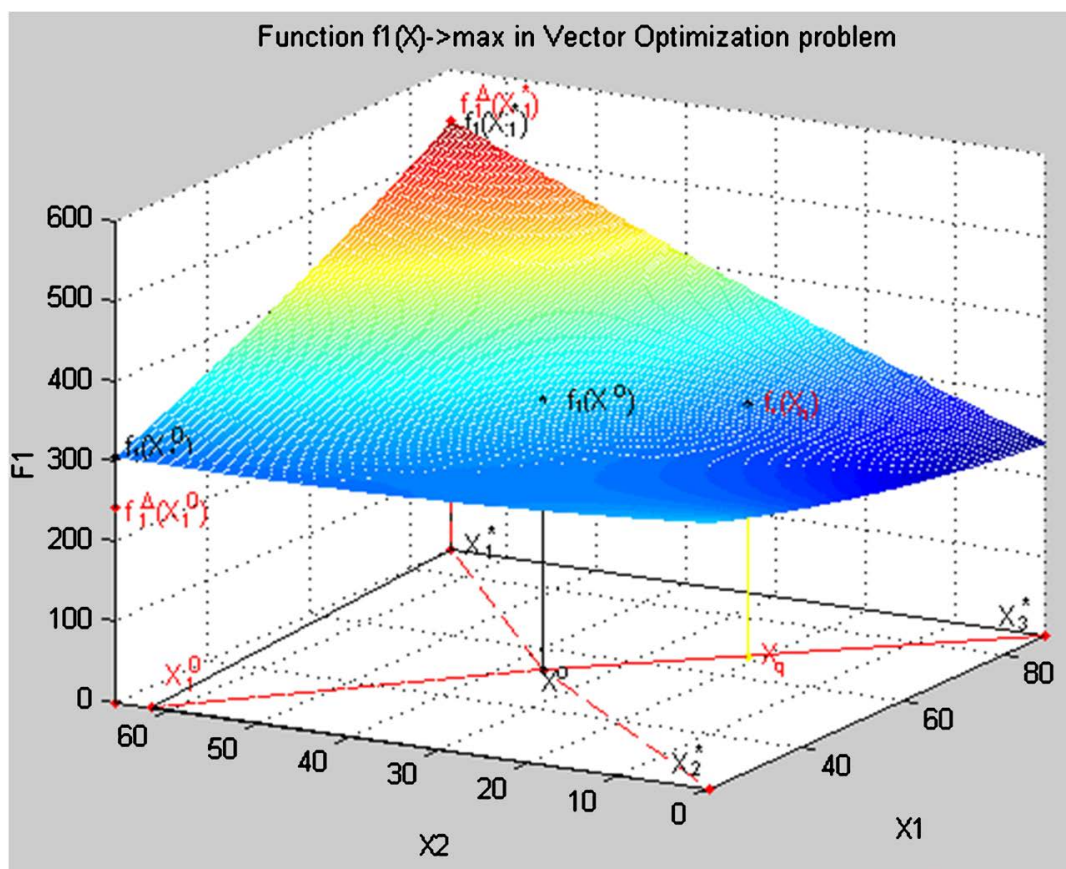

Figure 4. The first characteristics $f_{1}(X)$ in coordinates $x_{1}, x_{2}$ technical system in physical terms. 
The second characteristic of technical system $f_{2}(X)$ in $x_{1}, x_{2}$ coordinates is shown in Figure 6. Similarly same characteristic in relative units of $\lambda_{2}(X)$ is shown in Figure 7.

Indicators of the second $f_{2}^{\Delta}\left(X_{2}^{*}\right), f_{2}^{\Delta}\left(X_{2}^{o}\right)$ of characteristics of the system (are highlighted in red color) define transition errors from four-dimensional $X^{0}=\left\{x_{1}, x_{2}, x_{3}, x_{4}\right\}$ to two-dimensional $X^{0}=\left\{x_{1}, x_{2}\right\}$ to system of coordinates.

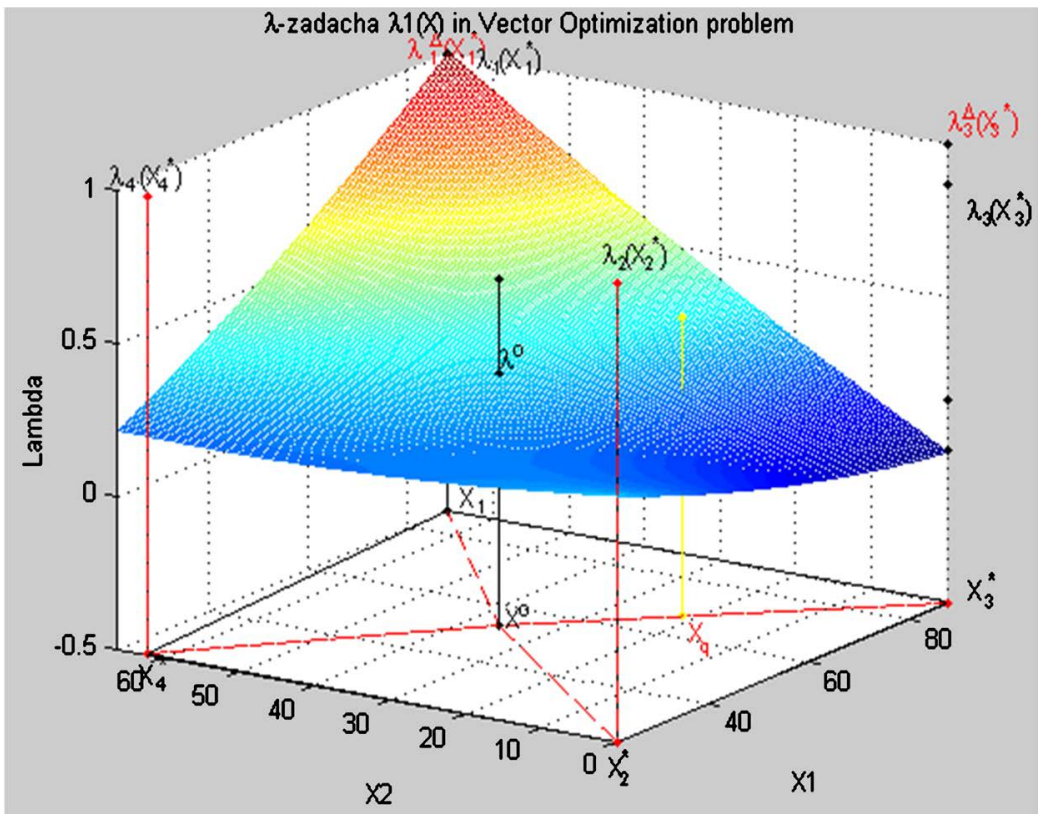

Figure 5. The first characteristics $\lambda_{1}(X)$ in coordinates $X_{1}, x_{2}$ technical system in the relative estimates.

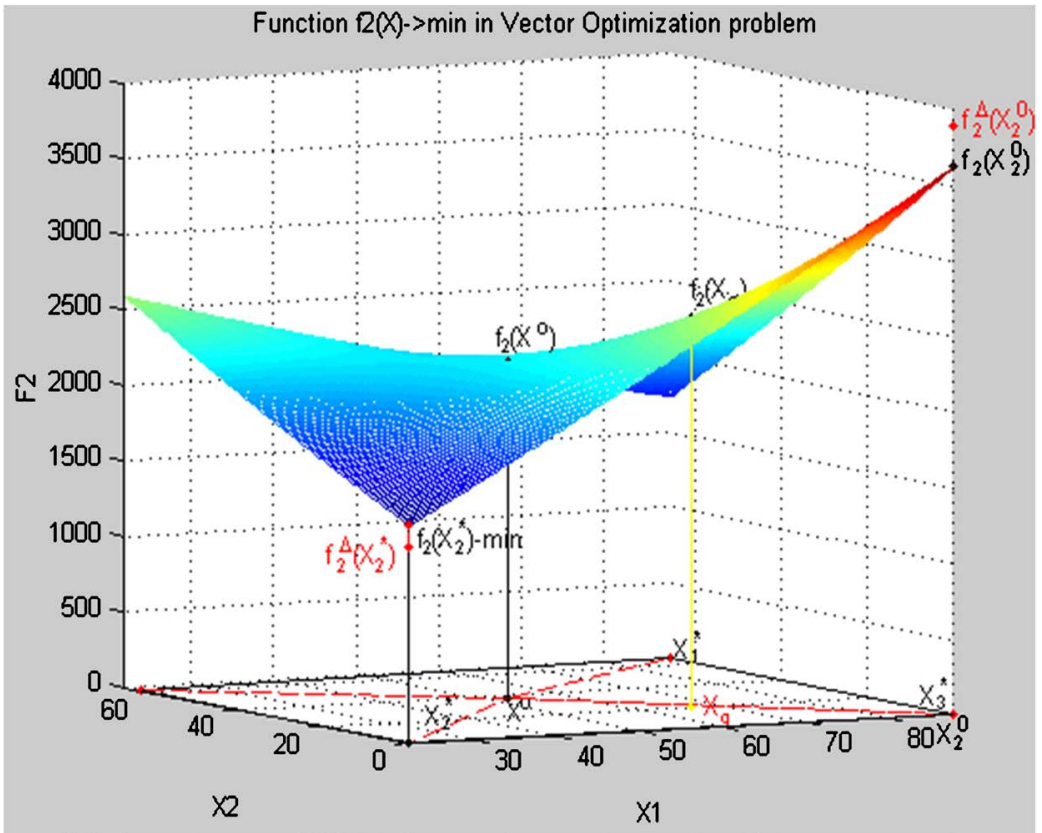

Figure 6. The second characteristics $f_{2}(X)$ in coordinates $x_{1}, x_{2}$ technical system in physical terms. 
The third characteristic of technical system $f_{3}(X)$ in $X_{1}, X_{2}$ coordinates is shown in Figure 8. Similarly same characteristic in relative units of $\lambda_{3}(X)$ is shown in Figure 9.

Indicators of the third $f_{3}^{\Delta}\left(X_{3}^{*}\right), f_{3}^{\Delta}\left(X_{3}^{o}\right)$ of characteristics of the system (are highlighted in red color) define transition errors from four-dimensional $X^{o}=\left\{x_{1}, x_{2}, x_{3}, x_{4}\right\}$ to two-dimensional $X^{o}=\left\{x_{1}, x_{2}\right\}$ to system of coordinates.

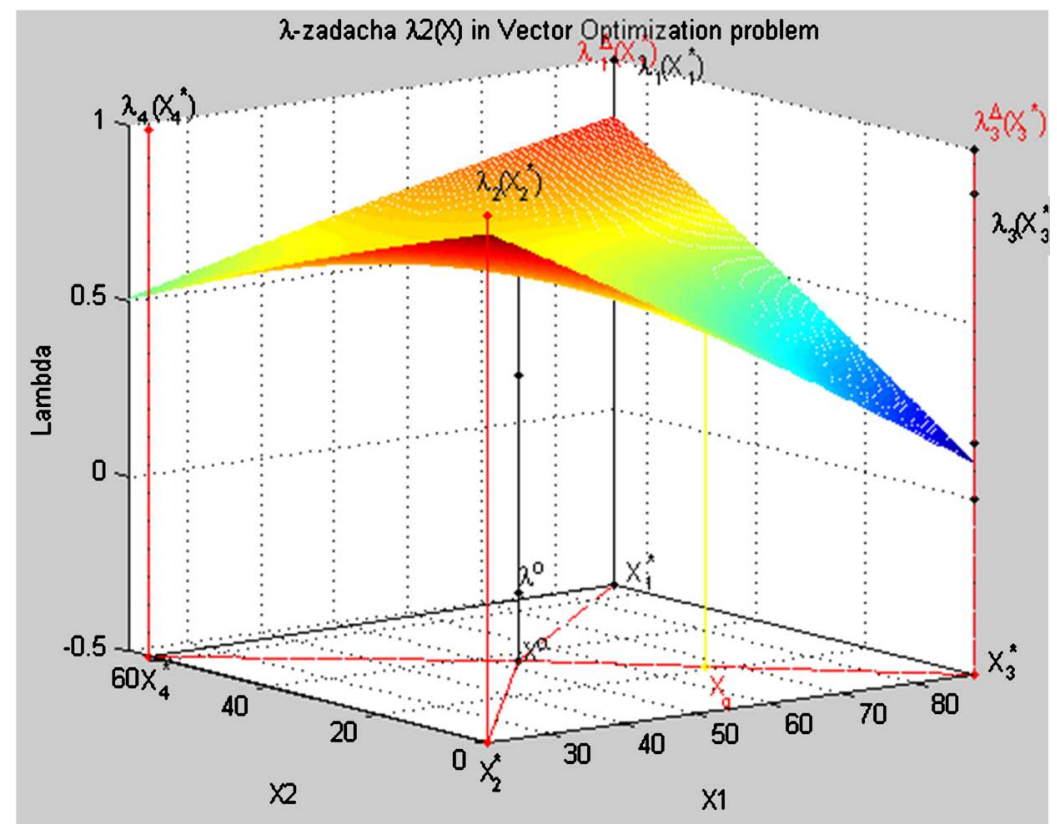

Figure 7. The second characteristics $\lambda_{2}(X)$ in coordinates $x_{1}, x_{2}$ technical system in the relative estimates.

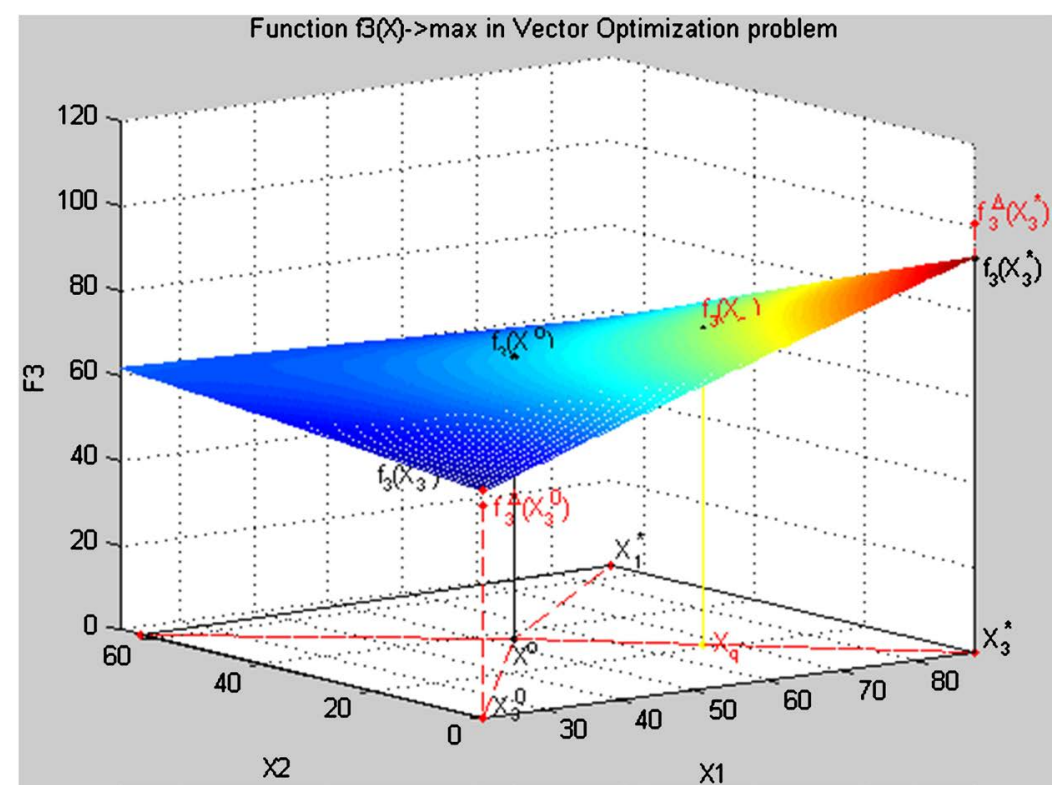

Figure 8. The third characteristics $f_{3}(X)$ in coordinates $x_{1}, x_{2}$ technical system in physical terms. 
The fourth characteristic of technical system $f_{4}(X)$ in $X_{1}, x_{2}$ coordinates is shown in Figure 10. Similarly same characteristic in relative units of $\lambda_{4}(X)$ is shown in Figure 11.

Indicators of the fourth $f_{4}^{\Delta}\left(X_{4}^{*}\right), f_{4}^{\Delta}\left(X_{4}^{o}\right)$, of characteristics of the system (are highlighted in red color) define transition errors from four-dimensional $X^{0}=\left\{x_{1}, x_{2}, x_{3}, x_{4}\right\}$ to two-dimensional $X^{o}=\left\{x_{1}, x_{2}\right\}$ to system of coordinates.

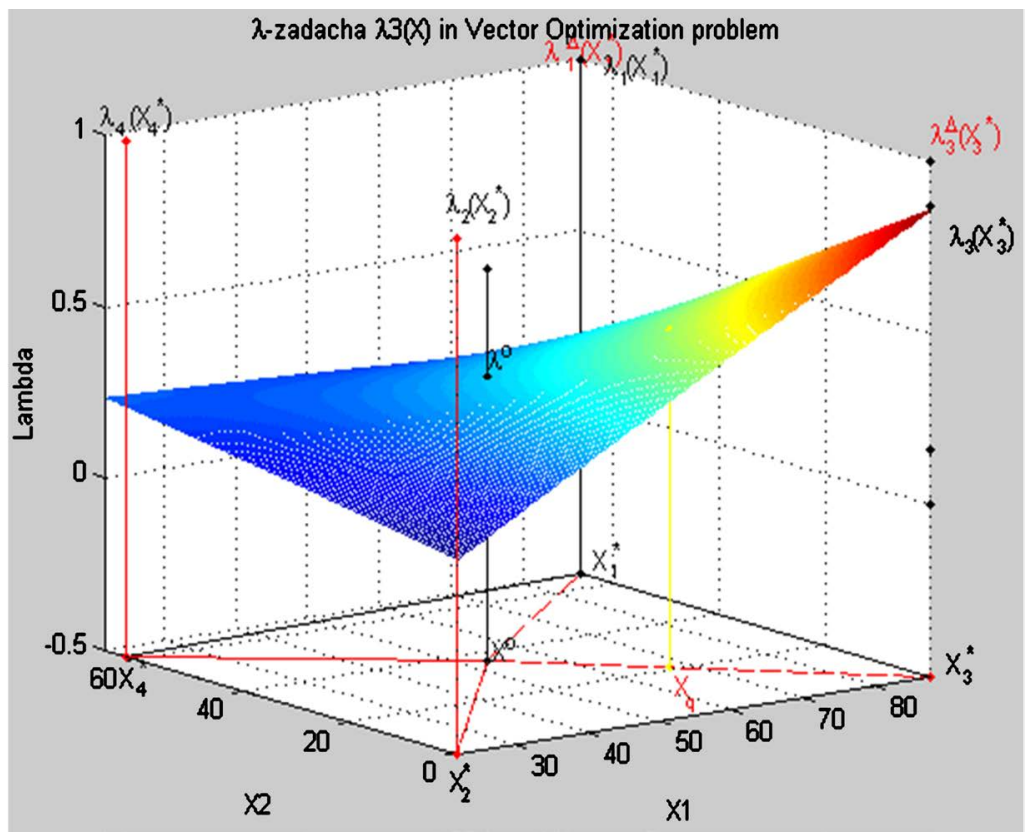

Figure 9. The third characteristics $\lambda_{3}(X)$ in coordinates $x_{1}, x_{2}$ technical system in the relative estimates.

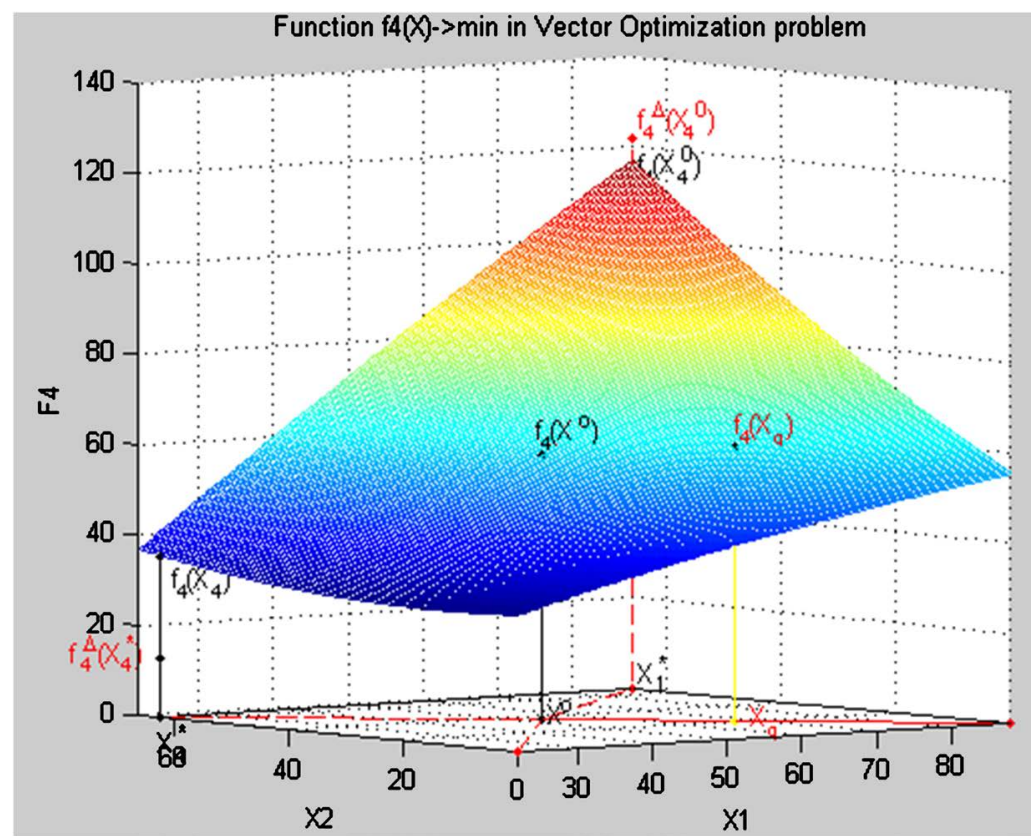

Figure 10. The fourth characteristics $f_{4}(X)$ in coordinates $x_{1}, x_{2}$ technical system in physical terms. 


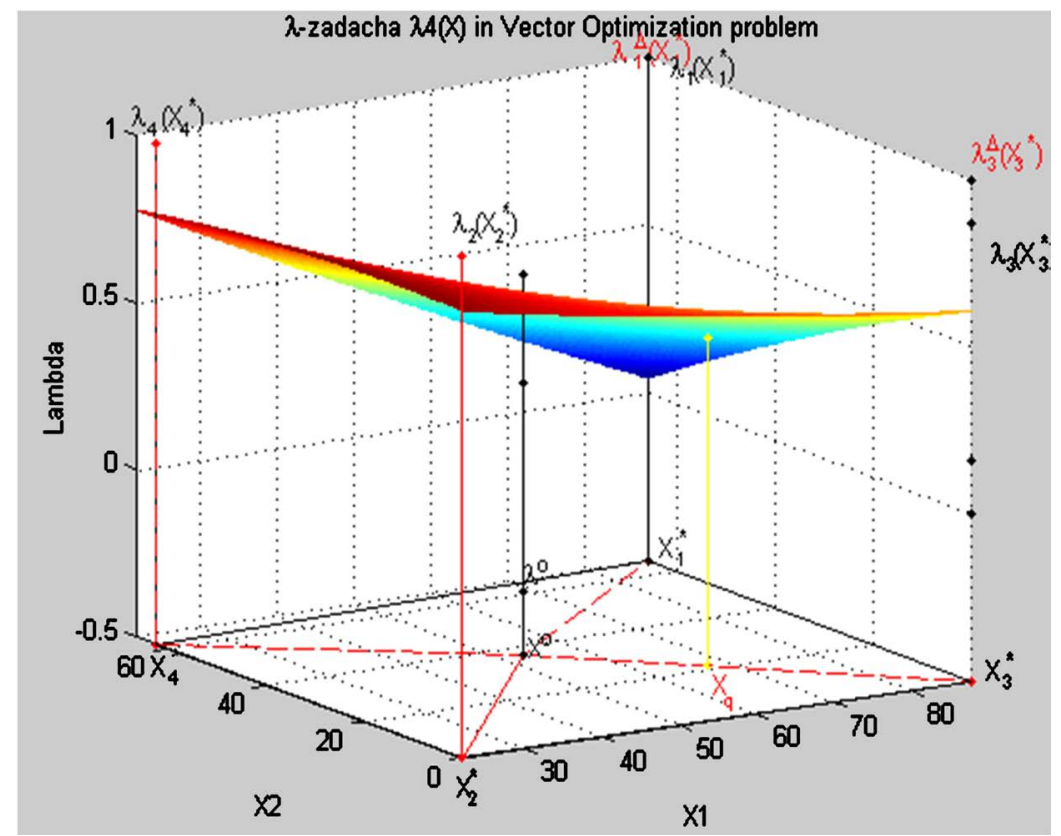

Figure 11. The fourth characteristics $\lambda_{4}(X)$ in coordinates $x_{1}, x_{2}$ technical system in the relative estimates.

Collectively, the submitted version:

- point $-X^{o}$; characteristics of $F\left(X^{o}\right)=\left\{f_{1}\left(X^{o}\right), f_{2}\left(X^{o}\right), f_{3}\left(X^{o}\right), f_{4}\left(X^{o}\right)\right\}$;

- relative estimates of $\lambda\left(X^{o}\right)=\left\{\lambda_{1}\left(X^{o}\right), \lambda_{2}\left(X^{o}\right), \lambda_{3}\left(X^{o}\right), \lambda_{4}\left(X^{o}\right)\right\}$;

- maximum $\lambda^{o}$ relative level such that $\lambda^{o} \leq \lambda_{k}\left(X^{o}\right) \quad \forall k \in \boldsymbol{K}$.

There is an optimum decision at equivalent criteria (characteristics), and procedure of receiving is adoption of the optimum decision at equivalent criteria (characteristics).

- point $-X^{q}$; characteristics of $F\left(X^{q}\right)=\left\{f_{1}\left(X^{q}\right), f_{2}\left(X^{q}\right), f_{3}\left(X^{q}\right), f_{4}\left(X^{q}\right)\right\}$;

- relative estimates of $\lambda\left(X^{o}\right)=\left\{\lambda_{1}\left(X^{q}\right), \lambda_{2}\left(X^{q}\right), \lambda_{3}\left(X^{q}\right), \lambda_{4}\left(X^{q}\right)\right\}$;

- maximum $\lambda^{o}$ relative level such that $\lambda^{o} \leq p_{k}^{q} \lambda_{k}\left(X^{q}\right), k=\overline{1, K}$.

There is an optimal solution at the set priority of the $q$-th criterion (characteristic) in relation to other criteria. Procedure of receiving a point is $X^{q}$ adoption of the optimal solution at the set priority of the second criterion.

Theory of vector optimization, methods of solution of the vector problems with equivalent criteria and given priority of criterion can choose any point from the set of points, optimum across Pareto, and show the optimality of this point.

\subsection{Methodology for Selecting of the Optimal Parameters of Technological Process under Conditions of Certainty and Uncertainty Based on Vector Optimization}

We study a technological process for which data are known about a certain set of functional characteristics (certainty conditions), discrete values of characteristics (uncertainty condition) and restrictions imposed on the functioning of 
the technological process. The numerical problem of modeling a technological process is solved with equivalent criteria and with a given priority of the criterion.

Stage 1. The technical assignment: "The choice of the optimal parameters of the technological process".

It is given. The technological process, the operation of which is determined by two parameters

$X=\left\{x_{1}, x_{2}\right\}$-the vector of variables (controlled). The operation of the process is determined by four characteristics (criteria)

$F(X)=\left\{f_{1}(X), f_{2}(X), f_{3}(X), f_{4}(X)\right\}$, the value of which depends on the vector of parameters $X$.

Conditions of certainty. The conditions are characterized by the fact that the functional dependence of the fourth characteristic $f_{4}(X)$ on the parameters of the technological process $X=\left\{x_{1}, x_{2}\right\}$ is known:

$$
\begin{aligned}
f_{4}(X)= & -0.2450-0.7470 x_{1}+0.3832 x_{1}^{2}+0.0442 x_{2} \\
& +0.0012 x_{2}^{2}-0.0346 x_{1} * x_{2}
\end{aligned}
$$

Conditions of uncertainty. For the first, second and third characteristics of the technological process, the results of experimental data are known: the values of the parameters and the corresponding characteristics. The numerical values of the parameters $X$ and the characteristics $y_{1}(X), y_{2}(X), y_{3}(X)$ are presented in Table 2.

In the decision taken, the evaluation value for the first, second and third characteristics (criteria) are desirable to get as high as possible:

$y_{1}(X) \rightarrow \max , y_{2}(X) \rightarrow \min , y_{3}(X) \rightarrow \max , f_{4}(X) \rightarrow \min$. The parameters $X=\left\{x_{1}, x_{2}\right\}$ vary within the following limits:

$$
2.0 \leq x_{1} \leq 3.5,12.0 \leq x_{2} \leq 30.0 .
$$

It is required. Build a model of the technological process in the form of a vector problem. Solve the vector problem with equivalent criteria. Choose a priority criterion. Set the numerical value of the priority criterion. Make the best (optimal) solution.

Note. The author has developed software for four parameters:

$X=\left\{x_{1}, x_{2}, x_{3}, x_{4}\right\}$ and six characteristics $F(X)=\left\{f_{1}(X), \cdots, f_{6}(X)\right\}$. For each task, the program is configured individually. If desired, the author can increase the number of parameters to five: $X=\left\{x_{1}, \cdots, x_{5}\right\}$. In the model, criteria with uncertainty conditions can vary from zero to six.

Stage 2. Construction of a mathematical model of the technological process [11] [25].

2.1. Construction in the conditions of a certainty. We form a vector problem for which the criteria (5) and restrictions (6) on the process parameters are known:

$$
\begin{aligned}
f_{4}(X)= & -0.2450-0.7470 x_{1}+0.3832 x_{1}^{2}+0.0442 x_{2}+0.0012 x_{2}^{2} \\
& -0.0346 x_{1} * x_{2}, \quad 2.0 \leq x_{1} \leq 3.5,12.0 \leq x_{2} \leq 30.0 .
\end{aligned}
$$


Table 2. Experimental weld input and output parameters.

\begin{tabular}{|c|c|c|c|c|}
\hline $\begin{array}{l}\text { Laser Power, } \mathrm{p} \\
\quad(\text { Analog V) }\end{array}$ & $\begin{array}{l}\text { Travel Speed, } \mathbf{v} \\
\quad(\mathrm{mm} / \mathrm{sec})\end{array}$ & $\begin{array}{l}\text { Wire Feed Rate, } \\
\qquad \mathrm{r}(\mathrm{m} / \mathrm{min})\end{array}$ & Depth, $D(m m)$ & $\begin{array}{l}\text { Total Accumulated Pore } \\
\text { Length, Po ( } \mathrm{mm} / \mathrm{mm})\end{array}$ \\
\hline$x_{1}$ & $x_{2}$ & $y_{1}(X) \rightarrow \max$ & $y_{2}(X) \rightarrow \min$ & $y_{3}(X) \rightarrow \max$ \\
\hline 2.40 & 24.2 & 4.2197 & 55.3951 & -0.0365 \\
\hline 2.76 & 18.72 & 3.2714 & 31.2497 & 0.0286 \\
\hline 2.76 & 19.08 & 3.2770 & 32.3886 & 0.0271 \\
\hline 2.76 & 31.68 & 4.2613 & 86.8526 & 0.0760 \\
\hline 2.76 & 31.92 & 4.2949 & 88.1656 & 0.0787 \\
\hline 3.30 & 14.40 & 3.0959 & 21.1331 & 0.3467 \\
\hline 3.30 & 25.20 & 3.0101 & 56.1913 & 0.2171 \\
\hline 3.30 & 25.80 & 3.0383 & 58.7506 & 0.2138 \\
\hline 3.30 & 26.76 & 3.0908 & 62.9794 & 0.2096 \\
\hline 3.30 & 27.60 & 3.1441 & 66.8147 & 0.2068 \\
\hline 3.30 & 28.80 & 3.2320 & 72.5126 & 0.2041 \\
\hline 3.30 & 30.00 & 3.3338 & 78.4682 & 0.2032 \\
\hline 3.30 & 31.20 & 3.4495 & 84.6812 & 0.2039 \\
\hline 3.30 & 32.40 & 3.5792 & 91.1518 & 0.2063 \\
\hline 3.30 & 36.00 & 4.0517 & 112.1086 & 0.2236 \\
\hline 3.84 & 18.72 & 3.0983 & 35.3082 & 0.6402 \\
\hline 3.84 & 23.52 & 2.9671 & 51.6458 & 0.5450 \\
\hline 3.84 & 31.68 & 3.2554 & 88.8758 & 0.4451 \\
\hline 3.84 & 32.88 & 3.3520 & 95.3551 & 0.4369 \\
\hline 4.20 & 25.20 & 3.2370 & 60.4633 & 0.7810 \\
\hline $\operatorname{Max} 2.40$ & 14.4 & 2.9671 & 21.1331 & -0.0365 \\
\hline $\operatorname{Max} .4 .20$ & 36.0 & 4.2949 & 112.1086 & 0.7810 \\
\hline
\end{tabular}

2.2. Construction in the conditions of an uncertainty consists in using qualitative and quantitative descriptions of a technological process obtained on the basis of the "input-output" principle in Table 2. Converting information (initial data $\left.y_{1}(X), y_{2}(X), y_{3}(X)\right)$ into a functional form $f_{4}(X)$ is carried out by using mathematical methods (the regression analysis). The initial data of Table 2 are formed as a matrix $I$ in the MATLAB system:

$$
I=|X, Y|=\left\{x_{i 1}, x_{i 2}, y_{i 1}, y_{i 2}, y_{i 3}, i=\overline{1, M}\right\} .
$$

For each experimental data set $y_{k}, k=\overline{1,3}$ the regression function is constructed using the least squares method $\min \sum_{i=1}^{M}\left(y_{i}-\overline{y_{i}}\right)^{2}$ in the MATLAB system. For this, the polynomial $A_{k}$ is formed, which determines the interrelation of the parameters $X_{i}=\left\{x_{i 1}, x_{i 2}\right\}$ and the function $\overline{y_{k i}}=f\left(X_{i}, A_{k}\right), k=\overline{1,3}$. 
The result is a system of coefficients $A_{k}=\left\{A_{0 k}, A_{1 k}, \cdots, A_{9 k}\right\}$, which determine the coefficients of the polynomial (function):

$$
f_{k}(X, A)=a_{0 k}+a_{1 k} x_{1}+a_{2 k} x_{1}^{2}+a_{3 k} x_{2}+a_{4 k} x_{2}^{2}+a_{5 k} x_{1} * x_{2}, k=\overline{1,3}
$$

The experimental data $I_{1}=\left\{x_{i 1}, x_{i 2}, y_{i 1}\right\}$ of the matrix $I=|X, Y|$ (84) are converted into the function $f_{1}(X)$ in the form (85), which, taking into account the obtained coefficients, takes the form:

$$
\begin{aligned}
f_{1}(X)= & 11.4745-4.8992 x_{1}+0.8868 x_{1}^{2}-0.0030 x_{2} \\
& +0.0048 x_{2}^{2}-0.0595 x_{1} * x_{2}
\end{aligned}
$$

The experimental data $I_{2}=\left\{x_{i 1}, x_{i 2}, y_{i 2}\right\}$ of the matrix $I=|X, Y|$ (84) are converted into the function $f_{2}(X)$ in the form (85), which, taking into account the obtained coefficients, takes the form:

$$
\begin{aligned}
f_{2}(X)= & 8.8176-7.6809 x_{1}+2.1456 x_{1}^{2}+0.1851 x_{2} \\
& +0.0894 x_{2}^{2}-0.1454 x_{1} * x_{2}
\end{aligned}
$$

The experimental data $I_{3}=\left\{x_{i 1}, x_{i 2}, y_{i 3}\right\}$ of the matrix $I=|X, Y|$ (84) are converted to the function $f_{3}(X)$ in the form (85), which, taking into account the obtained coefficients, takes the form:

$$
\begin{aligned}
f_{3}(X)= & -0.1225-0.3735 x_{1}+0.1916 x_{1}^{2}+0.0221 x_{2} \\
& +0.0006 x_{2}^{2}-0.0173 x_{1} * x_{12}
\end{aligned}
$$

2.3. Construction of a mathematical model of the technological process (General part for the conditions of certainty and uncertainty). To build a mathematical model of a technological process, we use: the functions obtained by the conditions of certainty and uncertainty (86), (87), (88); parametrical restrictions (82).

Functions (86), (87), (88) are considered as criteria determining the purposefulness of the operation of the technological process. All criteria are aimed $K_{1}=2$ at maximization: $f_{1}(X), f_{3}(X) \rightarrow \max$ and $K_{2}=2$ at minimization: $f_{2}(X), f_{4}(X) \rightarrow \max \quad \boldsymbol{K}=\boldsymbol{K}_{1} \cup \boldsymbol{K}_{2}$. As a result, the model of the functioning of the technological process will be represented by the vector problem of mathematical programming:

$$
\begin{aligned}
& \text { opt } F(X)=\left\{\max F_{1}(X)=\left\{\max f_{1}(X) \equiv 11.47-4.8992 x_{1}+0.8868 x_{1}^{2}\right.\right. \\
& -0.0030 x_{2}+0.0048 x_{2}^{2}-0.0595 x_{1} x_{2}, \\
& \max f_{3}(X) \equiv-0.1225-0.3735 x_{1}+0.1916 x_{1}^{2}+0.0221 x_{2} \\
& +0.0006 x_{2}^{2}-0.0173 x_{1} x_{2}, \\
& \min F_{2}(X)=\left\{\min f_{2}(X) \equiv 8.8176-7.6809 x_{1}+2.1456 x_{1}^{2}\right. \\
& +0.1851 x_{2}+0.0894 x_{2}^{2}-0.1454 x_{1} x_{2}, \\
& \min f_{4}(X) \equiv-0.2450-0.7470 x_{1}+0.3832 x_{1}^{2}+0.0442 x_{2} \\
& \left.\left.+0.0012 x_{2}^{2}-0.0346 x_{1} x_{2}\right\}\right\} \text {, }
\end{aligned}
$$


The vector problem of mathematical programming (89)-(93) represents the model of optimal decision making under certainty and uncertainty in the aggregate.

Stage 3. The solution of the vector problem of mathematical programmingthe model of the technological process.

To solve the vector problem of mathematical programming (89)-(93), we use the solution method based on the normalization of criteria and the principle of guaranteed results, presented in Section 3.3 [16]. The solution of the vector problem (89)-(93) with equivalent criteria is represented as a sequence of steps.

Step 1. Solving problem (89)-(93) for each criterion separately, using the fmincon(...) function of the MATLAB system [19], the call to the fmincon(...) function is considered in [15].

As a result of the calculation for each criterion, we obtain optimum points: $X_{k}^{*}$ and $f_{k}^{*}=f_{k}\left(X_{k}^{*}\right), k=\overline{1, K}, K=4$ is the values of the criteria at this point, that is, the best solution for each criterion:

$$
\begin{aligned}
& X_{1}^{*}=\left\{x_{1}=2.0, x_{2}=30.0\right\}, f_{1}^{*}=f_{1}\left(X_{1}^{*}\right)=-5.8833 ; \\
& X_{2}^{*}=\left\{x_{1}=3.5, x_{2}=30.0\right\}, f_{2}^{*}=f_{2}\left(X_{2}^{*}\right)=-78.9641 ; \\
& X_{3}^{*}=\left\{x_{1}=3.5, x_{2}=12.0\right\}, f_{3}^{*}=f_{3}\left(X_{3}^{*}\right)=-0.54235 ; \\
& X_{4}^{*}=\left\{x_{1}=2.9, x_{2}=12.0\right\}, f_{4}^{*}=f_{4}\left(X_{4}^{*}\right)=-0.3334 .
\end{aligned}
$$

The constraints (93) and optimum points $X_{1}^{*}, X_{2}^{*}, X_{3}^{*}, X_{4}^{*}$ (94) in the coordinates $\left\{x_{1}, x_{2}\right\}$ are shown in Figure 12. The set of valid points $S$ is not empty and is a compact:

$$
\boldsymbol{S}=\left\{X \in \boldsymbol{R}^{N} \mid 2.0 \leq x_{1} \leq 3.5,12.0 \leq x_{2} \leq 30.0\right\} \neq \varnothing
$$

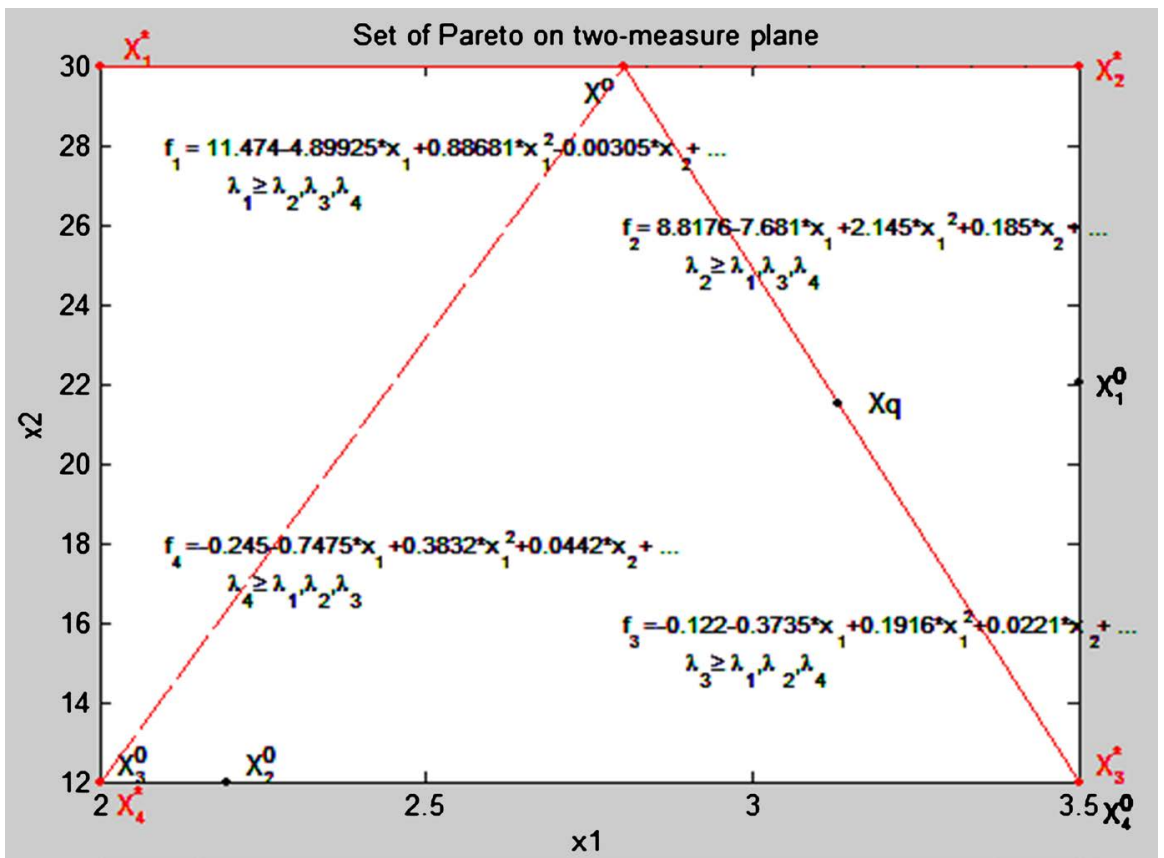

Figure 12. Pareto set, $S^{o} \subset S$ in two-dimensional coordinate system. 
The set of points that are Pareto optimal, $S^{o}$ represents the area of the set of points that lie between the points of the optimum $X_{1}^{*}, X_{2}^{*}, X_{3}^{*}, X_{4}^{*}$. We see that in this problem, the set of admissible points $S$ and the set of points optimal in Pareto, $S^{\boldsymbol{o}}$, are equal to each other: $S=\boldsymbol{S}^{\boldsymbol{0}}$.

Step 2. The worst unchanging part of each criterion is determined (anti-optimum) (a superscript zero):

$$
\begin{aligned}
& X_{1}^{0}=\left\{x_{1}=3.50, x_{2}=22.0052\right\}, f_{1}^{0}=f_{1}\left(X_{1}^{0}\right)=2.8663 ; \\
& X_{2}^{0}=\left\{x_{1}=2.1952, x_{2}=12.0\right\}, f_{2}^{0}=f_{2}\left(X_{2}^{0}\right)=13.56 ; \\
& X_{3}^{0}=\left\{x_{1}=2.0, x_{2}=12.0\right\}, f_{3}^{0}=f_{3}\left(X_{3}^{0}\right)=-0.1667 ; \\
& X_{4}^{0}=\left\{x_{1}=3.5, x_{2}=12.0\right\}, f_{4}^{0}=f_{4}\left(X_{4}^{0}\right)=-1.0847 .
\end{aligned}
$$

Step 3. A systematic analysis of the set of Pareto optimal points (i.e. analysis by each criterion) is performed. At the optimum points $X^{*}=\left\{X_{1}^{*}, X_{2}^{*}, X_{3}^{*}, X_{4}^{*}\right\}$, the values of the objective functions $F\left(X^{*}\right)=\left\|f_{q}\left(X_{k}^{*}\right)\right\|_{q=\overline{1, K}}^{k=\overline{1, K}}$ are determined, the vector $D=\left(d_{1} d_{2} d_{3} d_{4}\right)^{\mathrm{T}}$ deviations for each criterion on the admissible set $S: \quad d_{k}=f_{k}^{*}-f_{k}^{0}, k=\overline{1,4}$,

$$
F\left(X^{*}\right)=\left\|\begin{array}{llll}
-5.8833 & -79.3272 & -0.0619 & 0.1238 \\
-3.1731 & -78.9641 & -0.3038 & 0.6077 \\
-3.3468 & -17.2060 & -0.5423 & 1.0847 \\
-4.4505 & -13.6434 & 0.1667 & -0.3334
\end{array}\right\|, \quad d_{k}=\left\|\begin{array}{c}
3.0170 \\
65.4035 \\
0.7090 \\
1.4181
\end{array}\right\|
$$

and the relative estimation matrix $\lambda\left(X^{*}\right)=\left\|\lambda_{q}\left(X_{k}^{*}\right)\right\|_{q=\overline{1, K}}^{k=\overline{1, K}}$, where

$$
\begin{aligned}
& \lambda_{k}(X)=\left(f_{k}^{*}-f_{k}^{0}\right) / d_{k} \\
& \lambda\left(X^{*}\right)=\left\|\begin{array}{cccc}
1.0000 & 1.0056 & 0.3224 & 0.6776 \\
0.1017 & 1.0000 & 0.6636 & 0.3364 \\
0.1593 & 0.0557 & 1.0000 & 0 \\
0.5251 & 0.0013 & 0 & 1.0000
\end{array}\right\| .
\end{aligned}
$$

The analysis of the values of criteria (96) in relative estimates (97) shows that at the points of optimum $X^{*}=\left\{X_{1}^{*}, X_{2}^{*}, X_{3}^{*}, X_{4}^{*}\right\}$ (diagonally) the relative estimate is equal to one. The remaining criteria are significantly less than one. It is required to find a point (parameters) at which the relative estimates are closest to unity. The solution of this problem is aimed at solving the $\lambda$-problem-step 4 .

Step 4. The construction of the $\lambda$-problem is carried out in two stages: a maximin optimization problem with normalized criteria is initially constructed:

$$
\lambda^{o}=\max _{X \in S} \min _{k \in K} \lambda_{k}(X), G(X) \leq 0, X \geq 0 .
$$

At the second stage, the maximin problem (98) is transformed into a standard mathematical programming problem ( $\lambda$-problem):

$$
\lambda^{\circ}=\max \lambda,
$$

at restrictions 


$$
\begin{gathered}
\lambda-\frac{-11.47-4.8992 * x_{1}+0.8868 * x_{1}^{2}-0.0030 * x_{2}+0.0048 * x_{2}^{2}-0.0595 * x_{1} * x_{2}-f_{1}^{0}}{f_{1}^{*}-f_{1}^{0}} \leq 0, \\
\lambda-\frac{-0.1225-0.3735 * x_{1}+0.1916 * x_{1}^{2}+0.221 * x_{2}+0.0006 * x_{2}^{2}+0.0173 * x_{1} * x_{2}-f_{3}^{0}}{f_{3}^{*}-f_{3}^{0}} \leq 0, \\
\lambda-\frac{8.8176+7.6809 * x_{1}-2.1456 * x_{3}^{2}+0.1851 * x_{2}+0.0894 * x_{2}^{2}+0.1454 * x_{1} * x_{2}-f_{2}^{0}}{f_{2}^{*}-f_{2}^{0}} \leq 0, \\
\lambda-\frac{-0.2450-0.7470 * x_{1}-0.3832 * x_{1}^{2}+0.0442 * x_{2}+0.001245 * x_{2}^{2}+0.0345 * x_{1} * x_{2}-f_{4}^{0}}{f_{4}^{*}-f_{4}^{0}} \leq 0, \\
0 \leq \lambda \leq 1,2.0 \leq x_{1} \leq 3.5,12.0 \leq x_{2} \leq 30.0
\end{gathered}
$$

where the vector of unknowns has dimension of $N+1: \quad \boldsymbol{X}=\left\{x_{1}, \cdots, x_{N}, \lambda\right\}, N=$ 2 .

Step 5. Solution of the $\lambda$-problem. For this purpose we use the function fmincon(...) [19]:

[Xo] $[\mathrm{Lo}]=$ fmincon ('Z_TS_2Krit_L', X0, Ao, bo, Aeq, beq, lbo, ubo, 'Z_TS_LConst', options).

As a result of the solution of VPMP (89)-(93) at equivalent criteria and $\lambda$-problem corresponding to it (99)-(104) received:

$$
\boldsymbol{X}^{o}=\left\{X^{o}, \lambda^{o}\right\}=\left\{X^{o}=\left\{x_{1}=2.8039, x_{2}=30.0\right\}, \lambda^{o}=0.3541\right\}
$$

shown in Figure 12;

$f_{k}\left(X^{o}\right), k=\overline{1, K}_{\pi}$-values of criteria (characteristics of the technological process):

$$
\begin{aligned}
F\left(X^{o}\right)=\left\{f_{1}\left(X^{o}\right)\right. & =3.9345, f_{2}\left(X^{o}\right)=77.9319, \\
f_{3}\left(X^{o}\right) & \left.=0.0844, f_{4}\left(X^{o}\right)=0.1687\right\} ;
\end{aligned}
$$

$\lambda_{k}\left(X^{o}\right), k=\overline{1, K}_{\pi}$-values of relative estimates

$$
\begin{aligned}
\lambda\left(X^{o}\right)=\left\{\lambda_{1}\left(X^{o}\right)\right. & =0.3541, \lambda_{2}\left(X^{o}\right)=0.9842, \\
\lambda_{3}\left(X^{o}\right) & \left.=0.3541, \lambda_{4}\left(X^{o}\right)=0.6459\right\} ;
\end{aligned}
$$

$\lambda^{\circ}=0.3541$ is the maximum lower level among all relative estimates, measured in the relative units:

$\lambda^{o}=\min \left\{\lambda_{1}\left(X^{o}\right), \lambda_{2}\left(X^{o}\right), \lambda_{3}\left(X^{o}\right), \lambda_{4}\left(X^{o}\right)\right\}=0.3541, \lambda^{o}$-also called a guaranteed result in the relative units, i.e., $\lambda_{k}\left(X^{0}\right)$ and, accordingly, the characteristics of the technological process $f_{k}\left(X^{\circ}\right)$ it is impossible to improve, without worsening at the same time other characteristics. Note that, in accordance with Theorem 1 , at point $\boldsymbol{X}^{\boldsymbol{0}}$ (105), criteria 1 and 3 are contradictory. This contradiction is determined by the equality $\lambda_{1}\left(X^{o}\right), \lambda_{3}\left(X^{o}\right)=\lambda^{\circ}=0.3541$ (107), and the remaining criteria by the inequality $\left\{\lambda_{2}\left(X^{o}\right)=0.9842, \lambda_{4}\left(X^{o}\right)=0.6459\right\}>\lambda^{o}$.

Thus, Theorem 1 serves as the basis for determining the correctness of the solution of the vector problem.

In the vector problem of mathematical programming, as a rule, for two criteria, equality is satisfied: 


$$
\lambda^{o}=\lambda_{q}\left(X^{o}\right)=\lambda_{p}\left(X^{o}\right), q, p \in \boldsymbol{K}, X \in S,
$$

and for other criteria it is defined as inequality: $\left\{\lambda_{2}\left(X^{\circ}\right), \lambda_{4}\left(X^{\circ}\right)\right\}>\lambda^{\circ}$.

Stage 4. Creation of geometrical interpretation of results of the decision in a three-dimensional coordinate. In the allowable set of points $S$ formed by constraints (93), the optimum points $X^{*}=\left\{X_{1}^{*}, X_{2}^{*}, X_{3}^{*}, X_{4}^{*}\right\}$, which are shown in Figure 12, combined into a contour, represent the set of Pareto optimal points, $\boldsymbol{S}^{o} \subset \boldsymbol{S}$. The coordinates of these points, as well as the characteristics of the technological process in relative units $\lambda_{1}(X), \lambda_{2}(X), \lambda_{3}(X), \lambda_{4}(X)$ are shown in Figure 13 in the three-dimensional space $x_{1}, x_{2}$ and $\lambda$, where the third axis is the relative estimate $\lambda$.

Stage 5. Decision-making in the technological process model at the set priority of criterion.

To solve vector problems of mathematical programming (21)-(26) methods are presented, based on axiomatic normalization of criteria and principle of guaranteed result, as well as axiomatic priority of criterion, resulting from axiom 2, 3 and principle of optimality 2, which are presented in Section 3.2 [16].

The decision maker is usually the process designer.

Step 1. We solve a vector problem (89)-(93) with equivalent criteria. The algorithm of the decision is presented in the stage 3. Numerical results of the solution of the vector problem are given above. Pareto's great number of $S^{o} \subset S$ lies between optimum points $X_{1}^{*}, X_{2}^{*}, X_{3}^{*}, X_{4}^{*}, X_{1}^{*}$. This information is the basis for further research on the structure of the Pareto set. The decision maker is usually the technological process designer. If results of the solution of a vector problem with equivalent criteria do not satisfy the person making the decision,

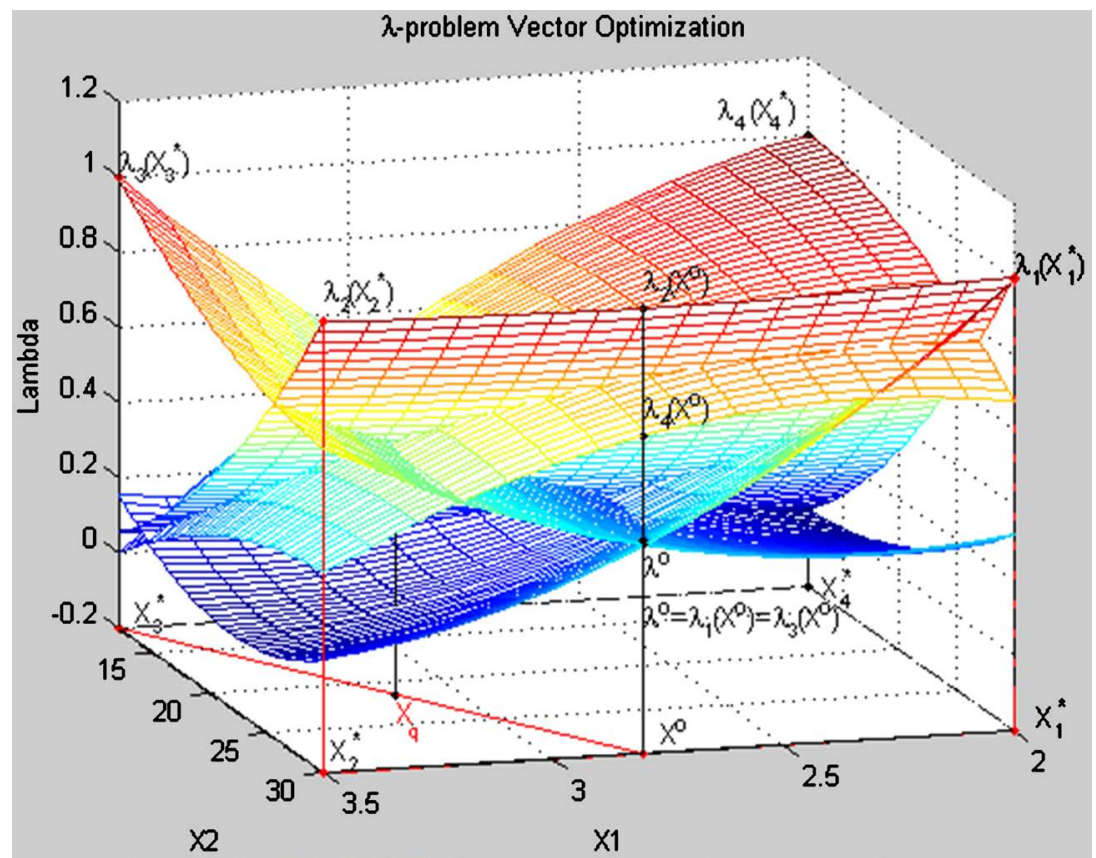

Figure 13. Geometric interpretation of the solution of the $\lambda$-problem coordinate $x_{1}, x_{2}$ and $\lambda$. 
then the choice of an optimal solution is carried out from any subset of points of $\boldsymbol{S}_{1}^{o}, \boldsymbol{S}_{2}^{o}, \boldsymbol{S}_{3}^{o}, \boldsymbol{S}_{4}^{o}$.

Step 2. Choice of priority criterion of $q \in \boldsymbol{K}$. From the theory (the Theorem 2 ) it is known that in an optimum point of $X^{0}$ there are always two most contradictory criteria: $q \in \boldsymbol{K}$ and $v \in \boldsymbol{K}$ for which in the relative unit's precise equality is carried out:

$$
\lambda^{o}=\lambda_{q}\left(X^{o}\right)=\lambda_{p}\left(X^{o}\right), q, p \in K, X \in S,
$$

and for the others it is carried out inequalities:

$$
\lambda^{o} \leq \lambda_{k}\left(X^{o}\right) \quad \forall k \in K \quad q \neq v \neq k .
$$

For the choice of the priority criterion on the display the message about results of the solution of $\lambda$-problem in physical and relative units is given:

Criteria (106) in $X^{o}$ optimum point:

$$
\begin{aligned}
F\left(X^{o}\right)=\left\{f_{1}\left(X^{o}\right)\right. & =3.9345, f_{2}\left(X^{o}\right)=77.9319, \\
f_{3}\left(X^{o}\right) & \left.=0.0844, f_{4}\left(X^{o}\right)=0.1687\right\} ;
\end{aligned}
$$

The relative estimates (107) in $X^{0}$ :

$$
\begin{aligned}
& \lambda\left(X^{o}\right)=\left\{\lambda_{1}\left(X^{o}\right)=0.3541, \lambda_{2}\left(X^{o}\right)=0.9842,\right. \\
& \left.\lambda_{3}\left(X^{\circ}\right)=0.3541, \lambda_{4}\left(X^{\circ}\right)=0.6459\right\} .
\end{aligned}
$$

From the function $\lambda\left(X^{o}\right)$ it is clear that the first and the third are the most contradictory criteria:

$$
\lambda^{o}=\left\{\lambda_{1}\left(X^{o}\right), \lambda_{3}\left(X^{o}\right)\right\}=0.3541,
$$

Select from Figure 2 the first and third criteria and present (30) $\left\{\lambda_{1}\left(X^{\circ}\right), \lambda_{3}\left(X^{o}\right)\right\}$ in the relative units in Figure 14 .

As a rule, from a pair of the contradictory criteria, a criterion chosen by the decision maker would be improved. Such criterion is called "priority criterion", we will designate it $q=3 \in \boldsymbol{K}$. This criterion is investigated in interaction with the first criterion of $k=1 \in \boldsymbol{K}$.

On the display the message is given:

$q=$ input ('Enter priority criterion (number) of $q=$ ') -Entered: $q=3$.

Step 3. Numerical limits of the change of the size of a priority of the criterion of $q=3 \in \boldsymbol{K}$ are defined.

For priority criterion of $q=3 \in \boldsymbol{K}$ changes of the numerical limits in the physical units upon transition from $X^{o}$ optimum point to the point of $X_{q}^{*}$ received on the first step at equivalent criteria are defined. $q=3$ given about criterion are given for the screen:

$$
f_{q}\left(X^{o}\right)=-0.084354 \leq f_{q}(X) \leq-0.54235=f_{q}\left(X_{q}^{*}\right), q=3 \in \boldsymbol{K} .
$$

In the relative units the criterion of $q=3$ changes in the following limits:

$$
\lambda_{q}\left(X^{o}\right)=0.35407 \leq \lambda_{q}(X) \leq 1=\lambda_{q}\left(X_{q}^{*}\right), q=3 \in \boldsymbol{K} .
$$




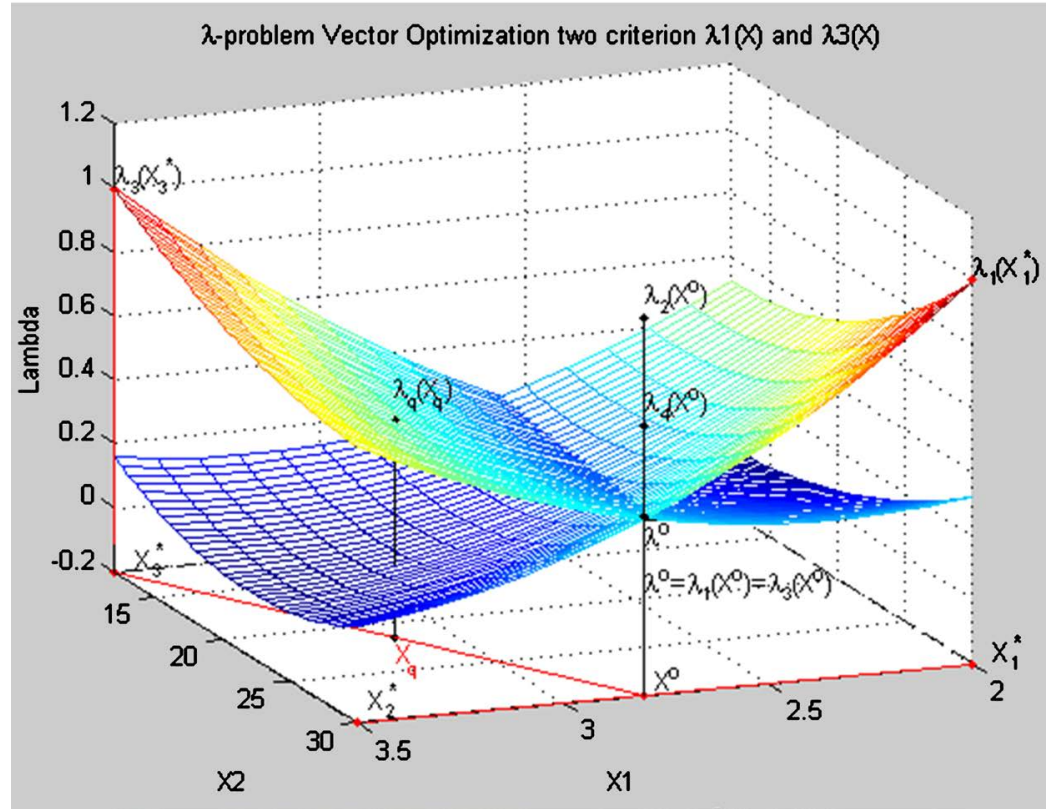

Figure 14. Result the solution of the problem (89)-(93) in the three-dimensional coordinate system $\left\{x_{1}, X_{2}, \lambda\right\}: \lambda_{1}(X), \lambda_{3}(X)$.

This data is analyzed.

Step 4. Choice of size of the priority criterion of $q \in \boldsymbol{K}$. (Decision-making). On the message: "Enter the size of priority criterion $f_{q}=$ "-we enter, the size of the characteristic $q=3 \in \boldsymbol{K}: f_{q}=0.3$.

Step 5. The relative assessment is calculated.

For the chosen size of priority criterion $f_{q}=0.3$ the relative assessment is calculated:

$$
\lambda_{q}=\frac{f_{q}-f_{q}^{0}}{f_{q}^{*}-f_{q}^{0}}=\frac{0.3-(-0.1667)}{0.54235-(-0.1667)}=0.6582,
$$

which upon transition from $X^{0}$ point to $X_{3}^{*}$ lies in the limits:

$$
0.35407=\lambda_{3}\left(X^{\circ}\right) \leq \lambda_{3}=0.6582 \leq \lambda_{3}\left(X_{3}^{*}\right)=1, q \in \boldsymbol{K}
$$

Step 6. Let's calculate coefficient of the linear approximation

Assuming the linear nature of the change of the criterion of $f_{q}(X)$ in (109) and according to the relative assessment of $\lambda_{q}$, using standard linear approximation techniques, we will calculate the proportionality coefficient between $\lambda_{q}\left(X^{0}\right), \lambda_{q}$ which we will call $\rho$ :

$$
\rho=\frac{\lambda_{q}-\lambda_{q}\left(X^{\circ}\right)}{\lambda_{q}\left(X_{q}^{*}\right)-\lambda_{q}\left(X^{\circ}\right)}=\frac{0.6582-0.35407}{1-0.35407}=0.4708, q=3 \in \boldsymbol{K} .
$$

Step 7. Let's calculate coordinates of a priority of the criteria with dimension of $f_{q}$

Assuming the linear nature of change of a vector of $X^{q}=\left\{x_{1} x_{3}\right\}, q=3$ we will determine point coordinates with dimension of $f_{q}=0.3$, the relative as- 
sessment $\lambda_{q}$ :

$x_{\lambda=0.7}^{q=2}=\left\{x_{1}=X^{o}(1)+\rho\left(X_{q}^{*}(1)-X^{o}(1)\right), x_{2}=X^{o}(2)+\rho\left(X_{q}^{*}(2)-X^{o}(2)\right)\right\}$,

where $X^{o}=\left\{X^{o}(1)=2.8039, X^{o}(2)=30.0\right\}$,

$X_{3}^{*}=\left\{X_{3}^{*}(1)=80.0, X_{3}^{*}(2)=0.0\right\}$.

As result of the decision (110) we will receive $X^{q}$ point with coordinates:

$$
X^{q}=\left\{x_{1}=3.1317, x_{2}=21.5248\right\} .
$$

Step 8. Calculation of the main indexes of a point of $X_{q}$. For the received $X^{q}$ point, we will calculate:

All criteria in the physical units $f_{k}\left(X^{q}\right)=\left\{f_{k}\left(X^{q}\right), k=\overline{1, K}\right\}$,

$$
\begin{aligned}
f\left(X^{q}\right)=\left\{f_{1}\left(X^{q}\right)=2.9775, f_{2}\left(X^{q}\right)=41.4096,\right. \\
\left.f_{3}\left(X^{q}\right)=0.1744, f_{4}\left(X^{q}\right)=0.3489\right\} ;
\end{aligned}
$$

All relative estimates with the criterion priority

$$
\begin{gathered}
\lambda^{q}=\left\{\lambda_{k}^{q}, k=\overline{1, K}\right\}, \lambda_{k}\left(X^{q}\right)=\frac{f_{k}\left(X^{q}\right)-f_{k}^{0}}{f_{k}^{*}-f_{k}^{0}}, k=\overline{1, K}, \\
\lambda_{k}\left(X^{q}\right)=\left\{\lambda_{1}\left(X^{q}\right)=0.0369, \lambda_{2}\left(X^{q}\right)=0.4258\right. \\
\left.\lambda_{3}\left(X^{q}\right)=0.4811, \lambda_{4}\left(X^{q}\right)=0.5189\right\} ;
\end{gathered}
$$

Minimum relative assessment: $\min \left(\lambda_{k}\left(X^{q}\right)\right)=0.0369$;

Vector of priorities of the third criterion over other criteria:

$$
\begin{aligned}
P^{q}(X)=\left\{p_{k}^{q}=\frac{\lambda_{q}\left(X^{q}\right)}{\lambda_{k}\left(X^{q}\right)}, k=\overline{1, K}\right\}, \\
P^{q}=\left[p_{1}^{3}=6.2789, p_{2}^{3}=17.94, p_{3}^{3}=1.0, p_{4}^{3}=0.9\right] ;
\end{aligned}
$$

Any point from Pareto's set $\boldsymbol{X}_{t}^{o}=\left\{\lambda_{t}^{o}, X_{t}^{o}\right\} \in \boldsymbol{S}^{o}$ can be similarly calculated.

Analysis of results. The calculated size of criterion $f_{q}\left(X_{t}^{o}\right), q \in \boldsymbol{K}$ is usually not equal to the set $f_{q}$. The error of the choice of $\Delta f_{q}=\left|f_{q}\left(X_{t}^{o}\right)-f_{q}\right|=|0.1744-0.3|=0.125$ is defined by an error of linear approximation, $\Delta f_{q \%}=\frac{\Delta f_{q}}{f_{q}} * 100=40.2 \%$.

In the course of the modeling parametrical restrictions (93) can be changed, i.e. some set of optimum decisions is received. Choose a final version which in our example included from this set of optimum decisions:

- parameters of technological process $X^{\circ}=\left\{x_{1}=2.8039, x_{2}=30.0\right\}$;

- the parameters of the technological system at a given priority criterion $q=3$ :

$$
X^{q}=\left\{x_{1}=3.1317, x_{2}=21.5248\right\} .
$$

Stage 6. Geometrical interpretation of results of the decision in three to a measured frame in physical units. 
We represent these parameters in a two-dimensional $x_{1}, x_{2}$ and three dimensional coordinate system $X_{1}, X_{2}$ and $\lambda$ in Figures 12-14, and also in physical units for each function $f_{1}(X), f_{2}(X), f_{3}(X), f_{4}(X)$ on Figures 15-18 respectively.

The first characteristic $f_{1}(X)$ in physical units show in Figure 15.

The second characteristic $f_{2}(X)$ in physical units show in Figure 16 .

The third characteristic $f_{3}(X)$ in physical units show in Figure 17.

The four characteristic $f_{4}(X)$ in physical units show in Figure 18.

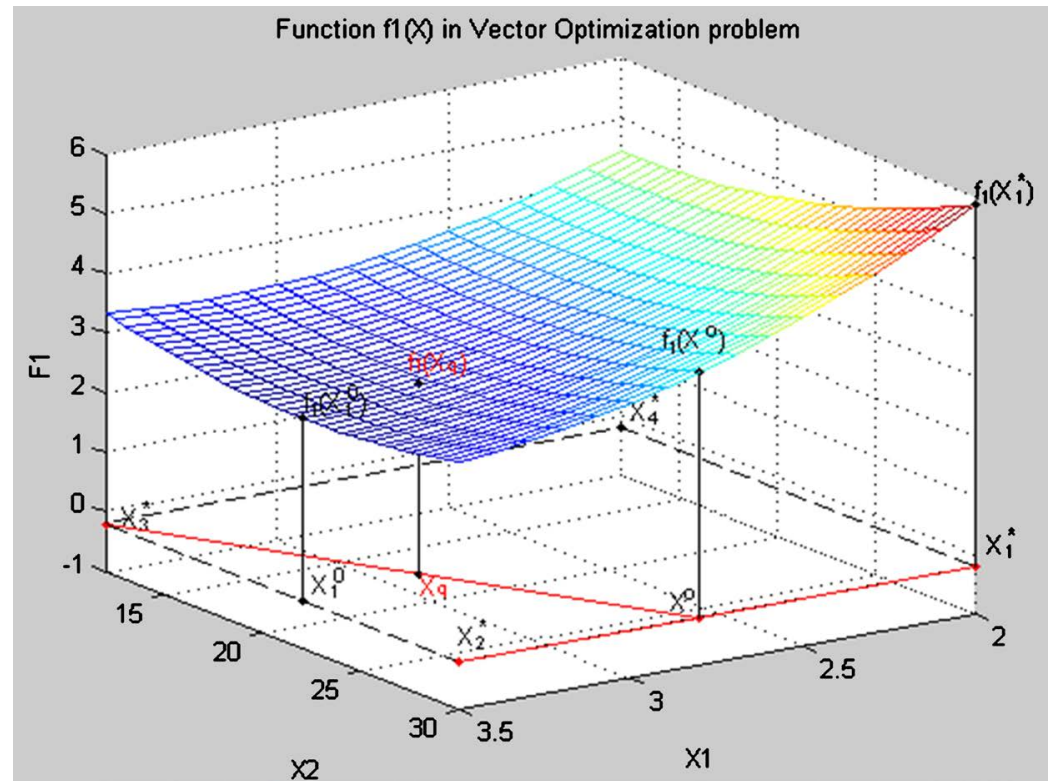

Figure 15. Result the solution of the problem (89)-(93) in the three-dimensional coordinate system $\left\{x_{1}, x_{2}\right.$ and $\left.f_{1}(X)\right\}$.

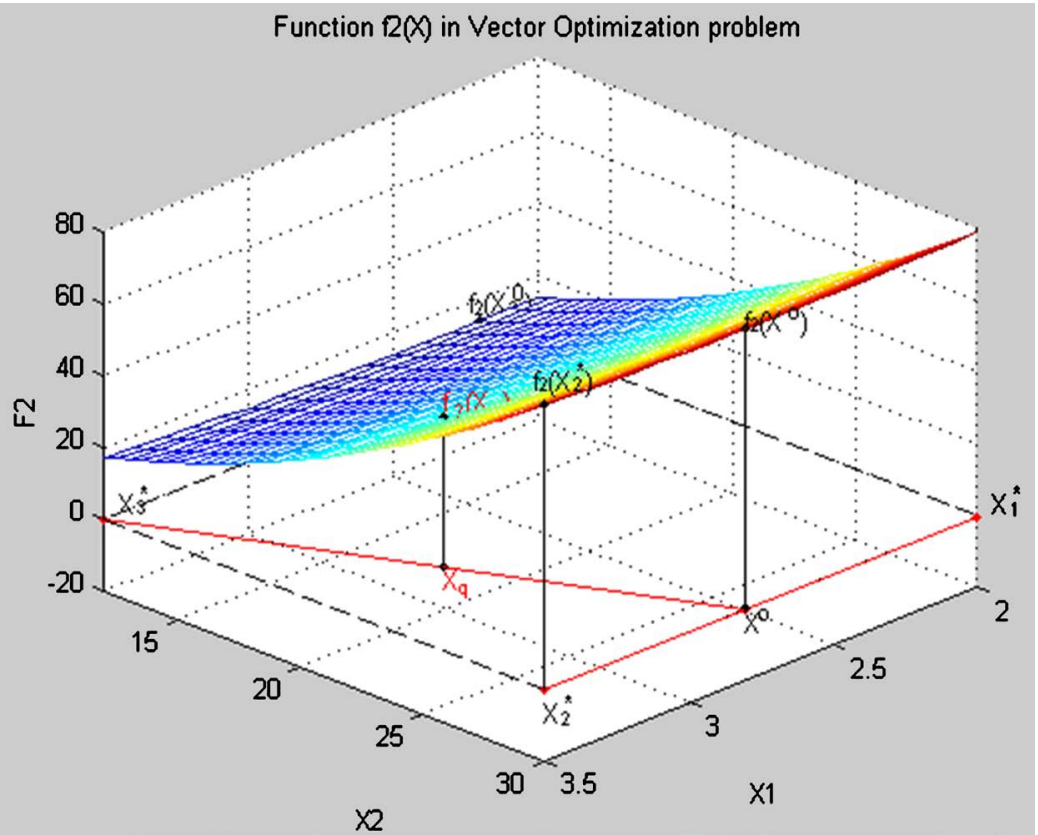

Figure 16. Result the solution of the problem (89)-(93) in the three-dimensional coordinate system $\left\{X_{1}, X_{2}\right.$ and $\left.f_{2}(X)\right\}$. 


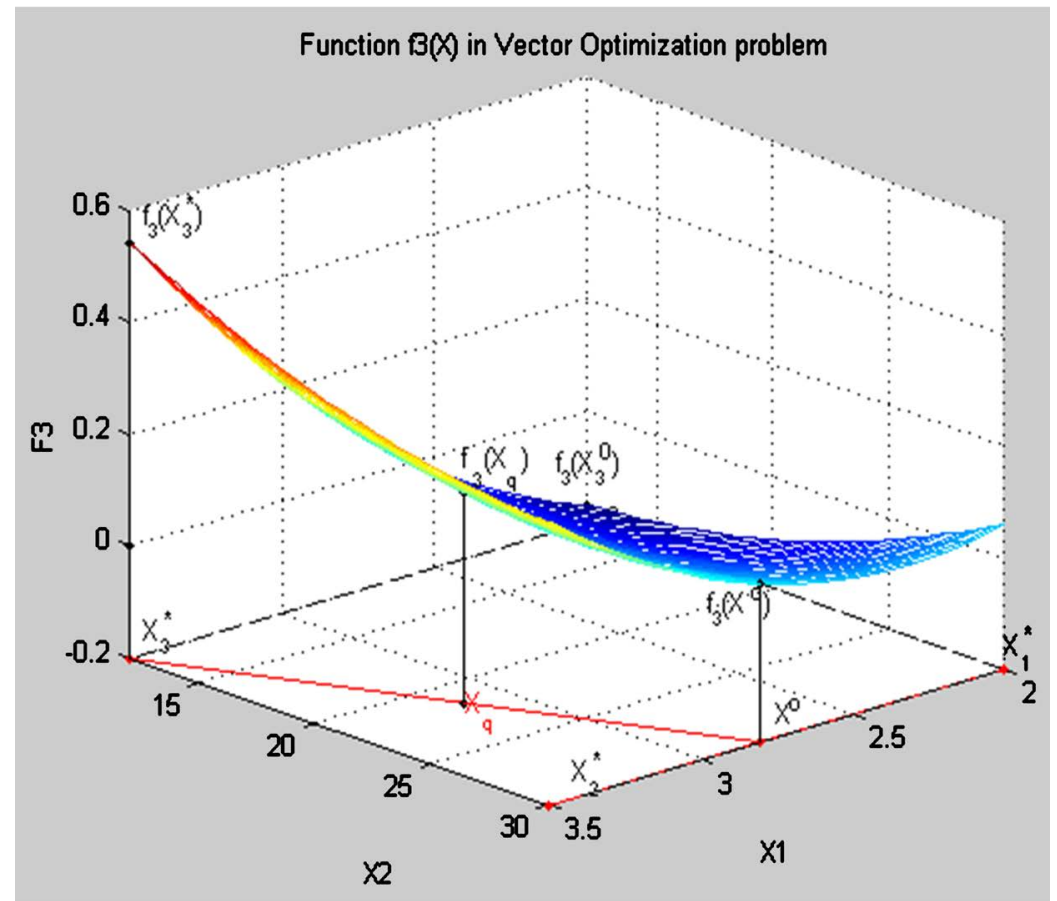

Figure 17. Result the solution of the problem (89)-(93) in the three-dimensional coordinate system $\left\{X_{1}, x_{3}\right.$ and $\left.f_{3}(X)\right\}$.

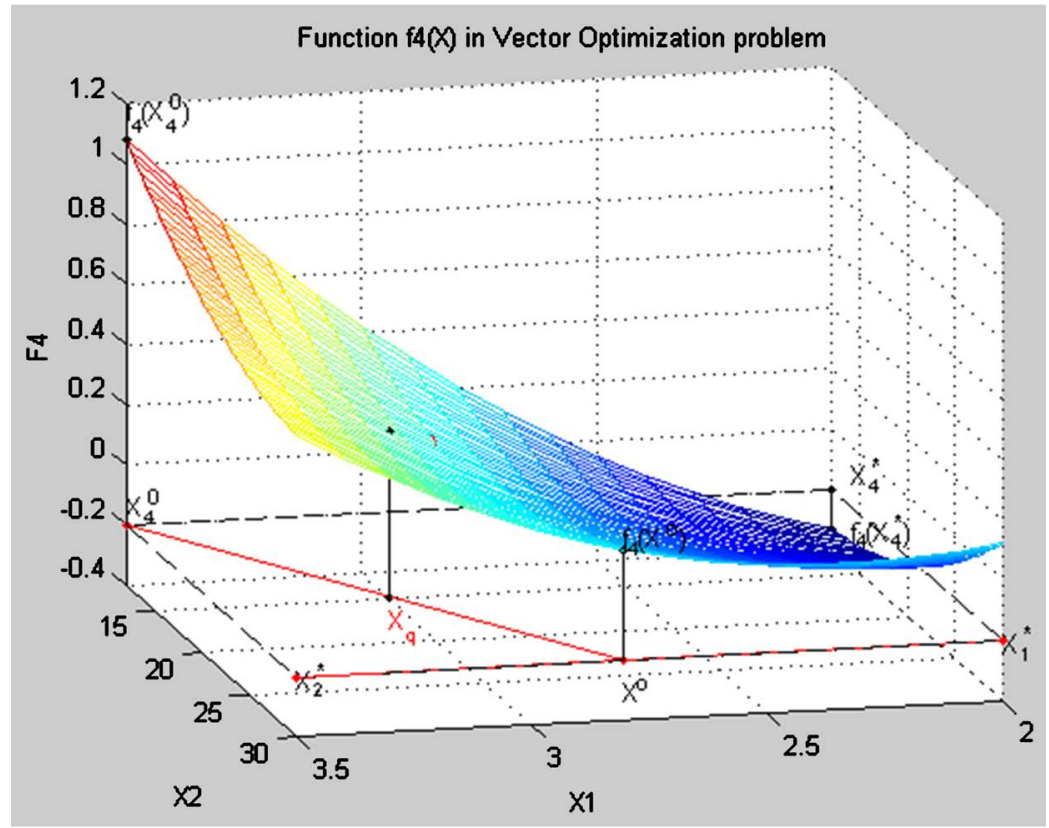

Figure 18. Result the solution of the problem (89)-(93) in the three-dimensional coordinate system $\left\{x_{1}, x_{2}\right.$ and $\left.f_{3}(X)\right\}$.

In the aggregate, the first option presented:

- point $X^{o}=\left\{x_{1}, x_{2}\right\}$;

- the functional characteristics $F\left(X^{o}\right)=\left\{f_{1}\left(X^{o}\right), f_{2}\left(X^{o}\right), f_{3}\left(X^{o}\right), f_{4}\left(X^{o}\right)\right\}$;

- relative estimates of $\lambda\left(X^{o}\right)=\left\{\lambda_{1}\left(X^{o}\right), \lambda_{2}\left(X^{o}\right), \lambda_{3}\left(X^{o}\right), \lambda_{4}\left(X^{o}\right)\right\}$; 
- maximin $\lambda^{o}$-relative level such that $\lambda^{o} \leq \lambda_{k}\left(X^{o}\right) \quad \forall k \in \boldsymbol{K}$.

There is an optimal solution with equivalent criteria (characteristics), and the procedure for obtaining is the adoption of the optimal solution in the process with equivalent criteria (characteristics).

The second option:

- point $-X^{q}=\left\{X^{1 q}, X^{2 q}\right\}$; characteristics

$$
f\left(X^{q}\right)=\left\{f_{1}\left(X^{q}\right), f_{2}\left(X^{q}\right), f_{3}\left(X^{q}\right), f_{4}\left(X^{q}\right)\right\} ;
$$

- relative estimates $\lambda_{k}\left(X^{q}\right)=\left\{\lambda_{1}\left(X^{q}\right), \lambda_{2}\left(X^{q}\right), \lambda_{3}\left(X^{q}\right), \lambda_{4}\left(X^{q}\right)\right\}$;

- maximin $\lambda^{o q}$ is a relative level such that

$$
\lambda^{o q} \leq \lambda_{1}\left(X^{q}\right), \lambda_{2}\left(X^{q}\right), \lambda_{3}\left(X^{q}\right), \lambda_{4}\left(X^{q}\right) ;
$$

There is an optimal solution with the priority of the third criterion (characteristics) relative to other criteria. The procedure for obtaining the point $X^{q}$ is the adoption of the optimal solution for a given priority of the criterion.

The vector optimization theory, methods for solving vector problems with equivalent criteria and a given criterion priority allow you to select any point from the set of Pareto optimal points and show the optimality of this point.

\subsection{Methodology for Selecting of the Optimal Parameters Structure of Material under Conditions of Certainty and Uncertainty Based on Vector Optimization}

The problem of a decision making of structure of material about which are known is considered: first, given about the functional interrelation of several characteristics with its components which are a part of this material ((the conditions of definiteness are absent); secondly, data on some set of discrete values of several characteristics (the experimental results), in interrelation with discrete values of the components which are a part of this material (uncertainty conditions).

Stage 1. The technical assignment: "The choice of optimum parameters of material".

Requirement specification: "The choice of optimum parameters of material" is carried out by the designer of material.

It is given. Material which structure is defined by four components: $Y=\left\{y_{1}, y_{2}, y_{3}, y_{4}\right\}$ - a vector (operated) variables. Input data for a decision making are four characteristics: $H(Y)=\left\{h_{1}(Y), h_{2}(Y), h_{3}(Y), h_{4}(Y)\right\}$. Conditions of a certainty are absent.

Conditions of uncertainty. For four characteristics are known discrete values of components (experimental) $Y=\left\{y_{1}, y_{2}, y_{3}, y_{4}\right\}$-with the corresponding discrete values of characteristics of $H(Y)=\left\{h_{1}(Y), h_{2}(Y), h_{3}(Y), h_{4}(Y)\right\}$. Numerical values of parameters $Y$ and characteristics of $H(X)$ are presented in Table 3.

Parametrical restrictions change in the following limits (as a percentage): $21 \leq y_{1} \leq 79, \quad 5 \leq y_{2} \leq 59,2.1 \leq y_{3} \leq 9,2.2 \leq y_{4} \leq 7$. On the parameters $Y=\left\{y_{1}, y_{2}, y_{3}, y_{4}\right\}$ are in total imposed restriction:

$$
y_{1}+y_{2}+y_{3}+y_{4}=100 \text {. }
$$


Table 3. Numerical values of components and characteristics of material.

\begin{tabular}{|c|c|c|c|c|c|c|c|}
\hline$y_{1}$ & $y_{2}$ & $y_{3}$ & $y_{4}$ & $h_{1}(Y)$ & $h_{2}(Y)$ & $h_{3}(Y)$ & $h_{4}(Y)$ \\
\hline 20 & 0 & 2 & 2 & 396.0 & 1149.6 & 100.08 & 24.24 \\
\hline 20 & 0 & 2 & 5 & 408.0 & 1164.0 & 99.600 & 27.60 \\
\hline 20 & 0 & 2 & 8 & 417.6 & 1176.0 & 99.480 & 28.80 \\
\hline 20 & 0 & 5 & 2 & 423.6 & 1212.0 & 103.32 & 30.00 \\
\hline 20 & 0 & 5 & 5 & 432.0 & 1260.0 & 99.000 & 31.20 \\
\hline 20 & 0 & 5 & 8 & 438.0 & 1257.6 & 98.520 & 32.40 \\
\hline 20 & 0 & 8 & 2 & 451.2 & 1256.4 & 96.240 & 33.60 \\
\hline 20 & 0 & 8 & 5 & 457.2 & 1252.8 & 94.920 & 34.80 \\
\hline 20 & 0 & 8 & 8 & 468.0 & 1251.6 & 94.320 & 34.80 \\
\hline 20 & 30 & 2 & 2 & 300.0 & 2143.2 & 111.60 & 19.92 \\
\hline 20 & 30 & 2 & 5 & 309.6 & 2154.0 & 110.76 & 21.60 \\
\hline 20 & 30 & 2 & 8 & 315.6 & 2163.6 & 110.28 & 25.20 \\
\hline 20 & 30 & 5 & 2 & 324.0 & 2176.8 & 109.68 & 29.76 \\
\hline 20 & 30 & 5 & 5 & 334.8 & 2185.2 & 108.12 & 33.48 \\
\hline 20 & 30 & 5 & 8 & 339.6 & 2198.4 & 107.88 & 37.20 \\
\hline 20 & 30 & 8 & 2 & 348.0 & 2211.6 & 107.76 & 39.48 \\
\hline 20 & 30 & 8 & 5 & 361.2 & 2232.0 & 105.60 & 42.00 \\
\hline 20 & 30 & 8 & 8 & 372.0 & 2245.2 & 105.84 & 49.20 \\
\hline 20 & 60 & 2 & 2 & 204.0 & 2954.4 & 130.80 & 15.60 \\
\hline 20 & 60 & 2 & 5 & 210.0 & 2820.0 & 126.00 & 18.00 \\
\hline 20 & 60 & 2 & 8 & 222.0 & 2772.0 & 122.40 & 21.60 \\
\hline 20 & 60 & 5 & 2 & 228.0 & 2748.0 & 120.48 & 24.24 \\
\hline 20 & 60 & 5 & 5 & 237.6 & 2832.0 & 122.40 & 28.80 \\
\hline 20 & 60 & 5 & 8 & 244.8 & 2904.0 & 124.80 & 32.40 \\
\hline 20 & 60 & 8 & 2 & 252.0 & 3022.8 & 126.96 & 35.16 \\
\hline 20 & 60 & 8 & 5 & 265.2 & 3036.0 & 126.00 & 39.60 \\
\hline 20 & 60 & 8 & 8 & 276.0 & 3056.4 & 125.04 & 44.88 \\
\hline 50 & 0 & 2 & 2 & 12.00 & 3583.2 & 157.68 & 11.28 \\
\hline 50 & 0 & 2 & 5 & 19.20 & 3601.2 & 157.20 & 14.40 \\
\hline 50 & 0 & 2 & 8 & 28.80 & 3608.4 & 156.00 & 16.80 \\
\hline 50 & 0 & 5 & 2 & 36.00 & 3616.8 & 155.76 & 21.12 \\
\hline 50 & 0 & 5 & 5 & 45.60 & 3622.8 & 154.80 & 22.80 \\
\hline 50 & 0 & 5 & 8 & 52.80 & 3637.2 & 154.44 & 27.60 \\
\hline 50 & 0 & 8 & 2 & 60.00 & 3651.6 & 153.84 & 30.84 \\
\hline 50 & 0 & 8 & 5 & 72.00 & 3672.0 & 152.40 & 36.00 \\
\hline 50 & 0 & 8 & 8 & 84.00 & 36852 & 151.92 & 40.56 \\
\hline 50 & 30 & 2 & 2 & 492.0 & 1195.2 & 107.52 & 52.80 \\
\hline 50 & 30 & 2 & 5 & 500.4 & 1212.0 & 103.20 & 60.00 \\
\hline 50 & 30 & 2 & 8 & 507.6 & 1236.0 & 100.80 & 64.80 \\
\hline 50 & 30 & 5 & 2 & 516.0 & 1251.6 & 100.08 & 68.64 \\
\hline 50 & 30 & 5 & 5 & 526.8 & 1272.0 & 98.400 & 75.60 \\
\hline
\end{tabular}




\section{Continued}

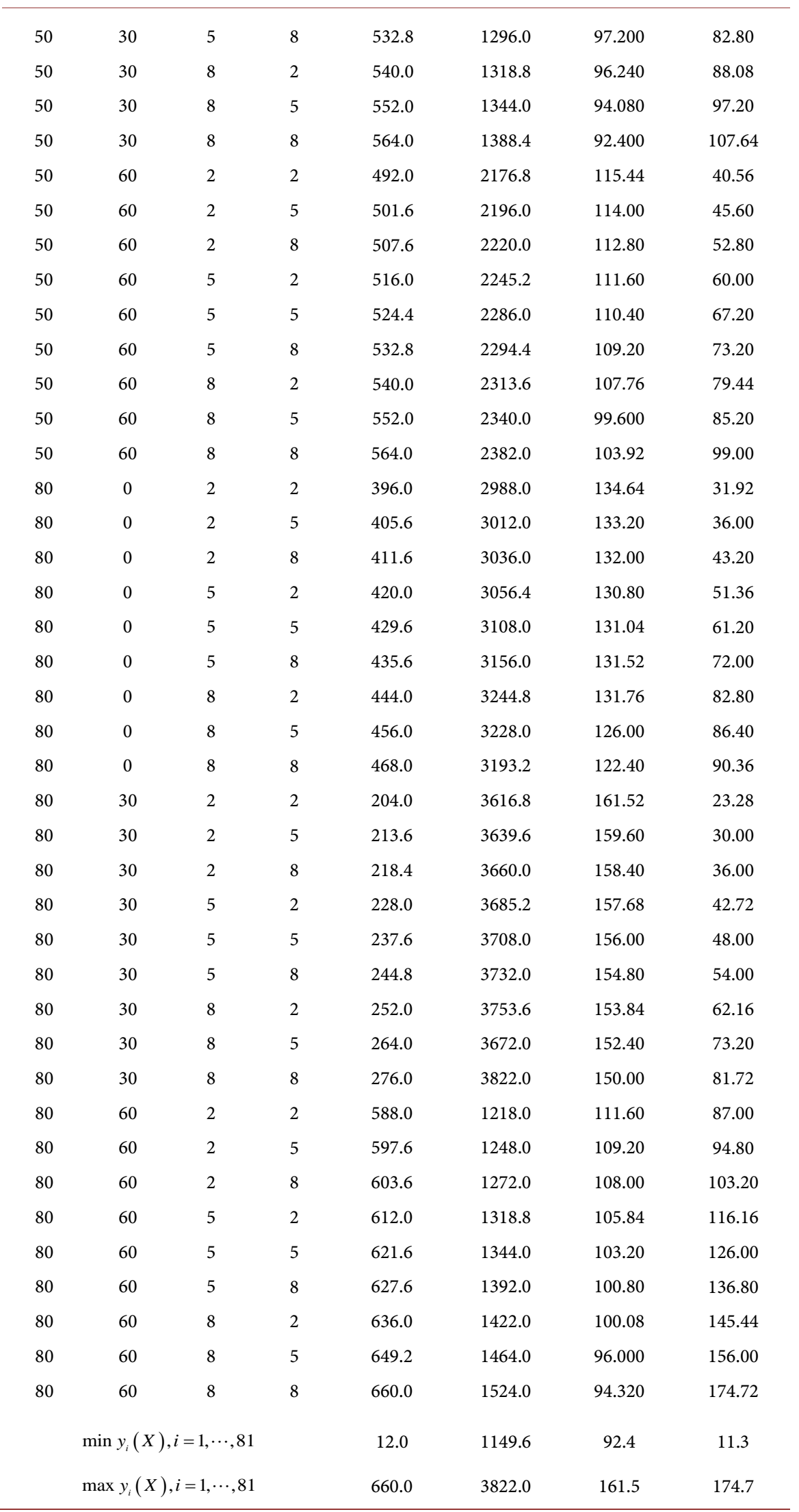


In the made decision, assessment size on the first and third characteristic it is desirable (criterion), to receive as above ( $\max$ ), on second and fourth as low as possible is possible ( $\mathrm{min}$ ). In the pilot studies the rate of an increase of parame-

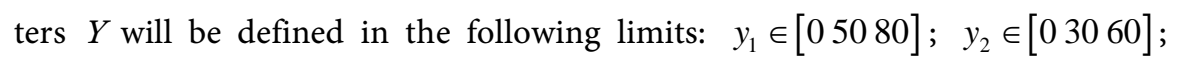
$y_{3}, y_{4} \in[258]$.

\section{It is required.}

1) To construct mathematical model of structure of the studied material in the form of a vector problem of mathematical programming.

2) To carry out model operation: first, on the basis of the constructed mathematical model, secondly, on the basis of methods of solution of a vector problem of non-linear programming at equivalent criteria, and, thirdly, the software developed for these purposes in the MATLAB system.

3) To make an optimal solution: The choice of optimum composition (structure) of material according to its functional characteristics taking into account their equivalence.

4) To choose the optimum composition of structure of material according to its functional characteristics taking into account a priority of the third criterion. The size of the third criterion $h_{3}\left(X^{q}\right)=138.2$ is received at model operation of technical system.

Stage 2. Construction of a mathematical model of the structure of material.

(Methodology of model operation of the choice of optimum structure of material in the conditions of certainty and uncertainty). It is carried out by the mathematician-the programmer. At a stage of the choice of priority criterion and its size it is carried out by the designer of material.

\subsection{Construction in the conditions of a certainty.}

The step 1 is not carried out as characteristics of material in the conditions of certainty are not set.

\subsection{Construction of a mathematical model of the structure of material.}

Construction in the conditions of indeterminacy consists in use of qualitative, quantitative descriptions of material by the principle "input-output" to Table 3. Using methods of the regression analysis, input data of Table 3:

$H(Y)=\left\{h_{1}(Y), h_{2}(Y), h_{3}(Y), h_{4}(Y)\right\}$ will be transformed to the functional type of $F(Y)=\left\{f_{1}(Y), f_{2}(Y), f_{3}(Y), f_{4}(Y)\right\}$.

Numerical values of results of the regression analysis are given below in model (112)-(117).

For creation of mathematical model of material we use results of the regression analysis. We consider the turned-out functions

$f_{1}(Y), f_{2}(Y), f_{3}(Y), f_{4}(Y)$ as criterion of a vector problem. They determine the purposefulness of characteristics of material. A set of criteria $K=4$ include two criteria of $f_{1}(Y), f_{3}(Y) \rightarrow \max$ and two $f_{2}(Y), f_{4}(Y) \rightarrow \min$. As a result model of functioning of material is represented by the vector problem of mathematical programming: 


$$
\begin{aligned}
& \text { opt } F(Y)=\{\max F_{1}(Y)=\left\{\max f_{1}(Y) \equiv 323.8-1.875 y_{1}-2.911 y_{2}+8.939 y_{3}\right. \\
&+ 10.94 y_{4}+0.0673 y_{1} y_{2}-0.0431 y_{1} y_{3}-0.1176 y_{1} y_{4}+0.0516 y_{2} y_{3} \\
&- 0.0723 y_{2} y_{4}+0.0021 y_{3} y_{4}+0.0099 y_{1}^{2}+0.0081 y_{2}^{2}-0.169 y_{3}^{2} \\
&- 0.349 y_{4}^{2}, \\
& \max f_{3}(Y) \equiv 95.71+0.6598 y_{1}+0.4493 y_{2}-0.3094 y_{3}-1.833 y_{4}-0.091 y_{1} y_{2} \\
&- 0.0057 y_{1} y_{3}+0.0134 y_{1} y_{4}-0.0119 y_{2} y_{3}+0.111 y_{2} y_{4} \\
&-\left.0.0003 y_{3} y_{4}-0.0002 y_{1}^{2}-0.0002 y_{2}^{2}+0.0233 y_{3}^{2}+0.086 y_{4}^{2}\right\}, \\
& \min F_{2}(Y)=\left\{\min f_{2}(Y) \equiv 954.8+23.9 y_{1}+30.86 y_{2}-25.85 y_{3}-45 y_{4}\right. \\
&-0.64 y_{1} y_{2}+0.3919 y_{1} y_{3}+0.6227 y_{1} y_{4}-0.1069 y_{2} y_{3} \\
&+0.2722 y_{2} y_{4}-0.0078 y_{3} y_{4}+0.0332 y_{1}^{2}+0.0304 y_{2}^{2} \\
&+2.67 y_{3}^{2}+2.205 y_{4}^{2}, \\
& 21.0-0.0081 y_{1}-0.7 y_{2}-0.3605 y_{3}+0.9769 y_{4}+0.0115 y_{1} y_{2} \\
&+0.059 y_{1} y_{3}-0.0001 y_{1} y_{4}+0.0363 y_{2} y_{3}+0.0002 y_{2} y_{4} \\
&\left.\left.+0.0003 y_{3} y_{4}-0.015 y_{1}^{2}+0.0025 y_{2}^{2}-0.042 y_{3}^{2}-0.0216 y_{4}^{2}\right\}\right\} \\
& \min f_{4}(Y) \quad \text { at } \operatorname{restrictions:} y_{1}+y_{2}+y_{3}+y_{4}=100 . \\
& 21 \leq y_{1} \leq 79,5 \leq y_{2} \leq 59,2.1 \leq y_{3} \leq 9,2.2 \leq y_{4} \leq 7 .
\end{aligned}
$$

The vector problem of mathematical programming (112)-(117) represents the model of optimal decision making, i.e. the choice of the optimal structure of the material in conditions of certainty and uncertainty in the total.

Stage 3. The solution of a vector problem of mathematical programming-material model (Algorithm 1 . The solution of a vector task with equivalent criteria).

The solution of a vector problem (112)-(117) with equivalent criteria can be represented as a sequence of steps.

Step 1. Decides problem (112)-(117) by each criterion separately, at the same time the function fmincon (...) of the Matlab system is used, the appeal to the function fmincon (...) is considered in [16]. As a result of calculation for each criterion we receive optimum points: $X_{k}^{*}$ and $f_{k}^{*}=f_{k}\left(X_{k}^{*}\right), \quad k=\overline{1, K} \quad K=4$ sizes of criteria in this point, i.e. the best decision on each criterion:

$$
X_{1}^{*}=\left\{x_{1}=46.57, x_{2}=42.23, x_{3}=8, x_{4}=2.2\right\}, f_{1}^{*}=f_{1}\left(X_{1}^{*}\right)=387.99 \text {. }
$$

The result the solution of a problem of non-linear programming (112), (116)-(117) in three-dimensional frames of $x_{1}, x_{3}$ and $f_{1}(X)$ is presented on Figure 19.

$$
X_{2}^{*}=\left\{x_{1}=50.5, x_{2}=45.2, x_{3}=2.1, x_{4}=2.2\right\}, f_{2}^{*}=f_{2}\left(X_{2}^{*}\right)=2215.91 .
$$

The result the solution of a problem of non-linear programming (114), (116)-(117) in three-dimensional frames of $x_{1}, X_{3}$ and $f_{2}(X)$ is presented on Figure 20 .

$$
X_{3}^{*}=\left\{x_{1}=79, x_{2}=11.9, x_{3}=2.1, x_{4}=7\right\}, f_{3}^{*}=f_{3}\left(X_{3}^{*}\right)=150.24 ;
$$




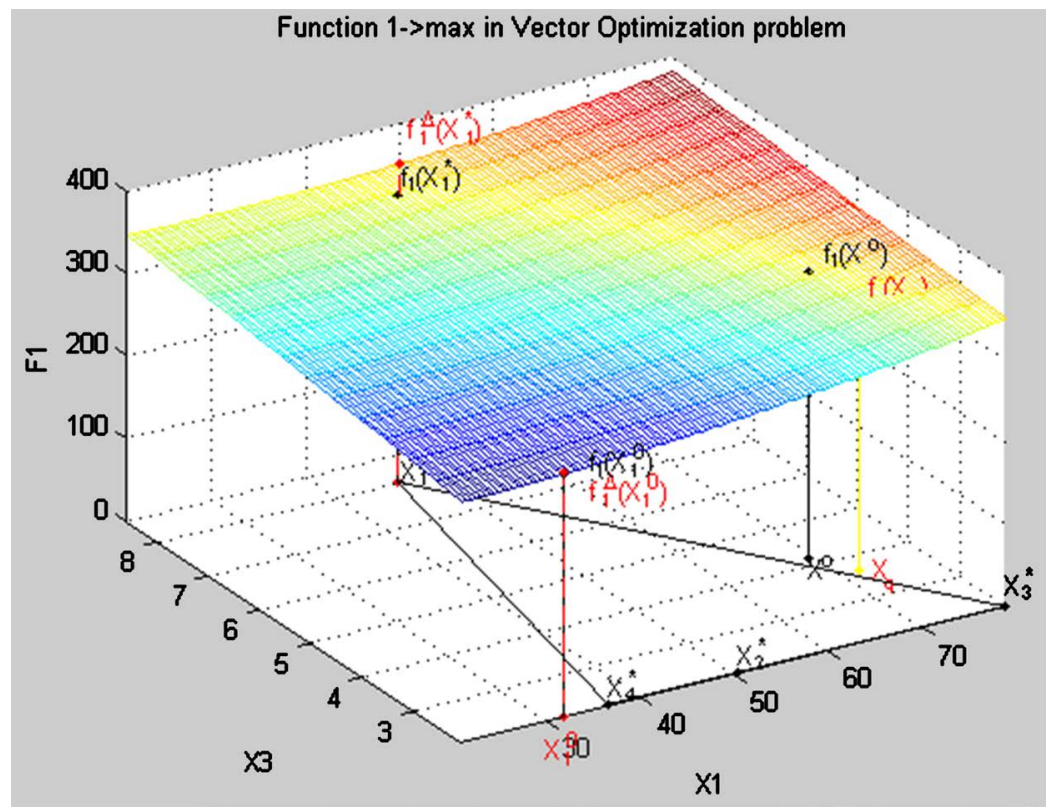

Figure 19. Result the solution of the problem (112), (116)-(117) in the three-dimensional coordinate system $\left\{X_{1}, X_{3} n f_{1}(X)\right\}$.

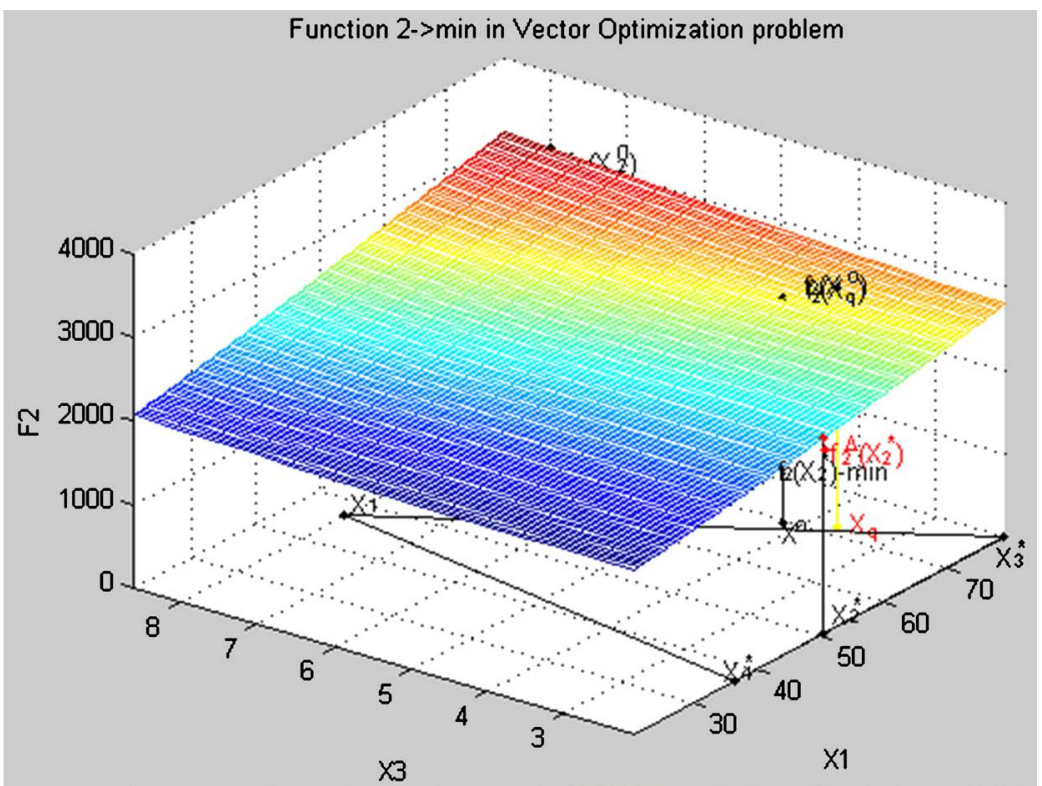

Figure 20. Result the solution of the problem (114), (116)-(117) in the three-dimensional coordinate system $\left\{x_{1}, X_{3} \mathrm{n} f_{2}(X)\right\}$.

The result the solution of a problem of non-linear programming (114), (116)-(117) in three-dimensional frames of $x_{1}, x_{3}$ and $f_{3}(X)$ is presented on Figure 21.

$$
X_{4}^{*}=\left\{x_{1}=36.7, x_{2}=59, x_{3}=2.1, x_{4}=2.2\right\}, f_{4}^{*}=f_{4}\left(X_{4}^{*}\right)=30.71 .
$$

The result the solution of a problem of non-linear programming (115), (116)-(117) in three-dimensional frames of $x_{1}, x_{3}$ and $f_{4}(X)$ is presented on Figure 22. 


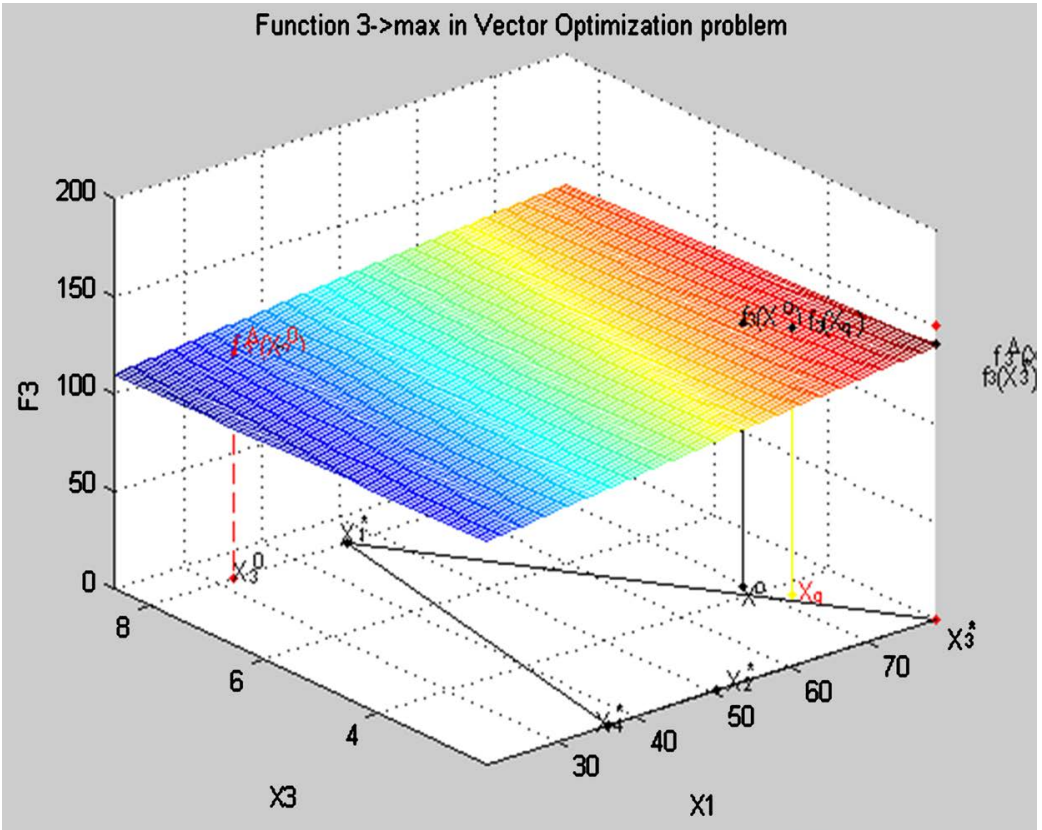

Figure 21. Result the solution of the problem (113), (116)-(117) in the three-dimensional coordinate system $\left\{X_{1}, X_{3} \Lambda f_{3}(X)\right\}$.

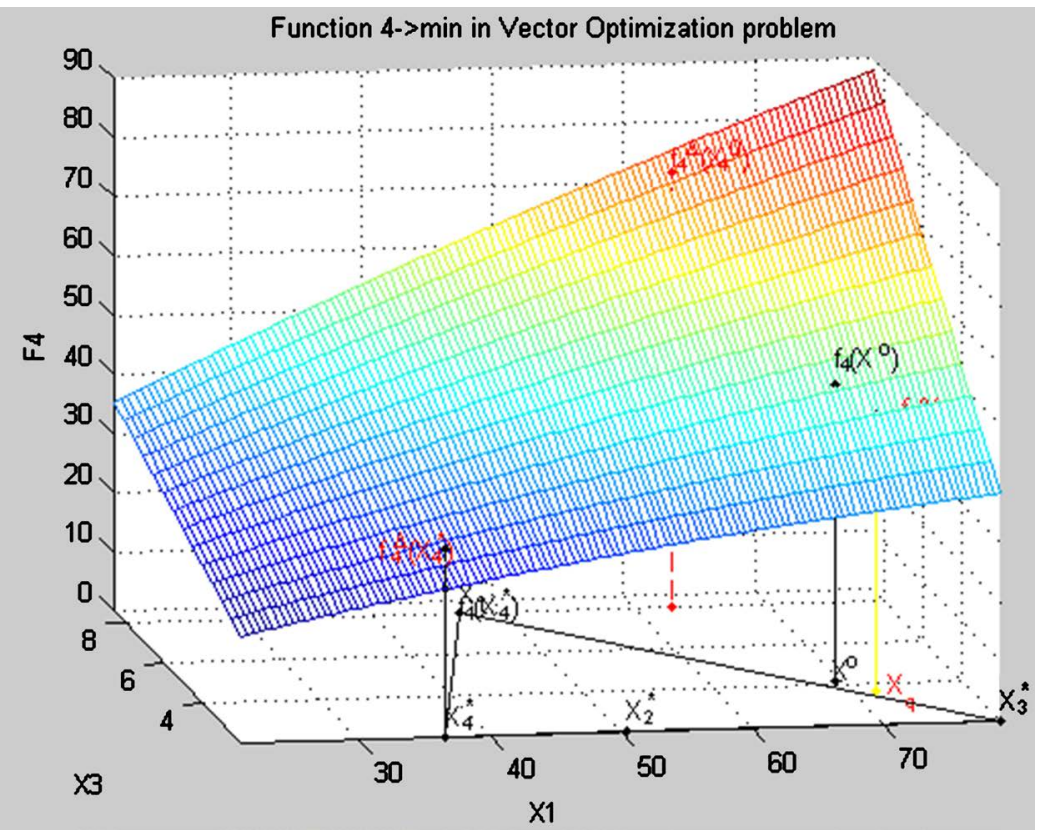

Figure 22. Result the solution of the problem (115), (116)-(117) in the three-dimensional coordinate system $\left\{X_{1}, X_{3} \Lambda f_{4}(X)\right\}$.

The location of the optimum points $X_{1}^{*}, X_{2}^{*}, X_{3}^{*}, X_{4}^{*}$ in the region of the constraints (116)-(117) in the coordinates $\left\{X_{1}, X_{3}\right\}$ is shown in Figure 23. The set of points of $S^{o}$ lying in the domain of restrictions between the points $X_{1}^{*}, X_{2}^{*}, X_{3}^{*}, X_{4}^{*}$ represent a set of Pareto optimal points.

Step 2. The worst unchangeable part of each criterion is defined (anti-optimum) (A superscript zero): 


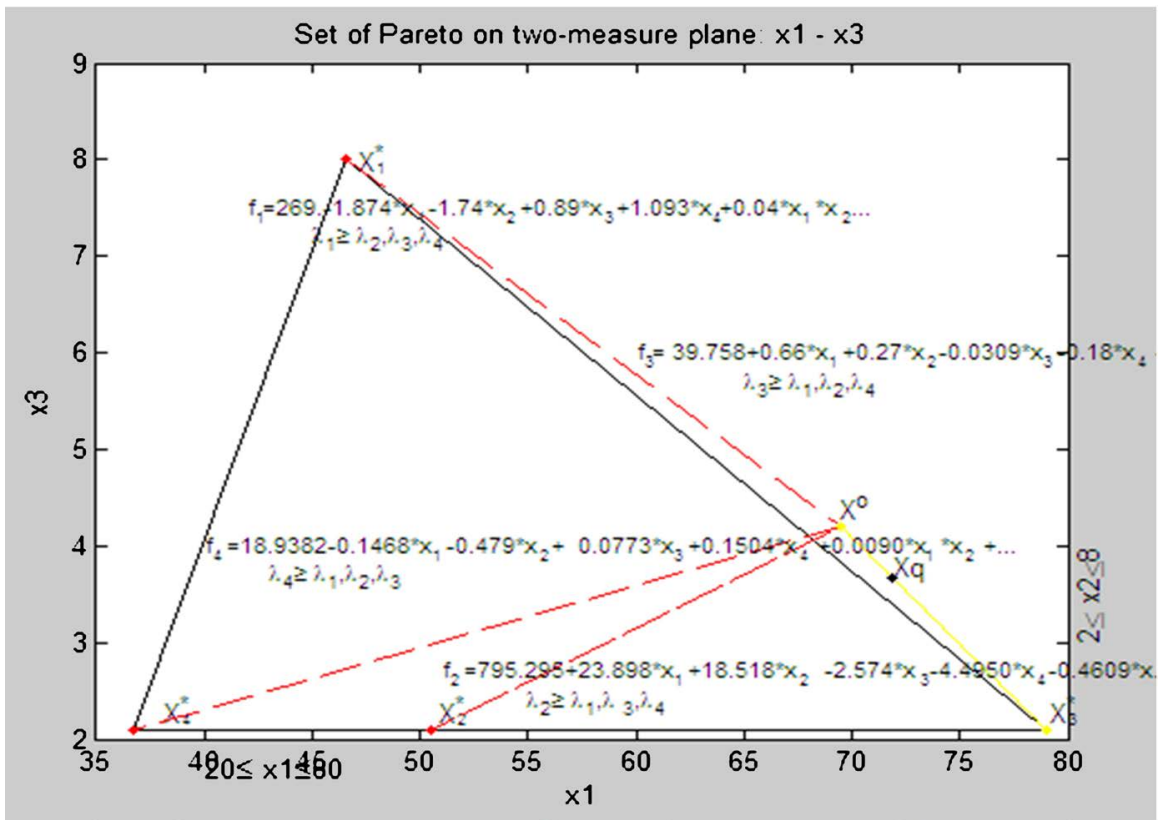

Figure 23. The set of admissible points and Pareto optimal $S^{\circ} \subset S$ in the coordinate system $\left\{X_{1}, X_{3}\right\}$.

$$
\begin{gathered}
X_{1}^{0}=\left\{x_{1}=31.9, x_{2}=59, x_{3}=2.1, x_{4}=7\right\}, f_{1}^{0}=f_{1}\left(X_{1}^{0}\right)=296.6 ; \\
X_{2}^{0}=\left\{x_{1}=79.0, x_{2}=6.0, x_{3}=8.0, x_{4}=7\right\}, f_{2}^{0}=f_{2}\left(X_{2}^{0}\right)=-3903.1 ; \\
X_{3}^{0}=\left\{x_{1}=31.9, x_{2}=57.43, x_{3}=8.0, x_{4}=2.666\right\}, f_{3}^{0}=\left(X_{3}^{0}\right)=114.87 ; \\
X_{4}^{0}=\left\{x_{1}=62.71, x_{2}=22.89, x_{3}=8.0, x_{4}=6.399\right\}, f_{4}^{0}=f_{4}\left(X_{4}^{0}\right)=73.63
\end{gathered}
$$

The obtained points of the anti-optimum $X_{1}^{0}, X_{2}^{0}, X_{3}^{0}, X_{4}^{0}$. are shown in Figure 19, ..., Figure 22 respectively.

Step 3. Systems analysis of a set of points that are Pareto-optimal is performed, (i.e. the analysis by each criterion). In points of an optimum of $X^{*}=\left\{X_{1}^{*}, X_{2}^{*}, X_{3}^{*}, X_{4}^{*}\right\}$ sizes of target functions of $F\left(X^{*}\right)=\left\|f_{q}\left(X_{k}^{*}\right)\right\|_{q=1, \overline{1}, \overline{1}}^{k=\bar{K}}$, a vector of $D=\left(d_{1} d_{2} d_{3} d_{4}\right)^{\mathrm{T}}$ of deviations are determined by each criterion on an admissible set of $S: \quad d_{k}=f_{k}^{*}-f_{k}^{0}, k=\overline{1,4}$, and matrix of the relative estimates of

$$
\begin{gathered}
\lambda\left(X^{*}\right)=\left\|\lambda_{q}\left(X_{k}^{*}\right)\right\|_{q=1, \overline{1, K}}^{k=\overline{1, K}}, \text { where } \lambda_{k}(X)=\left(f_{k}^{*}-f_{k}^{0}\right) / d_{k} . \\
F\left(X^{*}\right)=\left\|\begin{array}{cccc}
388.01 & 2401.2 & 117.5 & 68.5 \\
353.0 & 2215.9 & 124.9 & 37.5 \\
264.2 & 3445.7 & 150.2 & 34.0 \\
330.1 & 2408.9 & 122.7 & 30.7
\end{array}\right\|, \quad d_{k}=\left\|\begin{array}{c}
91.4 \\
-1725.0 \\
35.36 \\
-42.9
\end{array}\right\|, \\
\lambda\left(X^{*}\right)=\left\|\begin{array}{cccc}
1.0000 & 0.8926 & 0.0742 & 0.1197 \\
0.6171 & 1.0000 & 0.2832 & 0.8429 \\
-0.3539 & 0.2872 & 1.0000 & 0.9239 \\
0.3669 & 0.8881 & 0.2208 & 1.0000
\end{array}\right\| .
\end{gathered}
$$


The analysis of sizes of criteria in the relative estimates shows that at the points of the optimum $X^{*}=\left\{X_{1}^{*}, X_{2}^{*}, X_{3}^{*}, X_{4}^{*}\right\}$ (on diagonal) the relative assessment is equal to unit. Other criteria there is much less unit. It is required to find such point (parameters) at which the relative estimates are closest to unit. The solution of this problem is directed to the solution of $\lambda$-problem-step 4 .

Step 4. Creation of the $\lambda$-problem is carried out in two stages: originally the maximine problem of optimization with the normalized criteria is under construction:

$$
\lambda^{0}=\max _{X \in S} \min _{k \in K} \lambda_{k}(X), G(X) \leq 0, X \geq 0,
$$

which at the second stage will be transformed to a reference problem of mathematical programming $(\lambda$-problem):

$$
\begin{gathered}
\lambda^{o}=\max \lambda, \\
\text { at restrictions } \lambda-\frac{f_{1}(Y)-f_{1}^{0}}{f_{1}^{*}-f_{1}^{0}} \leq 0, \\
\lambda-\frac{f_{3}(Y)-f_{3}^{0}}{f_{3}^{*}-f_{3}^{0}} \leq 0, \\
\lambda-\frac{f_{2}(Y)-f_{2}^{0}}{f_{2}^{*}-f_{2}^{0}} \leq 0, \\
\lambda-\frac{f_{4}(Y)-f_{4}^{0}}{f_{4}^{*}-f_{4}^{0}} \leq 0, \\
x_{1}+x_{2}+x_{3}+x_{4}=100, \\
21 \leq x_{1} \leq 79,5 \leq x_{2} \leq 59,2.1 \leq x_{3} \leq 9,2.2 \leq x_{4} \leq 7 .
\end{gathered}
$$

where the vector of unknowns has dimension of $N+1: \boldsymbol{X}=\left\{x_{1}, \cdots, x_{N}, \lambda\right\}$, $N=4$.

Step 5. Solution of the $\lambda$-problem. For this purpose we use the function fmin$\operatorname{con}(\ldots)[16]:[\mathrm{Xo}][\mathrm{Lo}]=$ fmincon $\left({ }^{Z} Z_{-}\right.$Mater_4Krit_L', X0, Ao, bo, Aeq, beq, lbo, ubo, 'Z_Mater_LConst').

As a result of the solution of VPMP (112)-(1117) at equivalent criteria and $\lambda$-problem corresponding to it (121)-(123) received:

$$
\boldsymbol{X}^{o}=\left\{X^{o}, \lambda^{o}\right\}=\left\{X^{o}=\left\{x_{1}=69.5, x_{2}=24.1, x_{3}=4.144, x_{4}=2.2\right\}, \lambda^{o}=0.546\right\}-
$$

an optimum point-design data of material, $\boldsymbol{X}^{\text {o }}$, we will present in Figure 24, Figure 25;

$$
\begin{gathered}
f_{k}\left(X^{o}\right), k=1, \overline{1, K}_{\pi} \text {-sizes of criteria (characteristics of material): } \\
F\left(X^{o}\right)=\left\{f_{1}\left(X^{o}\right)=346.5, f_{2}\left(X^{o}\right)=2693.0, f_{3}\left(X^{o}\right)=134.2, f_{4}\left(X^{o}\right)=50.2\right\} \\
\lambda_{k}\left(X^{o}\right), k=\overline{1, K} \text {-sizes of the relative estimates } \\
\lambda\left(X^{o}\right)=\left\{\lambda_{1}\left(X^{o}\right)=0.546, \lambda_{2}\left(X^{o}\right)=0.72, \lambda_{3}\left(X^{o}\right)=0.546, \lambda_{4}\left(X^{o}\right)=0.546\right\} \\
\lambda^{o}=0.546 \text { is the maximum lower level among all relative estimates measured }
\end{gathered}
$$




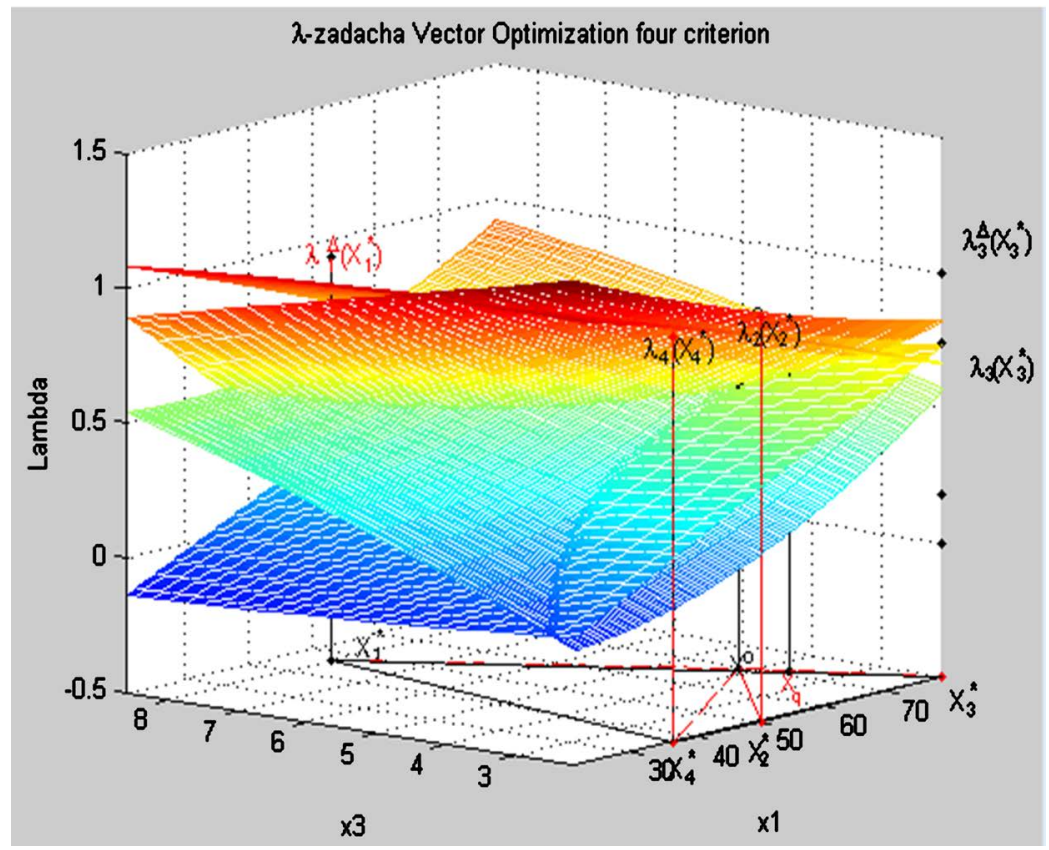

Figure 24. Geometric interpretation of the solution of the $\lambda$-problem coordinate $x_{1}, x_{3}$ and $\lambda$.

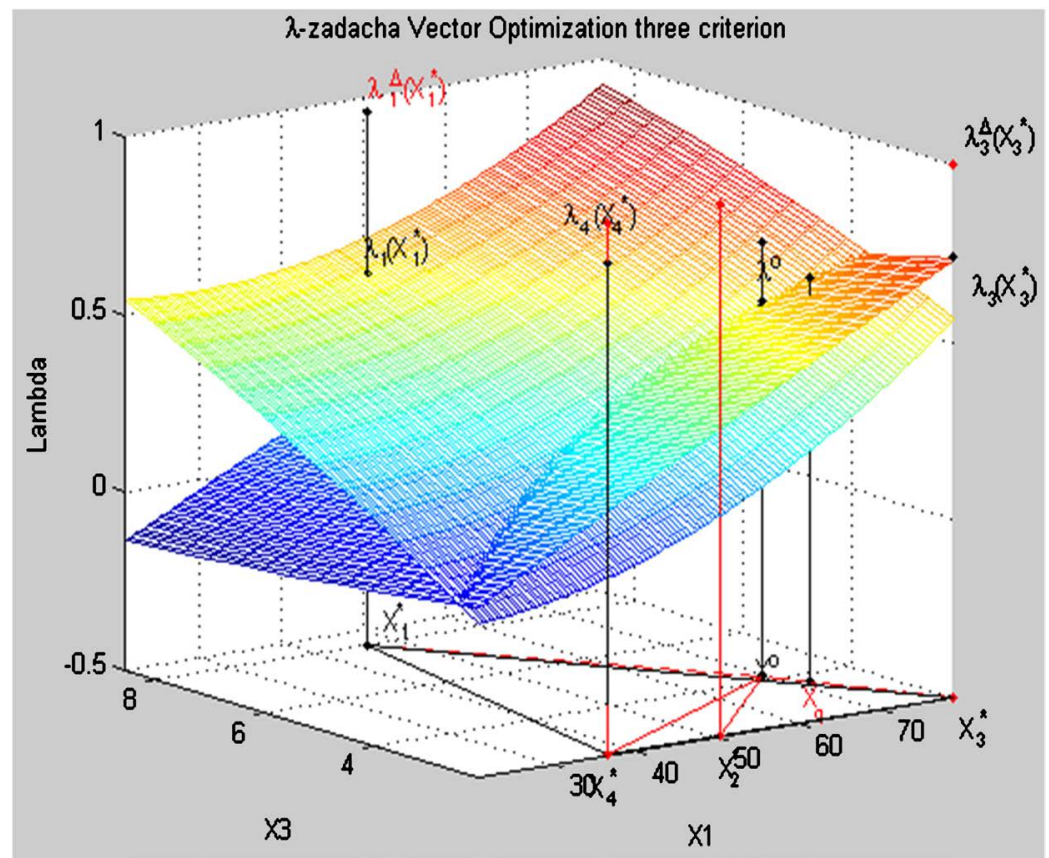

Figure 25. Solution of the $\lambda$-problem: 1,3 criteria in the coordinates $x_{1}, x_{3}$ and $\lambda$.

in the relative units: $\lambda^{o}=\min \left(\lambda_{1}\left(X^{o}\right), \lambda_{2}\left(X^{o}\right), \lambda_{3}\left(X^{o}\right), \lambda_{4}\left(X^{o}\right)\right), \lambda^{o}$-also call the guaranteed result in the relative units, i.e. $\lambda_{k}\left(X^{0}\right)$ and according to the characteristic of the material $f_{k}\left(X^{o}\right)$ it is impossible to improve, without worsening at the same time other characteristics.

Let's notice that according to the theorem 2 [4], in $X^{o}$ point criteria 1, 3, 4 are contradictory. This contradiction is defined by equality of 
$\lambda^{o}=\min \left(\lambda_{1}\left(X^{o}\right), \lambda_{2}\left(X^{o}\right), \lambda_{3}\left(X^{o}\right), \lambda_{4}\left(X^{o}\right)\right), \quad \lambda^{o}=0.546$, and other criteria inequality $\left\{\lambda_{k}\left(X^{o}\right)\right\}>\lambda^{\circ}$.

Stage 4. Creation of geometrical interpretation of results of the decision in a three-dimensional coordinate system.

In an admissible point set of $S$ formed by restrictions (116)-(117), optimum points $X_{1}^{*}, X_{2}^{*}, X_{3}^{*}, X_{4}^{*}$, united in a contour, present a point set, Pareto optimal, to $S^{o} \subset \boldsymbol{S}$. Coordinates of these points and also characteristics of material in the relative units: $\lambda_{1}\left(X_{1}^{*}\right), \lambda_{2}\left(X_{2}^{*}\right), \lambda_{3}\left(X_{3}^{*}\right), \lambda_{4}\left(X_{4}^{*}\right)$ are shown in Figure 24 in three measuring space: $x_{1}, X_{3}$ and $\lambda$, where the third axis of $\lambda$-the relative assessment.

Let's carry out the analysis of results of the solution of a vector problem (the analysis of the choice of an optimal solution at equivalent criteria-characteristics), using geometrical interpretation. For this purpose in Figure 5, Figure 6 we will connect points: $X_{1}^{*}, X_{2}^{*}, X_{3}^{*}, X_{4}^{*}$ with a point of $X^{o}$ which conditionally represents the centre of a Pareto set. As a result conditionally received four subsets of points of $X \in S_{q}^{o} \subset S^{o} \subset S, q=\overline{1,4}$. The subset of $S_{1}^{o} \subset S^{o} \subset S$ is characterized by the fact that the relative assessment of $\lambda_{1} \geq \lambda_{2}, \lambda_{3}, \lambda_{4}$, i.e. in the field of $S_{1}^{0}$ first criterion has a priority over the others (definition of a priority see [12]). Similar to $S_{2}^{o}, S_{3}^{o}, S_{4}^{o}$-subsets of points where the second, third, fourth criterion has a priority over the others respectively. A point set, Pareto optimal we will designate

$$
S^{o}=S_{1}^{o} \cup S_{2}^{o} \cup S_{3}^{o} \cup S_{4}^{o} .
$$

Coordinates of all received points and the relative estimates are presented in two dimensional space in Figure 23. These coordinates are shown in three measuring space $\left\{x_{1}, x_{3}, \lambda\right\}$ on the side of $\lambda^{o}$. Restrictions of a point set, Pareto optimal, in Figure 24, Figure 25 it is lowered to -0.5 (that restrictions are visible). This information is also a basis for a further research of structure of a Pareto set (a set of options of structures of material).

Stage 5. Decision-making in the structure of material model at the set priority of criterion.

\section{(Algorithm 2. The solution of a vector task with a criterion priority)}

The person making decisions, as a rule, is the designer of material.

Step 1. The solution of a vector problem with equivalent criteria. Results of the decision are presented in Section 4.3. If results of the solution of a vector problem with equivalent criteria do not satisfy the person making the decision, then the choice of an optimal solution is carried out from any subset of points of $\boldsymbol{S}_{1}^{o}, \boldsymbol{S}_{2}^{o}, \boldsymbol{S}_{3}^{o}, \boldsymbol{S}_{4}^{o}$.

Step 2. Choice of priority criterion of $q \in \boldsymbol{K}$.

From the theory (the Theorem 2 [4]) it is known that in an optimum point of $X^{o}$ there are always two most contradictory criteria: $q \in \boldsymbol{K}$ and $v \in \boldsymbol{K}$ for which in the relative units precise equality is carried out:

$$
\lambda^{o}=\lambda_{q}\left(X^{o}\right)=\lambda_{p}\left(X^{o}\right), q, p \in K, X \in S,
$$


and for the others it is carried out inequalities: $\lambda^{o} \leq \lambda_{k}\left(X^{o}\right), \forall k \in K, q \neq p \neq k$.

For the choice of priority criterion on the display the message about results of the solution of $\lambda$-problem in physical and relative units is given:

Criteria (128) in $X^{o}$ optimum point:

$$
F\left(X^{o}\right)=\left\{f_{1}\left(X^{o}\right)=346.5, f_{2}\left(X^{o}\right)=2693.0, f_{3}\left(X^{o}\right)=134.2, f_{4}\left(X^{o}\right)=50.2\right\} \text {. }
$$

The relative estimates (129) in $X^{0}$ :

$$
\lambda\left(X^{o}\right)=\left\{\lambda_{1}\left(X^{o}\right)=0.546, \lambda_{2}\left(X^{o}\right)=0.7235, \lambda_{3}\left(X^{o}\right)=0.546, \lambda_{4}\left(X^{o}\right)=0.546\right\} .
$$

From here it is visible (conclusion), in model of material (112)-(117) and the corresponding $\lambda$-problem (121)-(123) such criteria are the first, third and fourth:

$$
\lambda^{o}=\lambda_{1}\left(X^{o}\right), \lambda_{3}\left(X^{o}\right), \lambda_{4}\left(X^{o}\right)=0.546,
$$

Let's show the first and third in Figure 7 on the basis of which we will conduct their further research.

As a rule, from a pair of conflicting criteria, a criterion chosen by the decision maker would be improved. Such criterion is called "priority criterion", we will designate it $q=3 \in \boldsymbol{K}$. This criterion is investigated in interaction with the first criterion of $q=1 \in \boldsymbol{K}$. On the display the message is given:

$q=$ input ('Enter priority criterion (number) of $q=$ ') -Entered: $q=3$.

Step 3. Numerical limits of change of size of a priority of criterion of $q=3 \in \boldsymbol{K}$ are defined. For priority criterion of $q=3 \in \boldsymbol{K}$ changes of numerical limits in physical units upon transition from $X^{o}$ optimum point to the point of $X_{q}^{*}$ received on the first step at equivalent criteria are defined. $q=3$ given about criterion are given for the screen:

$$
f_{q}\left(X^{\circ}\right)=134.183 \leq f_{q}(X) \leq 150.238=f_{q}\left(X_{q}^{*}\right), q \in \boldsymbol{K} .
$$

In the relative units the criterion of $q=3$ changes in the following limits:

$$
\lambda_{q}\left(X^{o}\right)=0.546 \leq \lambda_{q}(X) \leq 1=\lambda_{q}\left(X_{q}^{*}\right), q=3 \in \boldsymbol{K} .
$$

These data it is analyzed.

Step 4. Choice of size of priority criterion of $q \in \boldsymbol{K}$. (Decision-making).

On the message: "Enter the size of priority criterion $f_{q}=$ "-we enter, the size of the characteristic defining structure of material: $f_{q}=138.2$.

\section{Step 5. The relative assessment is calculated.}

For the chosen size of priority criterion $f_{q}=1500$ the relative assessment is calculated:

$$
\lambda_{q}=\frac{f_{q}-f_{q}^{0}}{f_{q}^{*}-f_{q}^{0}}=\frac{80-55.73}{87.71-55.73}=0.6596,
$$

which upon transition from $X^{o}$ point to $X_{3}^{*}$ lies in limits:

$$
\lambda_{q}\left(X^{o}\right)=0.546 \leq \lambda_{q}(X) \leq 0.6596 \leq \lambda_{q}\left(X_{q}^{*}\right), q=3 \in \boldsymbol{K} .
$$

Step 6. Let's calculate coefficient of the linear approximation. 
Assuming the linear nature of change of criterion of $f_{q}(X)$ in (127) and according to the relative assessment of $\lambda_{q}$, using reference methods of the linear approximation, we will calculate a constant of proportionality between $\lambda_{q}\left(X^{o}\right), \lambda_{q}$ which we will call $\rho$ :

$$
\rho=\frac{\lambda_{q}-\lambda_{q}\left(X^{o}\right)}{\lambda_{q}\left(X_{q}^{*}\right)-\lambda_{q}\left(X^{o}\right)}=\frac{0.8589-0.5534}{1-0.5534}=0.2502, q=3 \in \boldsymbol{K} .
$$

Step 7. Let's calculate coordinates of a priority of criteria with dimension of $f_{q}$ Assuming the linear nature of change of a vector of $X^{q}=\left\{x_{1} x_{3}\right\}, q=3$ we will determine point coordinates with dimension of $f_{q}=138.2$, the relative assessment (128):

$$
\begin{aligned}
x_{\lambda=0.6596}^{q=3}=\left\{x_{1}=X^{o}(1)+\rho\left(X_{q}^{*}(1)-X^{o}(1)\right), x_{2}=X^{o}(2)+\rho\left(X_{q}^{*}(2)-X^{o}(2)\right),\right. \\
\left.x_{3}=X^{o}(3)+\rho\left(X_{q}^{*}(3)-X^{o}(3)\right), x_{4}=X^{o}(4)+\rho\left(X_{q}^{*}(4)-X^{o}(4)\right)\right\},
\end{aligned}
$$

where $X^{o}=\left\{x_{1}=69.5, x_{2}=24.1, x_{3}=4.144, x_{4}=2.2\right\}$,

$$
X_{3}^{*}=\left\{x_{1}=79, x_{2}=11.9, x_{3}=2.1, x_{4}=7\right\} \text {. }
$$

As result of the decision (130) we will receive $X^{q}$ point with coordinates:

$$
X^{q}=\left\{x_{1}=71.877, x_{2}=21.05, x_{3}=3.673, x_{4}=3.4\right\} \text {. }
$$

Step 8. Calculation of the main indexes of a point of $X_{q}$.

For the received $X^{q}$ point, we will calculate:

all criteria in physical units $f_{k}\left(X^{q}\right)=\left\{f_{k}\left(X^{q}\right), k=\overline{1, K}\right\}$,

$$
\begin{aligned}
f\left(X^{q}\right)=\{ & f_{1}\left(X^{q}\right)=329.5, f_{2}\left(X^{q}\right)=2841.8, \\
f_{3}\left(X^{q}\right) & \left.=137.4, f_{4}\left(X^{q}\right)=46.6\right\}
\end{aligned}
$$

all relative estimates of criteria $\lambda^{q}=\left\{\lambda_{k}^{q}, k=\overline{1, K}\right\}$,

$$
\begin{aligned}
\lambda_{k}\left(X^{q}\right)=\frac{f_{k}\left(X^{q}\right)-f_{k}^{0}}{f_{k}^{*}-f_{k}^{0}}, k=\overline{1, K}_{\pi}, \\
\lambda_{k}\left(X^{q}\right)=\left\{\lambda_{1}\left(X^{q}\right)=0.1929, \lambda_{2}\left(X^{q}\right)=0.5328,\right. \\
\left.\lambda_{3}\left(X^{q}\right)=0.7242, \lambda_{4}\left(X^{q}\right)=0.7117\right\} .
\end{aligned}
$$

Analysis of results. The calculated size of criterion $f_{q}\left(X_{t}^{o}\right), q \in \boldsymbol{K}$ is usually not equal to the set $f_{q}$. The error of the choice of $\Delta f_{q}=\left|f_{q}\left(X_{t}^{o}\right)-f_{q}\right|=|137.4-138.2|=0.8$ is defined by an error of linear approximation, $\Delta f_{q \%}=0.04 \%$.

In the course of modeling parametrical restrictions (116)-(117) can be changed, i.e. some set of optimum decisions is received. Choose a final version which in our example included from this set of optimum decisions:

parameters of material: $X^{o}=\left\{x_{1}=69.5, x_{2}=24.1, x_{3}=4.144, x_{4}=2.2\right\}$,

$$
X^{\text {summa }}=x_{1}+x_{2}+x_{3}+x_{4}=100
$$


the parameters of the material at a given priority criterion $q=3$ :

$$
\begin{gathered}
X^{q}=\left\{x_{1}=71.877, x_{2}=21.05, x_{3}=3.673, x_{4}=3.4\right\}, \\
X^{\text {summa }}=x_{1}+x_{2}+x_{3}+x_{4}=100 .
\end{gathered}
$$

\section{Conclusions}

The problem of developing mathematical methods of vector optimization and making optimal decisions based on them in a complex engineering system for a set of experimental data and functional characteristics is one of the most important tasks of system analysis and design.

The methodology of constructing a mathematical model of an engineering system under conditions of certainty and uncertainty in the form of a vector problem of mathematical programming is developed. To solve the vector problem, new methods of vector optimization based on the normalization of criteria and the principle of guaranteed results are developed. Vector optimization methods allow us to make a decision, firstly, with equivalent criteria, and secondly, with a given criterion priority. When building characteristics in the conditions of uncertainty used regression methods of data conversion. The practice of "optimal decision-making" based on the mathematical model is shown on the numerical example of solving the vector optimization problem.

These methods of processing of the expert data and vector optimization can be used at design of engineering systems of various branches: electrotechnical, aerospace, metallurgical, etc. At creation of characteristics in the conditions of uncertainty regression methods of transformation of information are used. This methodology has system character and can be used when modeling technical, economic and other systems. Author is ready to participate in the solution of vector problems of linear and nonlinear programming.

\section{Conflicts of Interest}

The author declares no conflicts of interest regarding the publication of this paper.

\section{References}

[1] Mashunin, Y.K. (1986) Methods and Models of Vector Optimization. Nauka, Moscow. [In Russian]

[2] Mashunin, Y.K. and Levitskii, V.L. (1996) Methods of Vector Optimization in Analysis and Synthesis of Engineering Systems. DVGAEU, Vladivostok. [In Russian]

[3] Mashunin, Y.K. (2000) Engineering System Modeling on the base of Vector Problem of Nonlinear Optimization. Control Applications of Optimization. Preprint of the 11th IFAC International Workshop, CAO 2000, Saint Petersburg.

[4] Mashunin, Y.K. (1999) Solving Composition and Decomposition Problems of Synthesis of Complex Engineering Systems by Vector Optimization Methods. Journal of Computer and Systems Sciences International, 52, 519-534. 
[5] Mashunin, K.Y. and Mashunin, Y.K. (2013) Simulation Engineering Systems under Uncertainty and Optimal Decision Making. Journal of Computer and Systems Sciences International. 52, 519-534. https://doi.org/10.1134/S106423071303012X

[6] Mashunin, Y.K. (2013) Control Theory. The Mathematical Apparatus of Management of the Economy. Logos, Moscow, 448 p. (In Russian)

[7] Mashunin, Y.K. and Mashunin, K.Y. (2014) Modeling of Technical Systems on the Basis of Vector Optimization (1. at Equivalent Criteria). International Journal of Engineering Sciences \& Research Technology, 3, 84-96.

[8] Mashunin, Y.K. and Mashunin, K.Y. (2014) Modeling of technical Systems on the Basis of Vector Optimization (2. with a Criterion Priority). International Journal of Engineering Sciences \& Research Technology, 3, 224-240.

[9] Mashunin, Y.K. and Mashunin, K.Y. (2015) Simulation and Optimal Decision Making the Design of Technical Systems. American Journal of Modeling and Optimization, 3, 56-67.

[10] Mashunin, Y.K. and Mashunin, K.Y. (2016) Simulation and Optimal Decision Making the Design of Technical Systems (2. The Decision with a Criterion Priority). American Journal of Modeling and Optimization, 4, 51-66.

[11] Mashunin, Y.K. (2019) Methodology of the Choice of Optimum Parameters of Technological Process on Experimental to Data. American Journal of Modeling and Optimization, 7, 20-28.

[12] Mashunin, Y.K. (2017) Vector Optimization in the System Optimal Decision Making the Design in Economic and Technical Systems. International Journal of Emerging Trends \& Technology in Computer Science, 7, 42-57.

[13] Mashunin, Y.K. (2018) Optimal Designing in the Interrelation Technical System-Materials (Theory). Mathematical Methods in Engineering and Technology. In Bolshakova, A.A., Ed., Proceedings of the International Scientific Conference: 12 tons 4/ under General, Publishing House of Polytechnical Institute, 40-46.

[14] Mashunin, Y.K. (2017) Concept of Technical Systems Optimum Designing (Mathematical and Organizational Statement). 2017 International Conference on Industrial Engineering, Applications and Manufacturing, Saint Petersburg, 16-19 May 2017, 1-5.

[15] Mashunin, Y.K. (2017) Optimum Designing of the Technical Systems Concept (Numerical Realization). 2017 International Conference on Industrial Engineering, Applications and Manufacturing, Saint Petersburg, 16-19 May 2017, 1-6.

[16] Mashunin, K.Y. and Mashunin, Y.K. (2017) Vector Optimization with Equivalent and Priority Criteria. Journal of Computer and Systems Sciences International, 56, 975-996. https://rdcu.be/bhZ8i https://doi.org/10.1134/S1064230717060119

[17] Torgashov, A.Y., Krivosheev, V.P., Mashunin, Y.K. and Holland, C.D. (2001) Calculation and Multiobjective Optimization of Static Modes of Mass Exchange Processes by the Example of Absorption in Gas Separation. Izvestiya vysshikh uchebnykh zavedenii. Neft' $i$ Gaz, No. 3, 82-86.

[18] Ketkov, Y.L., Ketkov, A.Y. and Shul'ts, M.M. (2004) MATLAB 6.x.: Numerical Programming. BKhV Peterburg, St. Petersburg, 672 p. [In Russian]

[19] Keeney, R.L. and Raiffa, H. (1976) Decisions with Multiple Objectives-Preferences and Value Tradeoffs. Wiley, New York.

[20] Johannes, J. (2010) Vector Optimization: Theory, Applications, and Extensions. Springer-Verlag, Berlin, 510 p. 
[21] Ansari, Q. and Jen-Chih, Y. (2010) Recent Developments in Vector Optimization. Springer, Heidelberg.

[22] Hirotaka, N., Yeboon, Y. and Min, Y. (2009) Sequential Approximate Multiobjective Optimization Using Computational Intelligence. Springer-Verlag, Berlin, 197 p.

[23] Shankar, R. (2007) Decision Making in the Manufacturing Environment: Using Graft Theory and Fuzzy Multiple Attribute Decision Making Methods. Springer-Verlag, Berlin.

[24] Cooke, T., Lingard, H. and Blismas, N. (2008) The Development and Evaluation of a Decision Support Tool for Health and Safety in Construction Design. Engineering, Construction and Architectural Management, 15, 336-351. https://doi.org/10.1108/09699980810886847

[25] Oluwasegun, K.M., Ojo, O.A., Ola, O.T., Birur, A., Cuddy, J. and Chan, K. (2018) Development of Artificial Neural Network Models for Predicting Weld Output Parameters in Advanced Fusion Welding of a Magnesium Alloy. American Journal of Modeling and Optimization, 6, 18-34. 